\title{
Ghana: Fifth Review Under the Three-Year Arrangement Under the Extended Credit Facility and Request for Modification of Performance Criteria-Staff Report; Staff Supplements; Press Release on the Executive Board Discussion; and Statement by the Executive Director for Ghana.
}

In the context of the fifth review under the three-year arrangement under the extended credit facility and request for modification of performance criteria, the following documents have been released and are included in this package:

- $\quad$ The staff report for the Fifth Review Under the Three-Year Arrangement Under the Extended Credit Facility and Request for Modification of Performance Criteria, prepared by a staff team of the IMF, following discussions that ended on October 25, 2011, with the officials of Ghana on economic developments and policies. Based on information available at the time of these discussions, the staff report was completed on November 29, 2011. The views expressed in the staff report are those of the staff team and do not necessarily reflect the views of the Executive Board of the IMF.

- $\quad$ A supplement containing a Joint IMF/World Bank Debt Sustainability Analysis

- A staff supplement of December 12, 2011 updating information on recent developments.

- $\quad$ A Press Release summarizing the views of the Executive Board as expressed during its December 14, 2011 discussion of the staff report that completed the request and/or review.

- $\quad$ A statement by the Executive Director for Ghana.

The documents listed below have been or will be separately released.

Letter of Intent sent to the IMF by the authorities of Ghana* Memorandum of Economic and Financial Policies by the authorities of Ghana*

Technical Memorandum of Understanding*

*Also included in Staff Report

The policy of publication of staff reports and other documents allows for the deletion of market-sensitive information.

Copies of this report are available to the public from

International Monetary Fund • Publication Services $70019^{\text {th }}$ Street, N.W. • Washington, D.C. 20431

Telephone: (202) 623-7430 • Telefax: (202) 623-7201

E-mail: publications@imf.org Internet: http://www.imf.org

\section{International Monetary Fund Washington, D.C.}


INTERNATIONAL MONETARY FUND

GHANA

\title{
Fifth Review Under the Three-Year Arrangement Under the Extended Credit Facility and Request for Modification of Performance Criteria
}

\author{
Prepared by the African Department \\ (In consultation with other departments) \\ Approved by Michael Atingi-Ego and Dominique Desruelle
}

November 29, 2011

Discussions: The review mission visited Accra during October 12-25, 2011. The staff team consisted of Ms. Daseking (head), Mr. Baldini, Ms. Verdier (all AFR), and Ms. Nkhata (SPR). Mr. Mitchell, Resident Representative, supported the team in the field, and Mr. Mojarrad, Executive Director, joined the discussion during the mission. The mission met with President John Evans Atta Mills, Finance Minister Kwabena Duffuor, Bank of Ghana Governor Kwesi Amissah-Arthur, other senior officials, members of parliament, and representatives from the private sector, academia, civil society, and Ghana's development partners.

IMF relations: Ghana joined the IMF on September 20, 1957. Ghana's current threeyear Extended Credit Facility (ECF) arrangement was approved in July 2009, and the combined third and fourth reviews were concluded in May 2011.

Exchange rate regime: Ghana maintains a floating exchange rate regime and an exchange system free of restrictions on payments and transfers for current international transactions. Ghana has accepted the obligations of Article VIII, Sections 2, 3, and 4.

Publication: The Ghanaian authorities have consented to publication of this staff report, and the attached Letter of Intent and Memorandum of Economic and Financial Policies, which review the authorities' program implementation and outline their policies for the remainder of 2011 and for 2012. 


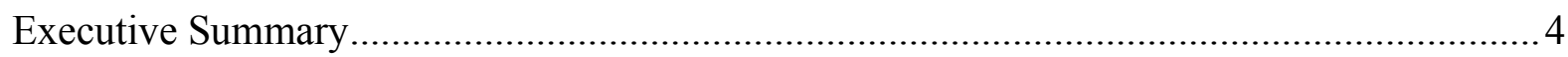

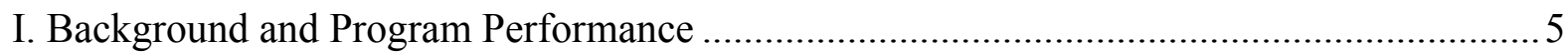

A. Strong Growth and Moderating Inflation ............................................................5

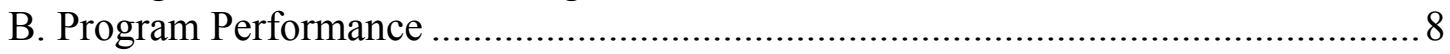

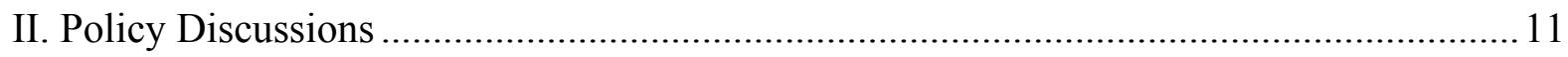

A. Setting the Stage for Scaled-Up Investment ......................................................... 11

B. Fiscal Policy: Preserving Gains to Macroeconomic Stability ................................... 14

C. Energy Policies: Responsible Management of Revenues and Prices ...................... 17

D. Monetary and Financial Policy: Safeguarding Low Inflation and

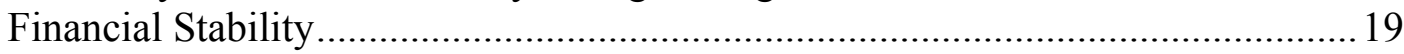

III. Program Monitoring, Modification of Performance Criteria, and Risks...........................22

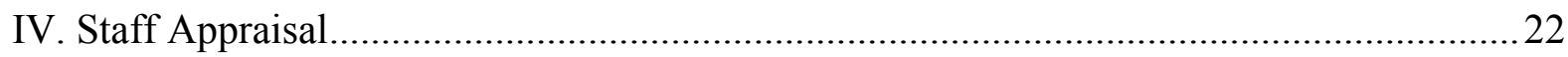

Boxes

1. The Single Spine Salary Structure and the Wage Bill …….................................. 10

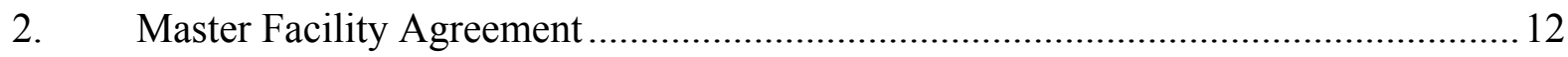

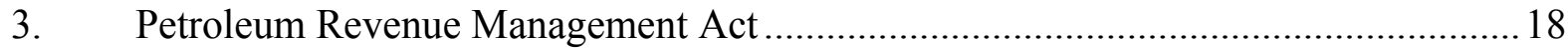

Figures

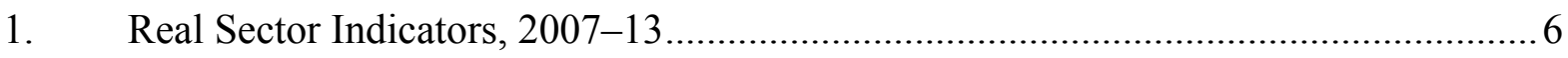

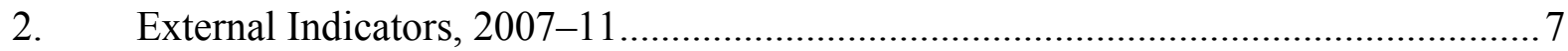

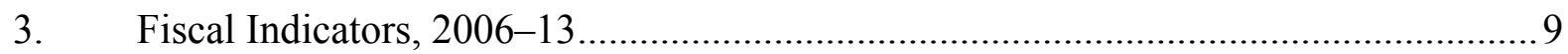

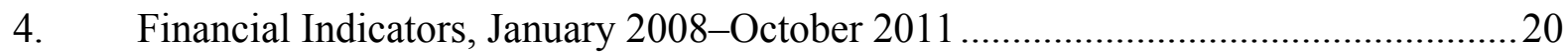

Tables

1. Selected Economic and Financial Indicators, 2008-13 ………..............................25

2A. Summary of Central Government Budgetary Operations (percent of non-oil GDP),

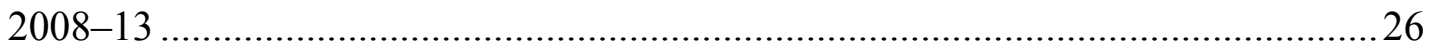

2B. Summary of Central Government Budgetary Operations (millions of GHc),

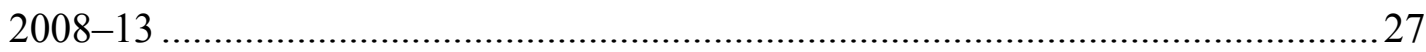

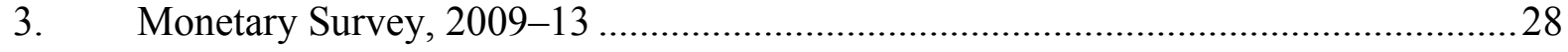

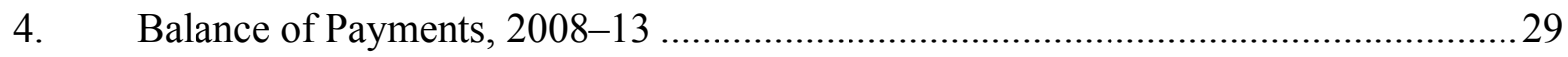

5. Financial Soundness Indicators, 2005-11 ..............................................................30

6. External Financing Requirements and Sources, 2009-16 …………........................ 31

7. Indicators of Capacity to Repay the Fund, 2011-2022 ……...................................... 32

8. Proposed Schedule of Disbursements Under the ECF Arrangement, 2009-12 ….......33 


\section{Appendix}

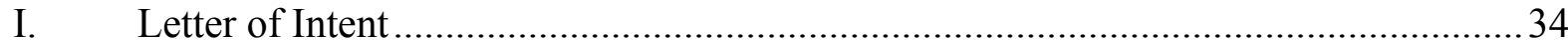

Attachment I. Memorandum of Economic and Financial Policies...........................37

Attachment II. Technical Memorandum of Understanding for the 2011-12, Extended Credit Facility Arrangement................................................................5 


\section{Executive Summary}

The stability of Ghana's economy has improved significantly since the start of the ECF in 2009.

- All end-June 2011 quantitative performance criteria were met. All but one indicative target for end-September were also met; only arrears clearance was missed by a very small margin. Structural reforms have progressed with some unevenness; six benchmarks were met, but four were missed.

- Strong growth and moderating inflation continue. Boosted by the start of oil production, economic growth is projected to reach 131/2 percent in 2011 and more than 8 percent in 2012, with average inflation expected to remain broadly stable in the upper single digits. Fiscal and external current account imbalances have been greatly reduced, and the international reserve position has strengthened.

The macroeconomic outlook is positive and the medium-term policy stance is appropriate. On the fiscal side, the government plans to scale up infrastructure investment and further boost revenues; management of the wage bill, however, has continued to be a challenge.

- The fiscal program remains appropriate. The fiscal deficit (after arrears clearance) will be about 5 percent of non-oil GDP in $2011-33 / 4$ percent on a commitment basis, compared with $93 / 4$ percent in 2010. In 2012, the targeted cash deficit will be broadly unchanged, implying a commitment-based deficit of 3 percent of non-oil GDP. The fiscal stance incorporates high-priority infrastructure investments and the full cost of migration to a new public pay structure. New revenue measures are expected to compensate for the short-term costs of migration to a uniform public pay structure.

- A revised debt sustainability analysis suggests scope for higher nonconcessional borrowing, provided fiscal targets are achieved. Staff, therefore, supports a modification of Ghana's nonconcessional borrowing limit to accommodate the authorities' borrowing plans.

- Energy reforms focus on responsible management of revenues and prices. The Petroleum Revenue Management Act provides a strong legal basis for this purpose. While energy pricing has improved, subsidies on petroleum products have reemerged in the second half of 2011.

- The Bank of Ghana has been successful in meeting its inflation target. The authorities aim at keeping inflation to 9 percent by year-end and reduce it further in 2012. The banking sector remains adequately capitalized and liquid, and reform priorities include reducing nonperforming loans and strengthening banks' risk management practices.

- Program risks arise from external and domestic sources. These include possibly weaker commodity prices and foreign investment flows, as a result of a prolonged global slowdown, and the possibility of public spending pressures ahead of the 2012 elections.

- Staff recommends completion of the fifth review. 


\section{Background ANd Program Performance}

\section{A. Strong Growth and Moderating Inflation}

1. Important stability gains since 2009. The stability of Ghana's economy has improved significantly since the beginning of the government's Fund-supported program in 2009. The then sizeable fiscal and external current account imbalances have been greatly reduced, the inflation rate has declined to single digits, and the stock of international reserves has risen to about US\$5 billion, up from only US\$2 billion at the end of 2008 (Text Table 1, Table 1, and Figures 1-2). Moreover, a recent rebasing of Ghana's national accounts has raised its national income measures by

\begin{tabular}{|c|c|c|c|c|c|}
\hline & 2008 & 2009 & 2010 & $\begin{array}{l}2011 \\
\text { Proj. }\end{array}$ & $\begin{array}{l}2012 \\
\text { Proj. }\end{array}$ \\
\hline & \multicolumn{5}{|c|}{ (Annual percentage change) } \\
\hline Real GDP & 8.4 & 4.0 & 7.7 & 13.6 & 8.5 \\
\hline Non-oil real GDP & 8.4 & 4.0 & 7.7 & 8.2 & 7.6 \\
\hline Inflation, end-of-period & 18.1 & 16.0 & 8.6 & 9.0 & 8.7 \\
\hline Exchange rate (average, Ghc per U.S. dollar) & 13.1 & 34.1 & 0.8 & $\ldots$ & \\
\hline & \multicolumn{5}{|c|}{ (Percent of non-oil GDP, unless otherwise specified) } \\
\hline Overall fiscal balance (cash) & -8.5 & -5.8 & -7.4 & -5.1 & -5.2 \\
\hline Overall fiscal balance (commitment) & $\ldots$ & -5.4 & -9.8 & -3.7 & -3.0 \\
\hline Central Government debt (net) & 30.1 & 32.7 & 43.1 & 42.7 & 44.0 \\
\hline Current account balance (percent of GDP) & -10.8 & -4.0 & -8.2 & -8.3 & -6.0 \\
\hline BOG GIR Stock (US\$ mill) & 2,036 & 772 & 4,725 & 5,432 & 6,390 \\
\hline Percent of prospective imports (G \& S) & 2.2 & 0.7 & 3.0 & 3.1 & 3.5 \\
\hline
\end{tabular}
some 65 percent, and it is now classified by the World Bank as a lower middle-income country.

2. Favorable economic setting in 2011. After a mixed performance in 2010, fiscal consolidation in 2011 has created room for interest rate reductions in support of robust economic performance. The government is now on track to reduce the fiscal deficit (after arrears clearance) by about $2 \frac{1}{2}$ percentage points of (non-oil) GDP, and the Bank of Ghana has reduced its policy rate twice this year-last to $12 \frac{1}{2}$ percent in July. Boosted by the start of oil production, overall economic growth is projected to reach $13 \frac{1}{2}$ percent this year and more than 8 percent in 2012, with average inflation expected to remain broadly unchanged at a rate of $81 / 2-9$ percent - well within the Bank of Ghana's target range (6.5-10.5 percent). The current account deficit is projected to remain broadly unchanged at $81 / 4$ percent of GDP, as high export growth from oil, cocoa, and gold, was matched by a strong rebound in both oil and non-oil imports reflecting buoyant economic activity. Supported by record foreign direct investment (FDI) inflows, official reserves are now projected to rise to $\$ 5.4$ billion at end2011 , covering just over three months of next year's imports.

3. Risks to outlook broadly balanced. External demand for Ghana's exports is expected to remain strong, with considerable upside potential over the medium term from newly discovered oil fields. Positive spillovers on domestic demand are likely to continue to feed into broad-based economic activity, creating possible upward pressure on inflation. The main downside risks on the external side arise from a possible weakening in world commodity prices and foreign investment flows as a result of a prolonged global slowdown - with increased risk aversion already evident in the recent depreciation of the cedi. On the domestic front, the main risk stems from possible public spending pressures ahead of the 2012 elections. 
Figure 1. Ghana: Real Sector Indicators, 2007-13
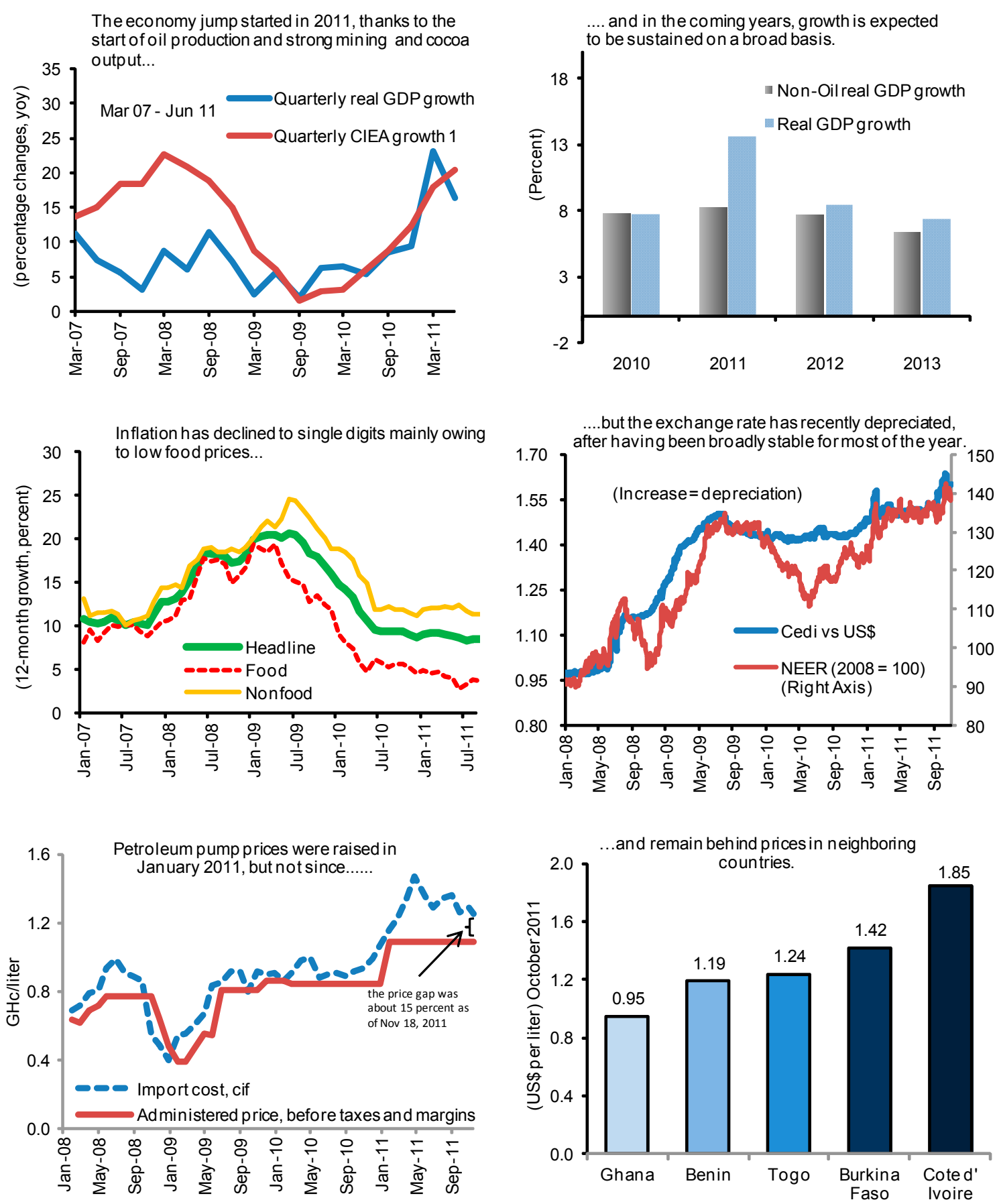

Source: Ghanaian authorities and IMF staff estimates and projections.

${ }^{1}$ The CIEA is the Bank of Ghana's composite index of real economic activity. 
Figure 2. Ghana: External Indicators, 2007-11
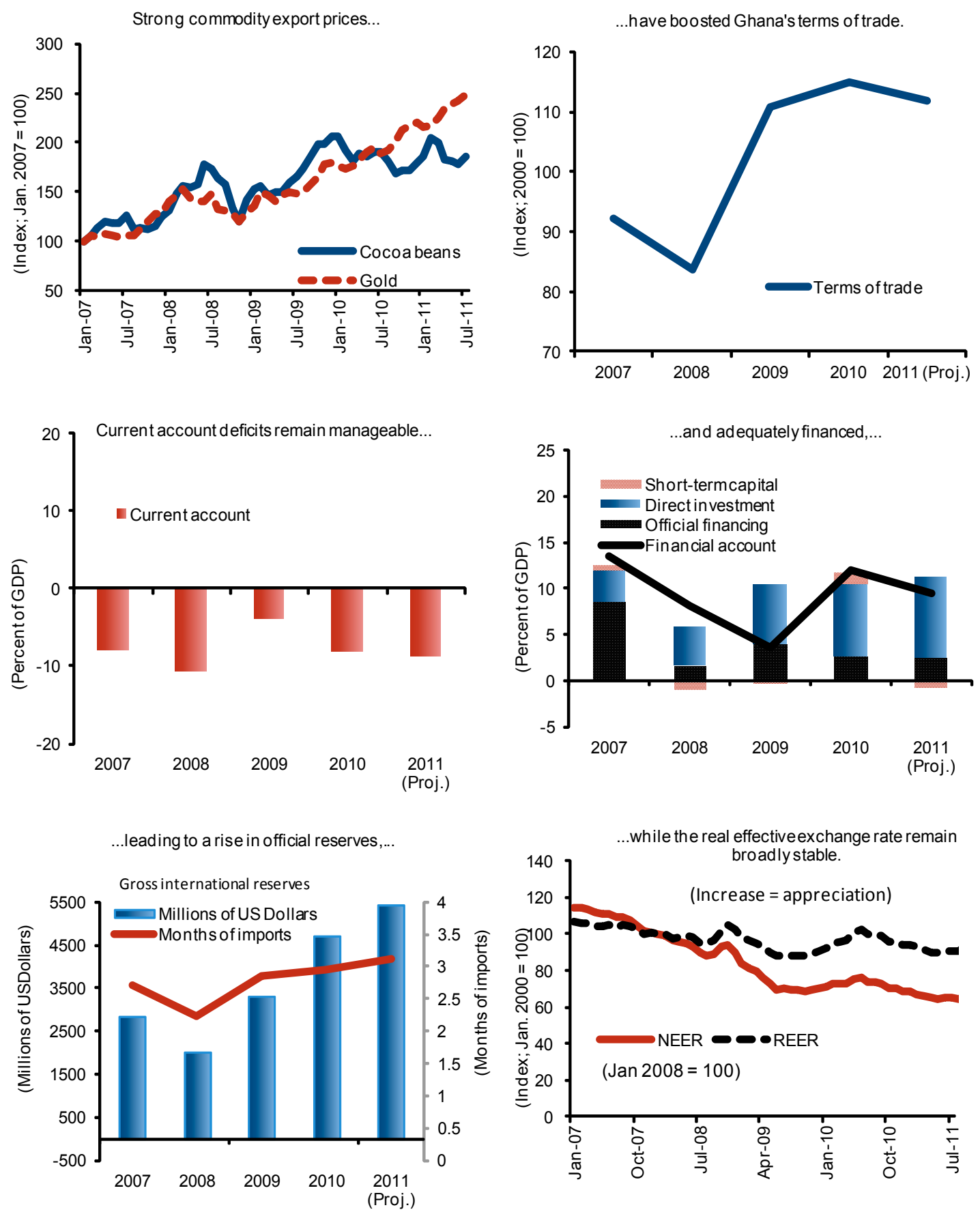

Source: Ghanaian authorities and IMF staff estimates. 


\section{B. Program Performance}

\section{Program performance has improved substantially in 2011:}

- All quantitative performance criteria and indicative targets for end-June 2011 were met, and preliminary data suggest a similarly favorable outcome for the end-September indicative targets; only the indicative target for arrears clearance was missed by a very small margin (MEFP Appendix Table 1).

- $\quad$ Structural reforms have been implemented with some unevenness (MEFP Appendix Table 2). Six benchmarks were met - two carried over from previous reviews - but four were missed. The most notable relates to petroleum price subsidies, which have begun to accumulate since August. Delays occurred in the rollout of Ghana's integrated financial management information system (GIFMIS) to 14 selected pilot ministries, reflecting a number of operational challenges. The reintroduction of quarterly expenditure ceilings was not met, because the authorities prefer to rely on monthly ceilings instead, until spending discipline is more firmly established. Finally, the independent audit of weak banks has been delayed, because the selection of the external consultant to oversee the process took longer than expected.

\section{The fiscal deficit target was met in both June and September, despite lower oil revenue and grants and higher wage payments (Figure 3 ).}

- Revenue. Non-oil tax revenue exceeded the program target through September, reflecting improvements in tax administration, including the segmentation of the taxpayer base to better target large taxpayers and the continued modernization of customs administration to improve the valuation of goods at the border. Figures based on collections show an even stronger improvement, with non-oil tax revenue exceeding the program target by GHф854 million. In addition, non-tax revenue benefited from gold shares sold in early 2011.

- Expenditure. A larger wage bill, reflecting a higher-than-budgeted base pay increase and the unexpectedly-high cost of migration to the new single spine salary structure (Box 1), was financed by restraint in other recurrent spending and lower capital expenditure.

\begin{tabular}{lrr} 
Text Table 2. Fiscal Performance, January-September 2011 \\
\cline { 2 - 3 } & \multicolumn{3}{c}{ Jan-Sept } \\
\cline { 2 - 3 } & \multicolumn{3}{c}{ (Millions of GHc) } \\
& \multicolumn{3}{c}{ Prov. } \\
Total revenue \& grants & 784 & 7,719 \\
Oil revenue & 5,429 & 6,067 \\
Non-oil tax revenue & 480 & 525 \\
Non-oil non-tax revenue & 1,065 & 621 \\
Grants & 9,065 & 8,215 \\
Total expenditure & 6,029 & 6,047 \\
Recurrent expenditure & 2,964 & 3,138 \\
$\quad$ Of which: Wages \& salaries & 691 & 648 \\
$\quad$ Goods \& services & 915 & 856 \\
$\quad$ Transfers & 312 & 258 \\
$\quad$ Reserve funds & 1,147 & 1,147 \\
Interest payments & 3,036 & 2,168 \\
Capital expenditure & $-1,307$ & -496 \\
Overall balance (before arrears) & -810 & $-1,068$ \\
Arrears clearance & -38 & -54 \\
Tax refunds & $-2,156$ & $-1,618$ \\
Budget balance (cash basis) & 318 & -486 \\
Discrepancy & $-2,473$ & $-1,132$ \\
Budget balance (incl. discrepancy) & 2,473 & 1,132 \\
Financing (incl. divestiture) & -572 & -572 \\
Divestiture & 1,432 & 460 \\
Foreign (net) & 71 & 29 \\
Exceptional financing & 1,542 & 1,215 \\
Domestic (net) & & \\
\hline Source: Ghanaian authorities and IMF Staff & &
\end{tabular}


Figure 3. Ghana: Fiscal Indicators, 2006-13
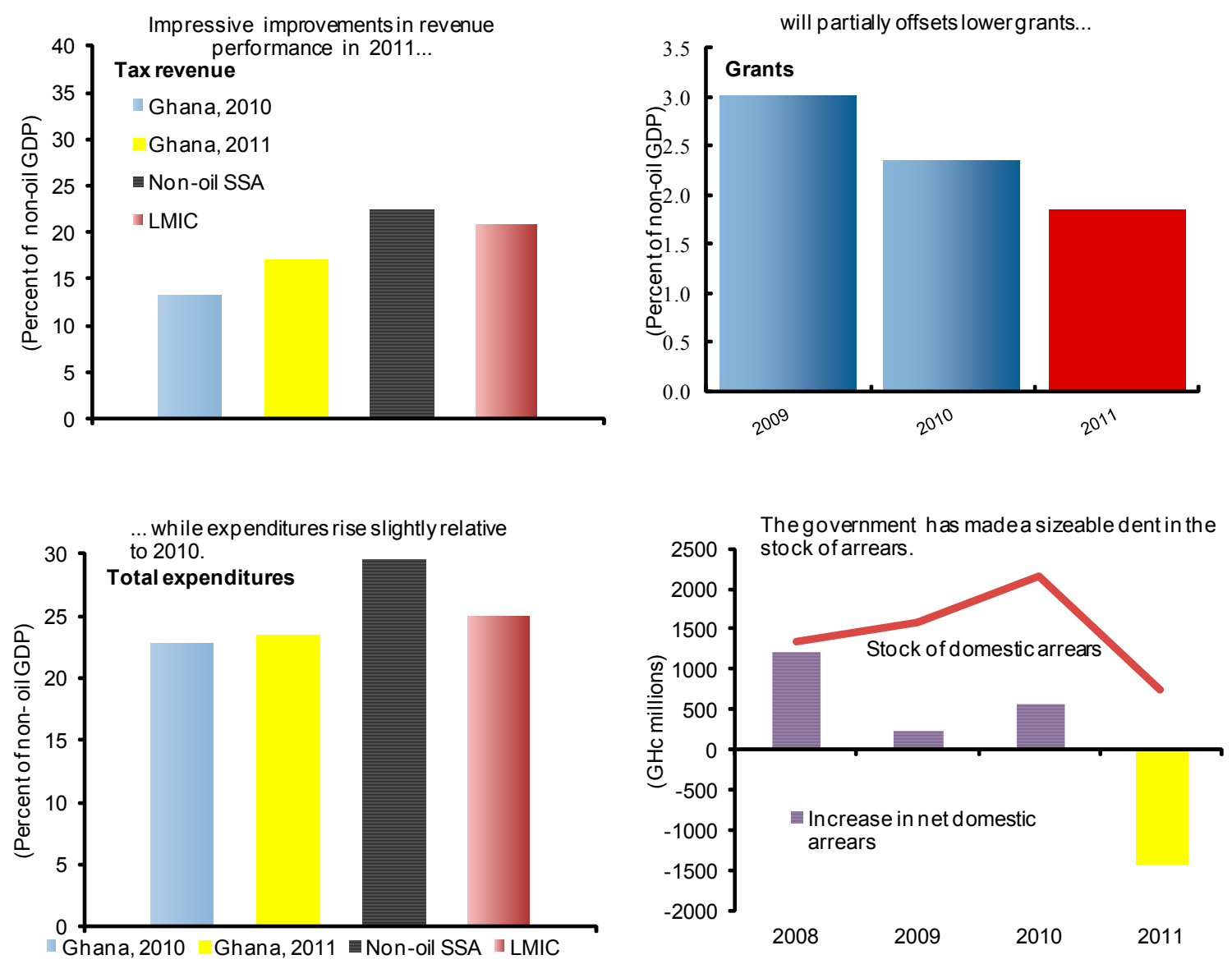

While thedeficit in 2012 is projected to decline further, it will exceed earlier targets...
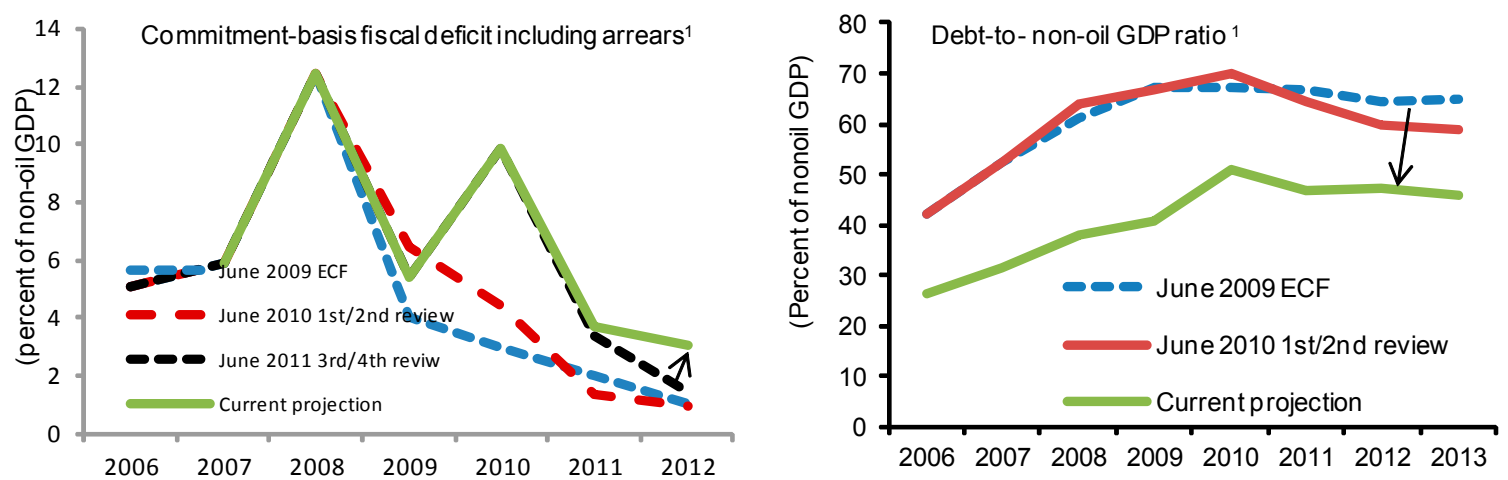

Source: Ghanaian authorities; and IMF staff estimates.

${ }^{1}$ Ghana's GDP was rebased in 2010. Current projection line uses the rebased GDP, while previous reviews are based on the old GDP series. 
- $\quad$ Balance. The overall deficit target (financing) was met by a large margin, partly because of a sizeable discrepancy (reflecting float transactions), but would have also been met otherwise.

\section{Box 1. The Single Spine Salary Structure and the Wage Bill}

- $\quad$ Management of the wage bill remains a major challenge. Cash wage payments are expected to be higher than programmed by 1 percent of non-oil GDP in 2011 and by 2 percent in 2012, reflecting two factors (Table 2A). First, the government awarded a 20 percent increase in base pay in July rather than the budgeted 13.5 percent (an additional 0.5 percent of non-oil GDP). Most of the unbudgeted increase, however, is the result of the migration to the single spine salary structure.

- $\quad$ In the medium term, the single spine structure should bring considerable benefits. These include more pay equity, easier salary negotiations, better oversight of outlays, and the potential for productivity gains. However, in the short term, it has raised the wage bill significantly.

- $\quad$ Estimating the cost of migration to the single spine has been difficult. The placement of employees into the new structure required time-intensive evaluations of job requirements, as well as negotiations. This has resulted in delays and the accumulation of sizeable deferred wage payments, retroactive to January 2010. Though 97 percent of public employees have now migrated, the final cost is still uncertain. The government currently estimates the stock of deferred payments to be GHф 1.25 billion (2.3 percent of non-oil GDP).

- $\quad$ The government plans to clear these deferred payments over a period of 10 months, with a first payment made in October 2011. Approximately GH\& 260 million (0.5 percent of non-oil GDP) will be paid in the fourth quarter of 2011, with the remainder paid by July 2012 .

\begin{tabular}{|c|c|c|c|c|}
\hline \multicolumn{5}{|c|}{ Central Government Wage Bill } \\
\hline & 2010 & 2011 & 2012 & 2013 \\
\hline & Act. & Proj. & Proj. & Proj. \\
\hline & \multicolumn{4}{|c|}{ (percent of non-oil GDP) } \\
\hline Wage commitment (after single spine, post-audit) ${ }^{1}$ & 8.1 & 9.3 & 7.8 & 8.1 \\
\hline Cash payment & 6.9 & 8.5 & 9.3 & 8.1 \\
\hline Deferred wage payment (net) & 1.2 & 0.8 & -1.5 & 0.0 \\
\hline Accumulation of deferred payments & 1.2 & 1.3 & 0.0 & 0.0 \\
\hline Clearance of deferred payments & 0.0 & 0.5 & 1.5 & 0.0 \\
\hline Stock of deferred wage payments & 1.2 & 1.8 & 0.0 & 0.0 \\
\hline
\end{tabular}


6. Based on the performance through September, the 2011 fiscal deficit is likely to be smaller than programmed, by about $1 / 2$ percent of GDP. This corresponds to a significant adjustment from 2010 of 6 percentage points of non-oil GDP on a commitment basis (Text Table 3). Strong revenue performance in 2011 is largely responsible for the substantial improvement in the commitment-based deficit, but spending has also declined, as a share of non-oil GDP, except for wages. The 2011 projections incorporate seasonally strong tax revenue in the fourth quarter, with corporate taxes likely to receive a further boost from the exhaustion of capital allowances in the mining sector. However, the shift in the composition of spending, with lower foreign-financed capital spending and higher wage payments, will result in larger domestic financing (by $1 \frac{1}{2}$ percent of GDP) than initially programmed.

\begin{tabular}{lrrr}
\multicolumn{3}{c}{ Text Table 3. Central Government Operations (commitment basis) } \\
\hline & 2010 & 2011 & 2012 \\
\cline { 2 - 4 } & Act. & Proj. & Proj. \\
\cline { 2 - 4 } & & & \\
& 16.6 & 21.0 & 21.5 \\
Total revenues and grants (net of VAT refunds) & 14.4 & 19.3 & 19.9 \\
Revenues & 2.3 & 1.8 & 1.8 \\
Grants & -0.1 & -0.1 & -0.1 \\
VAT refunds & & & \\
& 26.5 & 24.7 & 24.6 \\
Total expenditure (commitment basis) & 16.3 & 17.4 & 15.8 \\
Recurrent expenditure & 8.1 & 9.3 & 7.8 \\
$\quad$ Wages and salaries & 8.2 & 8.1 & 8.1 \\
$\quad$ Other recurrent expenditure & 7.5 & 6.8 & 8.8 \\
Capital expenditure & 2.6 & 0.0 & 0.0 \\
New arrears & 0.0 & 0.5 & 0.0 \\
Commitments related to float transactions 1/ & & & \\
Budget balance (commitment basis) & -9.8 & -3.7 & -3.0 \\
\hline Sources: Ghanaian authorities and IMF staff projections. & & & \\
1/ Contingency included in 2012 budget to meet float transactions related to 2011 commitments.
\end{tabular}

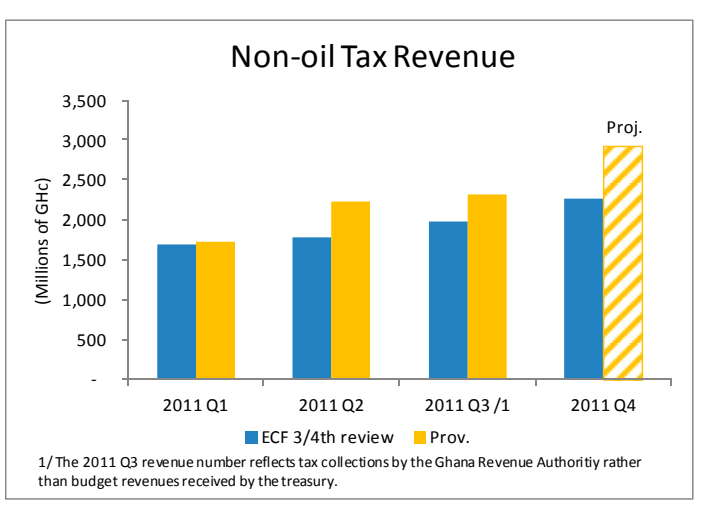

\section{Policy Discussions}

Discussions focused on policies to preserve macroeconomic and financial stability, while implementing high-priority investments to spur long-run growth and development.

\section{A. Setting the Stage for Scaled-Up Investment}

7. Agenda for growth and development. Addressing large infrastructure gaps, is widely seen as a prerequisite for assuring Ghana's long-term growth and is a focus of the Ghana Shared Growth and Development Agenda (GSGDA) for 2010-13. At the same time, Ghana's access to concessional financing will be tapering off, given its new middle-income status. Against this background, the government has recently negotiated a US\$3 billion (8 percent of GDP) "Master Facility Agreement" (MFA) on nonconcessional terms from the China Development Bank (CDB) to finance a number of critical infrastructure investments, with disbursements expected to be stretched over four years (Box 2, and MEFP 97). To 


\section{Box 2. Master Facility Agreement}

Background. The government of Ghana has negotiated a \$3 billion (8 percent of 2011 GDP) Master Facility Agreement (MFA) with the China Development Bank (CDB). The agreement, which has not yet been signed, covers various public investment projects, including railways, gas infrastructure, industrial zones in the western region, commercial agriculture, multi-modal transportation in the eastern region, fisheries infrastructure in coastal areas, urban transport in Accra, and nationwide SME incubation. All align with the priorities of the GSGDA. Project implementation will be supervised by the respective ministry, and debt service payments will be from the ABFA (Box 3). Associated capital spending is currently captured in the budget, but the government plans to on-lend these funds to state-owned enterprises, at which point capital expenditure items funded by the MFA will be reclassified as net lending. Subsidiary loan agreements for each project financed under the MFA will require approval by the CDB and Ghana's parliament before disbursements are made.

Terms and conditions. The loan has two tranches of equivalent amounts, one spanning 15 years (Tranche A) and the other 10 years (Tranche B), with a grace period of 5 years. Both tranches are provided on nonconcessional terms (6-month LIBOR +295 bps for Tranche A, 6-month LIBOR + $285 \mathrm{bps}$ for Tranche B) and include a 1 percent commitment fee on signing of the MFA and counterpart funding (15 percent). The MFA further stipulates that contractors from the People's Republic of China must implement a minimum of projects equal to 60 percent of the loan amount.

\section{World Bank assessment:}

$>$ At the government's request, the World Bank has assessed the financial facility under the MFA and related projects. Its report notes that project documents provide a substantial information base to complete the necessary feasibility studies, which in some cases will need to be updated, because the coverage of projects has changed. In other cases, existing feasibility studies conclude that projects are marginally viable from a financial perspective. In a few cases, data shared with the World Bank do not amount to feasibility studies, but constitute useful preliminary information to initiate them.

$>$ Among the list of projects, the gas infrastructure project is the one that deserves the highest and most urgent levels of attention. The justification for the gas project retained under the MFA, for about US\$1 billion, is sound and economic returns on it would be even larger, if the project included the infrastructure for LPG production. In this case, the much larger returns would alone cover 80 percent of the financial costs to be incurred on the entire US $\$ 3$ billion amount of the MFA. Beyond covering almost the entire loan, such high returns could be used to cross-subsidize some of the other projects, if expected social returns are deemed to justify this.

$>$ World Bank staff believes that the MFA provides a significant opportunity for Ghana to advance its Shared Growth and Development Agenda. It recommends that the government take the necessary time to finalize feasibility studies, with priority being given to the completion of the thorough preparation of the gas project. By doing so, risks of macroeconomic disruption would be minimized, and the opportunity would be given to strengthen the institutional framework for public investment decisions. 
accommodate this loan under the program, they requested a modification of the endDecember 2011 performance criterion on the contracting of nonconcessional external debt, to raise the limit from $\$ 800$ million to $\$ 3,400$ million. This would cover the MFA and all other nonconcessional loans contracted under the existing ceiling.

8. Scope for nonconcessional borrowing. While recognizing the importance of scaling up infrastructure investment, the mission stressed the need to preserve debt sustainability and recommended a careful assessment of the cost and benefits of the underlying projects and the financing arrangements. To inform the decision on modifications to the borrowing ceiling, a new debt sustainability analysis (DSA) was prepared, jointly with the World Bank, incorporating the authorities' plans for increased nonconcessional borrowing to scale up infrastructure investment. In conclusion, staff supports the requested modification based on:

i. The DSA. The analysis confirms that risks remain manageable, even under conservative assumptions on growth dividends, but contingent on successful fiscal consolidation, as envisaged under the program.

ii. The World Bank assessment. World Bank staff view the MFA as a significant opportunity for Ghana to advance its development agenda. At the same time, they recommend a gradual implementation of the projects, with the government taking the necessary time to finalize feasibility studies and giving priority to the gas project which promises significant returns.

iii. The urgency of the gas-pipeline project. Gas is produced as a side product in the oil production, but is currently not usable. Apart from the commercial benefits of gas utilization, the pipeline is needed urgently to avoid the significant risks to existing oil wells of gas-reinjection or environmentally damaging flaring.

\section{There was agreement that reliance on significant nonconcessional financing} called for a stronger institutional framework for public investment decisions. In this context, the authorities agreed to the establishment of certain principles that would guide their drawdown and future contracting of nonconcessional debt (MEFP $q 24)$ :

- $\quad$ Disbursement profile. The government will ensure that the drawdown of resources does not exceed the planned disbursement schedule incorporated in the program and the DSA. In particular, the drawdown of nonconcessional public or publicly guaranteed debt will be about $\$ 1,250$ million in 2012, of which $\$ 750$ million will be direct central government borrowing related to the MFA. The remaining MFA funds are expected to be drawn in the same annual amounts of \$750 million over 2013-15.

- $\quad$ Project assessments. The government will ensure that each project is supported by robust feasibility and financial viability studies, confirmed by reliable third-party assessments. 
- Integrated investment planning and debt management. The authorities will establish policy guidelines, by April 2012 (new structural benchmark, Appendix Table 2 of the MEFP), to improve coordination between the Public Investment Division, the Debt Management Unit, and the Economic Research and Forecasting Division of the Ministry of Finance and Economic Planning (MoFEP). The objective is to strengthen the institutional framework for making public investment decisions - from strategic guidance and formal appraisal, to project selection, budgeting, implementation, and evaluation - and to ensure that the contracting of debt is guided by the medium-term macroeconomic framework.

10. While adherence to these principles should help contain important risks, the mission also stressed the need for caution and fiscal prudence. It will take some time for a robust institutional framework to be effective, and project risks - including delayed implementation, cost overruns, and failure to generate expected returns - should be factored into the planning. On the implementation side, the mission was encouraged by decisions to hire internationally recognized engineering and construction firms to conduct front-end engineering of the gas project, and by plans to employ project management consulting firms to support the public entity in charge of the project. On the macroeconomic and debt sustainability side, however, risks will also depend on the phasing of projects and the ability to contain fiscal deficits. ${ }^{1}$

\section{B. Fiscal Policy: Preserving Gains to Macroeconomic Stability}

Discussions focused on the need to create fiscal space for higher investment spending and maintain spending discipline in the run-up to elections.

\section{The 2012 program seeks to balance the need for high-priority investments to} close the infrastructure gap with the importance of maintaining fiscal discipline. The program targets a total deficit of 51/4 percent of non-oil GDP for 2012 , up by $23 / 4$ percentage points relative to the last review (Table 2). This would still reduce the commitment-based deficit by about $3 / 4$ percentage point of non-oil GDP, despite a 2 percent of GDP increase in foreign-financed capital expenditure and poverty-related spending of about $5 \frac{1}{2}$ percent of GDP, consistent with GSGDA objectives. The government's fiscal strategy thus hinges on a further increase in revenue - which are low in relation to the size of the economy - and determined efforts to control nonpriority spending and strengthen public financial management. However, to guard against the risk of fiscal slippages, the authorities noted that they would hold back on appropriations on goods and services and domestically-financed

\footnotetext{
${ }^{1}$ A scaling-up exercise prepared for Ghana in 2009 suggests that the implications of higher spending on macroeconomic stability and inflation likely will be manageable (the exercise assumed a $4 \frac{1}{2}$ percent increase a year in foreign inflows). However, inflation risks rise when a substantial portion of the spending is directed toward non-traded goods.
} 
capital spending until uncertainties about the outcome of the payroll audit and implementation of new tax measures are resolved.

\section{Raising revenues}

12. Tax administration has improved substantially, and the authorities plan further reforms. As shown by strong tax performance in the first three quarters of 2011, the government's past administrative reforms - including the establishment of a large taxpayer office, streamlining of tax exemptions, and strengthened customs administration - are paying off. Work is underway to further improve tax administration (MEFP $\$ 10-16$ ), and a new VAT bill—submitted to parliament in May 2011 (a prior action under the previous review), but not yet passed - is expected to yield some 0.2 percent of non-oil GDP in additional revenue. The mission urged the authorities to seek accelerated approval of the bill, which includes the extension of VAT to fee-based financial services.

\section{The mission strongly welcomed the government's plan for new tax policy} measures, with an expected yield of at least 0.8 percent of GDP (MEFP |18-22). In line with established practice in the oil and gas industry and in peer resource-rich countries, the corporate tax rate for mining will increase from 25 to 35 percent. For those two mining companies that have stability agreements, the tax increase requires consent, which the government is confident to receive. Furthermore, the government will implement a windfall profit tax in the mining sector of 10 percent on profits above a certain threshold that remains to be determined. The 2012 budget will also establish a uniform tax regime for capital allowances, inaugurate new rules to ensure ring fencing of mining projects, and adopt OECD guidelines for transfer pricing. In addition, the government plans to review the existing legal framework for capital gains taxes, to enhance its ability to collect revenue following the transfer of interest between nonresidents. Increased collections are also expected from the payment of personal income tax on deferred wages to civil servants. Some measures will reduce the tax burden; the national fiscal stability levy will be eliminated, honoring an earlier commitment, and the tax holiday for the Ghana Stock Exchange will be extended by another five years. The projected yield of 0.8 percent of GDP from new tax measures is a conservative estimate. It does not include any revenue gains from the windfall profit tax, uniform tax regime on capital allowances, new rules on transfer pricing, or changes in capital gains taxes, which remain to be quantified.

\section{Managing the public wage bill}

\section{There was agreement that careful management of the wage bill will be essential} to continued success of the program (MEFP |25-27). The authorities plan to clear all remaining deferred wage payments in the first seven months of 2012. At the same time, they are determined to keep the wage bill, on a commitment basis, broadly constant in nominal terms. To this end, the authorities expect to offset a first increase in pay relativity since the inception of the single spine with savings from a payroll audit-projected at 8 percent of the 
2011 wage bill - to be completed by mid-2012 (structural benchmark). Any base pay raises would then be contingent on savings over and above the 8 percent from the audit. The mission urged a more gradual phasing of deferred payments over a period of two years, in light of limited budgetary flexibility and constraints on domestic financing. The authorities agreed that a phased approach would be preferable on economic grounds, but noted the importance of delivering on their commitment to civil servants during the government's current term. They also believed that the savings from the payroll audit will likely exceed the assumed 8 percent of the wage bill. Early results from a pension audit showed savings of 25 30 percent.

15. The authorities recognized risks to the fiscal outturn, but felt that the current projections incorporated sufficient buffers. They pointed, in particular, to their conservative revenue estimates, which did not include gains from a number of measures. Nevertheless, in the event of budget overruns in excess of additional revenue, the authorities expressed their commitment to protecting the fiscal targets by tightening appropriations to spending units for both goods and services and delaying domestically financed capital spending. The mission agreed that there was limited room to adjust spending other than through ad hoc cuts. A major difficulty is the earmarked allocation of revenue to specific funds. Although changes to these rules require constitutional amendments, the mission encouraged the authorities to seek ways to address this issue over the medium term.

\section{Strengthening public financial management}

\section{The government has made substantial progress in reducing its domestic} payment arrears, which should be cleared fully by 2013 (MEFP $\mid 28$ ). The government's arrears strategy involves the combination of cash payments and the issuance of special purpose bonds and promissory notes. The Budget Department of the MoFEP tracks flow payments in a comprehensive database, with monthly status reports presented to the cabinet. To prevent the emergence of new arrears, a presidential circular has mandated all line ministries to secure commencement certificates before committing the government to any contractual obligation. As a result, the authorities have reported no new arrears in 2011.

\section{The government plans to continue monitoring spending through monthly} expenditure ceilings (MEFP \$29-30). Although there was agreement that quarterly ceilings would provide additional flexibility and planning certainty to ministries and departments, the authorities preferred postponing the ceilings' introduction until spending discipline has been firmly established. The mission accepted the current priority in favor of tighter controls. It also welcomed the communication of indicative spending ceilings ahead of the 2012 budget preparation process, based on the new unified chart of accounts to support the establishment of realistic spending plans.

\section{The mission stressed the importance of prudent cash management, noting} challenges evidenced by the large float transactions in the first half of 2011 
(MEFP 931). Although a treasury main account exists, the government's cash balances are held in numerous accounts at the Bank of Ghana and commercial banks. This has led to situations, as in June, where the treasury main account had insufficient funds to affect payment, creating a discrepancy between the deficit and total financing. The mission suggested stronger efforts to eliminate unnecessary bank accounts and accelerate the migration to a treasury single account (TSA). The authorities agreed to continue the transition to a TSA, but saw a need for many of the existing accounts, including those for donor projects. They expressed confidence that a new operational framework, developed with the Bank of Ghana to match upcoming payments more closely with account balances, would help reduce the occurrence of large discrepancies. They also pointed out that the sizeable June discrepancy was almost halved by September.

\section{Energy Policies: Responsible Management of Revenues and Prices}

19. The adoption of the Petroleum Revenue Management Act (PRMA) in April 2011 has been a pivotal first step toward transparent and responsible revenue management. The PRMA establishes a strong legal framework for the collection, allocation, and management of petroleum revenue in a transparent and accountable manner (Box 3). The bill benefited from broad consultations with many stakeholders, including civil service organizations, before enactment. The authorities are now drafting supporting regulations, detailing rules, responsibilities, and administrative procedures.

20. Quarterly reviews of power tariffs have continued, but did not always result in adjustments to cost-recovery levels (MEFP \|33). The Public Utilities Regulatory Commission (PURC) has used its adjustment formula to establish tariff levels. Accordingly, it reduced electricity tariffs in March 2011 and increased them in September 2011. The PURC did not adjust tariffs in June 2011, despite calculations that an increase of about $81 / 2$ percent was warranted, because utilities' service delivery had deteriorated and expected efficiency gains were not achieved. While acknowledging the need to maintain incentives for utilities to improve their operational efficiency, the mission welcomed the subsequent price adjustment to cost-recovery levels in September.

\section{Gains from petroleum price hedging operations have provided temporary} protection from global price increases, but are now exhausted. Pump prices have not been adjusted since a 30 percent rise to cost-recovery levels in January 2011. The authorities avoided fiscal costs for half of the year by covering the subsidies through hedging gains, as intended. However, failure to adjust prices subsequently (September 2011 benchmark) led to costs of about GHф 100 million ( 0.2 percent of non-oil GDP) by October. Encouraged by the mission to take quick corrective actions, the authorities agreed to repay the outstanding amounts from the budget and to adjust petroleum prices on a monthly basis, 


\section{Box 3. Petroleum Revenue Management Act}

The Petroleum Revenue Management Act (PRMA) incorporates several provisions to ensure a strong legal basis for the effective and efficient application of petroleum revenue in Ghana, including:

- Establishment of petroleum funds and allocations: All petroleum revenue is received and disbursed via a dedicated Petroleum Holding Fund (PHF), held at the Bank of Ghana. Revenue from the PHF is disbursed into three separate funds: the Consolidated Fund to support the annual budget; the Stabilization Fund to cushion the budgetary impact of annual volatility in oil revenues,; and the Heritage Fund to provide an endowment for future generations. The latter two receive from the PHF all revenue in excess of the Annual Budget Funding Amount (ABFA).

- Benchmark revenue: Benchmark revenue is set by September of each year for the budget of the following year. It is determined on the basis of a five-year rolling average of oil prices.

- Annual Budget Funding Amount (ABFA): This is the amount of petroleum revenue provided to the annual budget. The ABFA cannot be more than 70 percent of benchmark revenue. The exact percentage is determined annually and approved by parliament. The ABFA is considered part of the national budget, and its use is subject to the same budget processes, e.g., guided by mediumterm expenditure plans and aligned with the national development plan (the GSGDA). Moreover, a minimum of 70 percent of the ABFA annually must be used for public investment expenditures.

- Collateralization: The PRMA permits the use of the ABFA as collateral for debts and other liabilities of the government for a period of up to 10 years after its commencement. The PRMA prohibits borrowing against the amounts earmarked for the Stabilization and Heritage Funds.

- Reporting: The Act provides for reporting on various levels. The reporting authorities include: (i) the Ghana Revenue Authority; (ii) the Ministry of Finance and Economic Planning; (iii) the Bank of Ghana; (iv) the Investment Advisory Committee; (v) the Auditor-General; and (vi) the Public Interest and Accountability Committee. Based on their mandates, reports are due either monthly, quarterly, semi-annually, or annually.

- Transparency and accountability: Transparency clauses are consistent with the requirements of the Extractive Industry Transparency Initiative. A strong framework for public accountability is ensured through disclosures of public expenditures and the regular scrutiny by the Public Interest and Accountability Committee. The Accountability Committee must publish semi-annual and annual reports in two state-owned newspapers, post the report on its website, and hold meetings twice a year to discuss the reports with the public. The Committee also has to submit a copy of its semi-annual and annual reports to the president and to parliament. The auditor-general provides external audits of the petroleum funds each year, while the Bank of Ghana conducts internal audits, with the governor submitting quarterly reports. 
to return to full cost-recovery levels (MEFP $\left\{33\right.$ ). ${ }^{2}$ The authorities have further agreed to implement more frequent price adjustments going forward, to prevent fiscal costs and depoliticize the pricing process. Additionally, to ensure transparency, the profits (or losses) from the hedging program will be included in the 2012 budget.

\section{Monetary and Financial Policy: Safeguarding Low Inflation and Financial Stability}

Discussions focused on the need to guard against inflation risks and the implementation of measures, recommended in the FSAP update, to strengthen the financial sector.

\section{Monetary policy implementation has been consistent with objectives of the 2011}

program (Figure 4). Despite a larger-than-expected rebound in broad money, mainly owing to higher net foreign assets and growth in credit to the private sector, inflation remained stable, helped by low food inflation. During the fourth quarter, greater reliance will have to be placed on open market operations and foreign exchange interventions to sterilize the seasonal liquidity generated by cocoa board transactions. The mission supported a policy of targeted intervention to smooth excessive exchange rate volatility and manage liquidity, while allowing the exchange rate to adjust to more lasting trends. In light of the high cost of sterilization operations, the mission urged swift adoption of a plan to share the cost of sterilization with the budget, while stressing more generally the importance of effective coordination of fiscal and monetary policy in maintaining low inflation.

\section{The authorities are confident about achieving their 9 percent inflation target by} the end of 2011 and keeping inflation in single digits in 2012. As the economy and financial services are expanding at a rapid pace, they expect the trend decline in velocity to continue, even without further disinflation (see text chart). Hence, they expect that broad

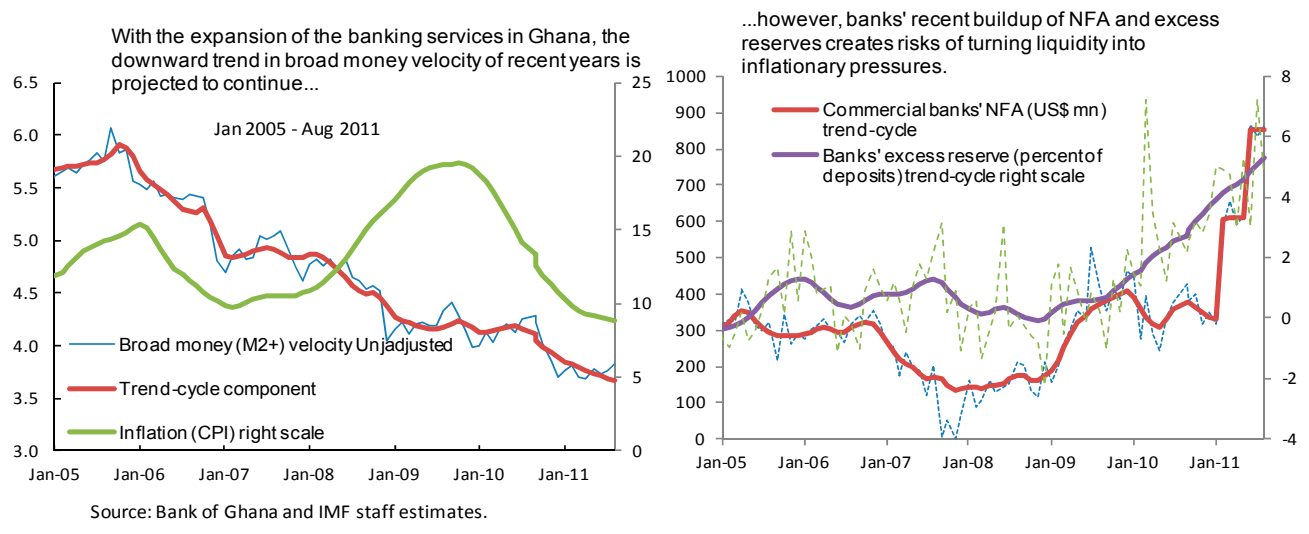

\footnotetext{
${ }^{2}$ As of November 18, data for world petroleum prices suggest that a 15 percent increase would be sufficient to bring pump prices back to cost-recovery levels.
} 
Figure 4. Ghana: Financial Indicators, January 2008 - October 2011
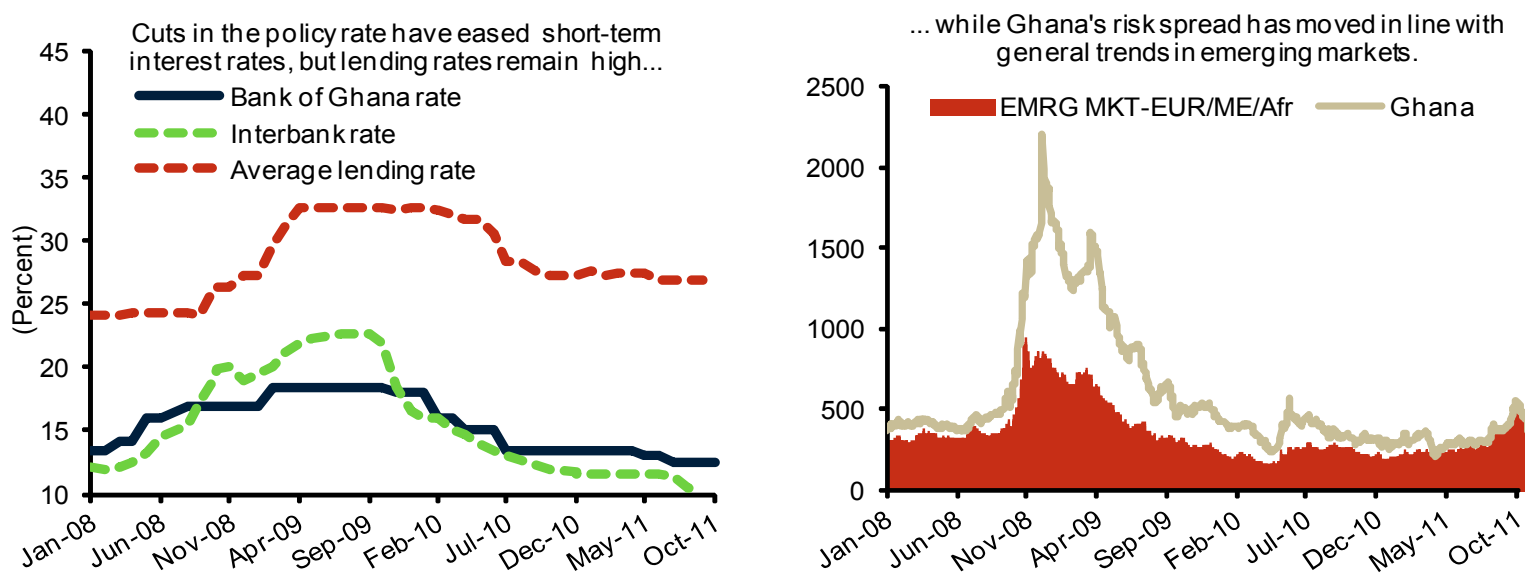

On average, banks are well capitalized..
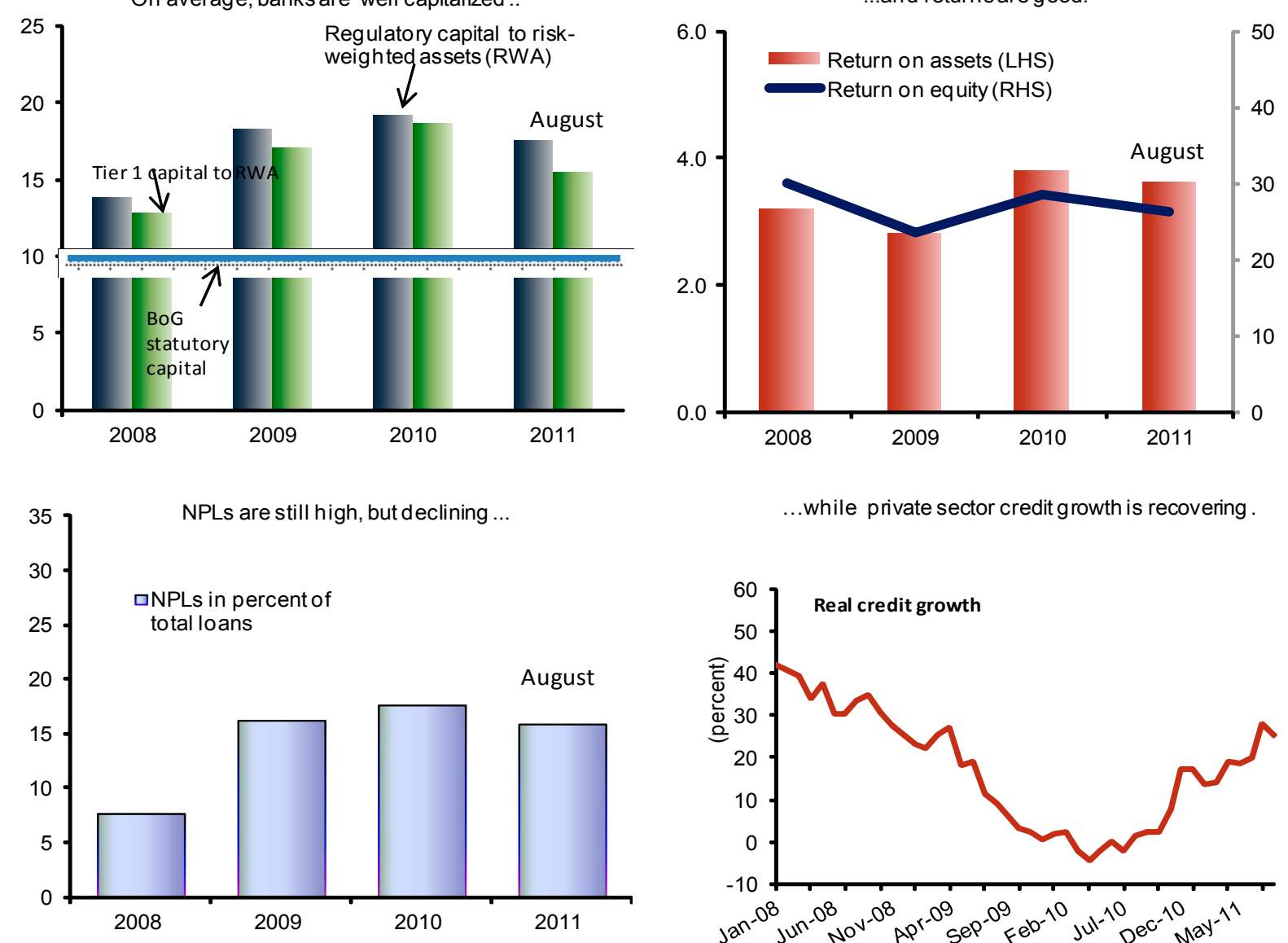

...while private sector credit growth is recovering .

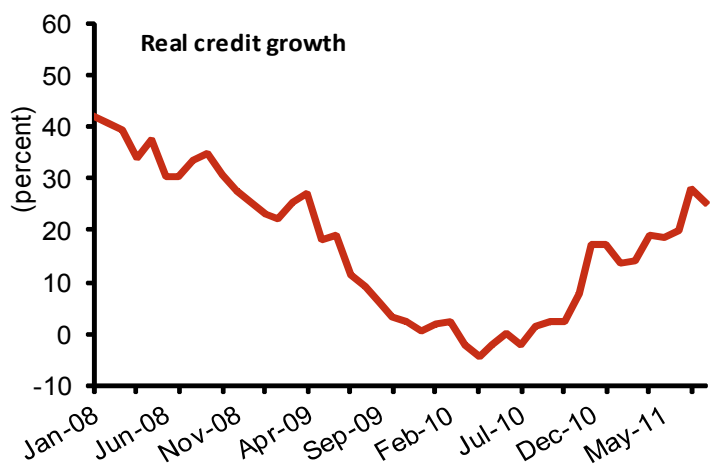

Source: Ghanaian authorities; DataStream; and IMF staff estimates. 
money growth of 35-45 percent will still be consistent with the targeted inflation trajectory, while providing ample room for increased credit to the private sector. The authorities concurred with the mission, however, that tightening monetary policy may be needed in the months ahead, and agreed to adjust policy rates should upside risks to inflation become acute.

\section{In the banking sector, NPLs are declining, but are still high at 16 percent.}

Commercial bank lending rates should continue to decline as inflation expectations are lowered, while nonperforming loans are being brought under control and current risk concerns ease. On aggregate, the banking sector remains adequately capitalized, and all banks have met the minimum capital requirement of GH\&25.0 million and are expected to comply with the $\mathrm{GH} \notin 60$ million requirement by end-2012. Banks' liquidity positions remain satisfactory, while asset quality has improved.

25. Reform priorities include strengthening banks' risk management capacity, resolving remaining problem banks, and enhancing the BoG's supervisory capacity. Several specific actions are underway (MEFP $\mid 36$ ), including (i) improved risk management practices across the banking industry, and in particular at the Ghana Commercial Bank; (ii) selection of a long-term advisor expected to take office in January 2012 to help BoG build capacity in banking supervision and oversee the auditing process for the problem banks, with the planned appointment of firms to conduct the audit by end-March 2012 (structural benchmark); (iii) contracting of legal consultants to amend the Banking Act and other related regulatory acts; and (iv) successive removal of the BoG from ownership in banks to resolve the potential conflict of interest.

26. The mission urged further action to address remaining deficiencies in its AML/CFT regime. An AML office was established within the Banking Supervision Department in February 2011, and resources are being recruited to make it fully operational. The office has been working on guidelines for the banking industry and is receiving technical assistance from the Fund to strengthen the overall legislative and regulatory AML/CFT regime. However, the Financial Action Task Force is not yet satisfied with Ghana's progress in addressing its AML/CFT deficiencies. To avoid any repercussions of a negative assessment, staff urged the authorities to continue improving its AML/CFT regime, drawing also on Fund technical assistance.

\section{Program Monitoring, Modification of Performance Criteria, And Risks}

\section{The authorities request, with staff support, modification of the quantitative} targets for end-December 2011 through end-June 2012. The proposed performance criteria and indicative benchmarks are presented in Appendix Table 1 of the MEFP. In particular, the authorities request a lowering of the floor on GIR for end-December 2011 by US\$271 million, to reflect lower concessional official transfers and oil-related inflows. They also request an increase of the 2011 ceiling on the contracting of medium- and long-term external debt on nonconcessional terms from US\$ 800 million to US\$3,400 million, to 
provide higher financing for scaled-up infrastructure investment. Staff's support of this modification reflects the broadly favorable conclusions of an updated debt sustainability analysis and the agreed measures to ensure proper project assessments and appropriately phased disbursements.

28. Program monitoring for the rest of the program will be on a quarterly basis.

Appendix Table 2 of the MEFP shows the proposed prior action and structural benchmarks. Three new benchmarks are proposed to be added: on completing a civil service payroll audit; improving coordination in the area of debt management; and conducting independent audits of remaining problem banks. It is further proposed that the introduction of quarterly expenditure ceilings be dropped as a structural benchmark; while the authorities still see merit in this measure, they prefer to rely on monthly ceilings instead, until spending discipline is more firmly established. Finally, it is proposed to reset the benchmark on managing petroleum product prices on a recurrent basis to avoid fiscal subsidies.

\section{Risks to the program remain manageable, provided policy commitments are} sustained. Program risks arise, on the external side, from the possibility of a weakening in world commodity prices and foreign investment flows, in response to a prolonged global slowdown. On the domestic side, the main risks arise from an overrun in the wage bill, in the event insufficient savings from the payroll audit, as well as to whether the recent progress in revenue collection can be sustained. These remain manageable, in terms of the underlying program objectives, provided the authorities resist pressures to relax the fiscal stance ahead of the 2012 election.

\section{Staff Appraisal}

30. Program performance has improved significantly in 2011. Taking advantage of a favorable economic environment, the government has made great strides in boosting domestic tax revenues and repaying arrears. The management of the wage bill, however, has proved challenging, in light of the unexpectedly high cost of moving public sector employees onto a new salary structure, but also larger-than-programmed base pay increases. Thus, while the fiscal deficit target is within reach, current spending will exceed earlier targets, creating risks that inflationary pressures could reemerge.

\section{Investments in infrastructure are crucial for Ghana's sustained growth, but} projects and their financing should be planned and monitored carefully. A revised debt sustainability analysis suggests scope for higher nonconcessional borrowing, and some of the planned projects promise significant returns. Staff, therefore, supports a modification of Ghana's nonconcessional borrowing limit to accommodate the authorities' borrowing plans. At the same time, it is crucial that all projects are supported by robust feasibility studies and that their implementation and associated disbursements be phased in consistent with program targets to preserve fiscal and debt sustainability. Moreover, Ghana's increasing reliance on nonconcessional financing will require a stronger process of integrated investment planning 
and debt management that should be rigorously applied to all future projects and investment decisions.

\section{The government's plans for raising tax revenue are welcome and important for} creating fiscal space. Recent success in mobilizing revenue should offer strong encouragement to continue important reforms in tax administration. In addition, the government is right to look for new tax policy measures to raise Ghana's revenue ratio from a still low level, relative to the size of its economy. In this context, the planned menu of measures is a welcome step, particularly in natural resources, where taxation is low in comparison with peer countries. Prompt parliamentary passage of new legislation to broaden the value added tax (VAT) base will also be important.

\section{On the expenditure side, management of the wage bill remains a challenge and} risk. Although the adoption of a uniform public sector pay structure promises important benefits over time, it has also been costly. And the plan to clear deferred wage payments by mid-2012 will likely prove challenging, even without any base pay increases, unless expected savings from the payroll audit can be realized quickly. Over the medium term, improvement of the structure, and reduction of the size, of public sector employment will be important to realizing the hoped-for efficiency gains. In the meantime, offsetting savings will have to come from restraint in other spending, supported by ongoing improvements in public financial management - where some reforms have taken longer than expected.

\section{While energy pricing has improved, decisive action is needed to tackle the} reemergence of subsidies on petroleum products. The PURC's September decision to raise electricity and water tariffs to cost-recovery levels was a welcome sign that the quarterly review process is working. This is in contrast to the failure to adjust petroleum prices, despite rising international prices and an exhaustion of hedging gains by midyear. While hedging operations offer valuable insurance against excessive price fluctuations, they cannot be relied upon to generate profits. Thus, more frequent price adjustments to cost-recovery levels are essential to avoid costly and poorly targeted subsidies and helpful to depoliticize the process. The authorities' renewed commitment to such an approach, and their plan to ensure transparency of the hedging program through the budget, are welcome.

\section{The Bank of Ghana has earned credibility in meeting its inflation target. With} upside risks to inflation dominating, it should now stand ready to raise rates promptly as signs of rising price pressures emerge. In light of considerable volatility in the foreign exchange market, it will also be important for the BoG to further refine, and better communicate, its policies and instruments for foreign exchange market interventions. Moreover, to manage liquidity effectively, the BoG will need the support of the budget to ensure the availability of sufficient resources for conducting sterilization operations. 
36. Financial sector reforms should continue with renewed zeal. The BoG should focus its attention on making further progress in areas identified in the FSAP update, including strengthening banks' risk management, resolving vulnerable institutions, and enhancing its supervisory capacity. Addressing promptly the identified deficiencies in its AML/CFT regime will also be important to avoid reputational risks.

37. In light of Ghana's strong performance in 2011 and policy intentions for 2012, staff recommends completion of the fifth review under the ECF arrangement. 
Table 1. Ghana: Selected Economic and Financial Indicators, 2008-13 ${ }^{1}$

\begin{tabular}{|c|c|c|c|c|c|c|c|}
\hline & \multirow[t]{3}{*}{2008} & \multirow[t]{3}{*}{2009} & \multirow[t]{3}{*}{2010} & \multicolumn{2}{|c|}{2011} & \multirow{3}{*}{$\begin{array}{l}2012 \\
\text { Proj. }\end{array}$} & \multirow{3}{*}{$\begin{array}{l}2013 \\
\text { Proj. }\end{array}$} \\
\hline & & & & \multicolumn{2}{|c|}{ 3/4 ECF } & & \\
\hline & & & & & Proj. & & \\
\hline & \multicolumn{7}{|c|}{ (Annual percentage change; unless otherwise specified) } \\
\hline \multicolumn{8}{|l|}{ National accounts and prices } \\
\hline Real GDP & 8.4 & 4.0 & 7.7 & 13.7 & 13.6 & 8.5 & 7.4 \\
\hline Real GDP (nonoil) & 8.4 & 4.0 & 7.7 & 6.5 & 8.2 & 7.6 & 6.3 \\
\hline Real GDP per capita & 5.7 & 1.4 & 5.0 & 10.8 & 10.8 & 5.8 & 4.8 \\
\hline GDP deflator & 20.2 & 16.6 & 17.3 & 11.2 & 9.0 & 11.9 & 7.6 \\
\hline Consumer price index (annual average) & 16.5 & 19.3 & 10.7 & 8.7 & 8.7 & 9.1 & 7.8 \\
\hline Consumer price index (end of period) & 18.1 & 16.0 & 8.6 & 9.0 & 9.0 & 8.7 & 8.0 \\
\hline \multicolumn{8}{|l|}{ External sector } \\
\hline Exports, f.o.b. (percentage change, in US\$) & 26.3 & 10.8 & 36.3 & 64.6 & 61.3 & 15.7 & 11.7 \\
\hline Excluding oil exports & 26.3 & 10.8 & 36.3 & 20.9 & 29.2 & 14.1 & 7.9 \\
\hline Imports, f.o.b. (percentage change, in US\$) & 27.3 & -21.6 & 35.7 & 33.4 & 40.5 & 10.1 & 4.2 \\
\hline Export volume & 47.5 & 13.4 & 57.4 & 10.3 & 84.1 & 27.4 & 22.6 \\
\hline Import volume & 51.6 & -36.0 & 54.6 & 16.8 & 63.7 & 10.0 & 4.1 \\
\hline Terms of trade & -9.1 & 32.6 & 3.7 & -4.0 & -2.8 & 3.7 & -1.5 \\
\hline Nominal effective exchange rate (end of period) & -11.3 & -21.7 & -0.6 & $\ldots$ & $\ldots$ & $\ldots$ & $\ldots$ \\
\hline Real effective exchange rate (end of period; depreciation -) & 1.3 & -11.2 & 6.1 & $\ldots$ & $\ldots$ & $\ldots$ & $\ldots$ \\
\hline Cedis (new) per U.S. dollar (end of period) & 1.2 & 1.4 & 1.5 & $\cdots$ & $\cdots$ & $\cdots$ & $\cdots$ \\
\hline \multicolumn{8}{|l|}{ Money and credit } \\
\hline Net domestic assets ${ }^{2}$ & 48.3 & 3.9 & 16.7 & 14.1 & 23.8 & 21.9 & 14.1 \\
\hline Credit to the private sector ${ }^{2}$ & 33.3 & 12.9 & 19.8 & 15.4 & 17.6 & 18.7 & 18.2 \\
\hline Real private sector credit (\% annual changes) & 25.4 & 0.5 & 16.4 & 13.3 & 16.4 & 19.9 & 22.5 \\
\hline Broad money (excluding foreign currency deposits) & 31.2 & 21.2 & 45.6 & 20.5 & 34.3 & 42.6 & 31.6 \\
\hline Velocity (GDP/average broad money) & 4.8 & 4.8 & 4.2 & 3.9 & 3.6 & 3.1 & 2.7 \\
\hline Prime rate (Bank of Ghana; percent; end of period) & 17.0 & 18.0 & 13.5 & $\cdots$ & $\cdots$ & $\ldots$ & $\cdots$ \\
\hline & \multicolumn{7}{|c|}{ (Percent of GDP) } \\
\hline \multicolumn{8}{|l|}{ Investment and saving } \\
\hline Gross investment & 21.5 & 20.7 & 19.7 & 20.5 & 25.8 & 26.1 & 21.4 \\
\hline Private ${ }^{3}$ & 18.5 & 15.5 & 15.8 & 18.7 & 22.4 & 21.8 & 17.6 \\
\hline Central government & 2.9 & 5.2 & 3.9 & 1.8 & 3.3 & 4.3 & 3.9 \\
\hline Gross national saving & 12.0 & 16.7 & 11.5 & 13.0 & 17.4 & 20.1 & 17.7 \\
\hline Private $^{3}$ & 9.2 & 13.4 & 9.1 & 7.5 & 11.5 & 14.6 & 11.2 \\
\hline Central government & 2.8 & 3.3 & 2.4 & 5.5 & 6.0 & 5.5 & 6.4 \\
\hline Foreign savings & 10.8 & 4.0 & 8.2 & 7.5 & 8.3 & 6.0 & 3.8 \\
\hline & \multicolumn{7}{|c|}{ (Percent of nonoil GDP) } \\
\hline \multicolumn{8}{|l|}{ Government operations } \\
\hline Total revenue & 13.3 & 13.5 & 14.4 & 18.5 & 19.3 & 19.9 & 20.2 \\
\hline Grants & 2.7 & 3.0 & 2.3 & 2.6 & 1.8 & 1.8 & 1.3 \\
\hline Total expenditure & 24.0 & 20.5 & 22.6 & 24.1 & 23.5 & 26.1 & 24.7 \\
\hline Arrears clearance and VAT refunds & 0.6 & 1.8 & 1.5 & 1.7 & 2.7 & 0.7 & 0.8 \\
\hline Overall balance (including grants) & -8.5 & -5.8 & -7.4 & -4.7 & -5.1 & -5.2 & -4.0 \\
\hline Net domestic financing & 5.7 & 2.8 & 4.6 & 3.0 & 4.4 & 2.6 & 0.4 \\
\hline Central government debt (gross) & 33.6 & 36.2 & 46.3 & 42.8 & 45.4 & 46.5 & 45.8 \\
\hline Domestic debt & 17.4 & 16.7 & 26.4 & 19.4 & 24.4 & 22.8 & 19.8 \\
\hline External debt & 16.2 & 19.5 & 19.9 & 23.4 & 21.0 & 23.7 & 26.0 \\
\hline Central government debt (net) & 30.1 & 32.7 & 43.1 & 39.6 & 42.7 & 44.1 & 43.6 \\
\hline & & (Perce & $t$ of GDP; & unless othe & wise spe & fied) & \\
\hline External sector & & & & & & & \\
\hline Current account balance (including official transfers) & -10.8 & -4.0 & -8.2 & -7.5 & -8.3 & -6.0 & -3.8 \\
\hline Current account balance (excluding official transfers) & -13.3 & -7.3 & -8.8 & -9.4 & -9.6 & -7.3 & -4.7 \\
\hline NPV of external debt outstanding & 10.2 & 10.8 & 8.4 & 17.8 & 9.7 & 13.4 & 16.3 \\
\hline percent of exports of goods and services & 41.0 & 36.8 & 28.5 & 45.1 & 25.1 & 34.9 & 41.6 \\
\hline Gross international reserves (millions of US\$) & 2,036 & 772 & 4,725 & 5,700 & 5,432 & 6,390 & 8,449 \\
\hline months of imports of goods and services & 2.2 & 0.7 & 3.0 & 3.6 & 3.1 & 3.5 & 4.3 \\
\hline Total donor support (millions of US\$) & 1,434 & 1,790 & 1,644 & 2,066 & 1,455 & 1,373 & 1,063 \\
\hline percent of GDP & 5.0 & 6.9 & 5.1 & 5.5 & 3.8 & 3.2 & 2.3 \\
\hline Memorandum items: & & & & & & & \\
\hline Nominal GDP (millions of GHc) & 30,179 & 36,599 & 46,236 & 56,226 & 57,254 & 69,538 & 80,399 \\
\hline
\end{tabular}

Sources: Data provided by Ghanaian authorities; and IMF staff estimates and projections.

${ }^{1}$ Based on new national accounts rebased to 2006, including ECF program indicators.

${ }^{2}$ Percent of broad money (including foreign currency deposits) at the beginning of the period.

${ }^{3}$ Including public enterprises and errors and omissions. 
Table 2A. Ghana: Summary of Central Government Budgetary Operations, 2008-13

\begin{tabular}{|c|c|c|c|c|c|c|c|c|}
\hline & \multirow[t]{3}{*}{2008} & \multirow[t]{3}{*}{2009} & \multirow[t]{3}{*}{2010} & \multicolumn{2}{|c|}{2011} & \multicolumn{2}{|c|}{2012} & \multirow{3}{*}{$\begin{array}{l}2013 \\
\text { Proj. }\end{array}$} \\
\hline & & & & $3 / 4 \mathrm{ECF}$ & $5 \mathrm{ECF}$ & $3 / 4 \mathrm{ECF}$ & $5 \mathrm{ECF}$ & \\
\hline & & & & May 2011 & Proj. & May 2011 & Proj. & \\
\hline & \multicolumn{7}{|c|}{ (Percent of nonoil GDP, unless otherwise specified) } & \\
\hline Total revenue and grants & 16.0 & 16.5 & 16.7 & 21.1 & 21.1 & 21.5 & 21.7 & 21.5 \\
\hline Revenue & 13.3 & 13.5 & 14.4 & 18.5 & 19.3 & 19.8 & 19.9 & 20.2 \\
\hline Oil revenue & $\ldots$ & $\ldots$ & $\ldots$ & 2.4 & 1.2 & 3.6 & 1.9 & 2.7 \\
\hline Nonoil revenue & 13.3 & 13.5 & 14.4 & 16.1 & 18.0 & 16.1 & 18.0 & 17.6 \\
\hline Tax revenue & 12.9 & 12.3 & 13.2 & 14.9 & 16.7 & 15.6 & 17.5 & 17.0 \\
\hline Direct taxes & 4.2 & 4.7 & 5.3 & 6.1 & 7.0 & 6.3 & 7.7 & 7.5 \\
\hline Indirect taxes & 6.4 & 5.5 & 5.4 & 6.1 & 6.7 & 6.5 & 6.7 & 6.6 \\
\hline Trade taxes & 2.4 & 2.1 & 2.5 & 2.7 & 3.0 & 2.8 & 3.0 & 2.9 \\
\hline Nontax revenue & 0.4 & 1.3 & 1.2 & 1.1 & 1.3 & 0.5 & 0.5 & 0.5 \\
\hline Grants & 2.7 & 3.0 & 2.3 & 2.6 & 1.8 & 1.7 & 1.8 & 1.3 \\
\hline Total expenditure & 24.0 & 20.5 & 22.6 & 24.1 & 23.0 & 22.9 & 24.6 & 24.7 \\
\hline Recurrent expenditure & 14.8 & 13.4 & 15.1 & 16.0 & 16.1 & 15.6 & 15.8 & 16.7 \\
\hline Recurrent Non-interest expenditure & 12.6 & 10.6 & 12.0 & 12.7 & 13.0 & 12.4 & 12.9 & 13.3 \\
\hline Wages and salaries ${ }^{1}$ & 6.6 & 6.8 & 6.9 & 7.6 & 8.0 & 7.3 & 7.8 & 8.1 \\
\hline Goods and services & 2.1 & 1.7 & 2.1 & 1.8 & 1.5 & 1.9 & 1.5 & 1.9 \\
\hline Transfers & 2.9 & 1.6 & 2.0 & 2.5 & 2.7 & 2.4 & 2.6 & 2.4 \\
\hline Reserve Fund & 0.9 & 0.5 & 1.0 & 0.8 & 0.8 & 0.8 & 1.1 & 0.8 \\
\hline Interest costs & 2.3 & 2.8 & 3.1 & 3.3 & 3.2 & 3.2 & 2.9 & 3.4 \\
\hline domestic & 1.6 & 2.1 & 2.4 & 2.7 & 2.6 & 2.3 & 2.1 & 2.5 \\
\hline foreign & 0.7 & 0.7 & 0.7 & 0.6 & 0.6 & 1.0 & 0.8 & 0.9 \\
\hline Capital expenditure (total) & 9.1 & 7.1 & 7.5 & 8.2 & 6.8 & 7.3 & 8.8 & 8.0 \\
\hline Domestic & 6.1 & 2.7 & 3.1 & 3.8 & 4.1 & 4.8 & 4.1 & 4.3 \\
\hline Foreign & 3.0 & 4.4 & 4.4 & 4.3 & 2.8 & 2.4 & 4.7 & 3.8 \\
\hline Arrears clearance ${ }^{2}$ & -0.5 & -1.7 & -1.4 & -1.6 & -2.6 & -1.0 & -0.6 & -0.8 \\
\hline Deferred wage payments & $\ldots$ & $\ldots$ & $\ldots$ & $\ldots$ & -0.5 & $\ldots$ & -1.5 & 0.0 \\
\hline VAT refunds & -0.1 & -0.1 & -0.1 & -0.1 & -0.1 & -0.1 & -0.1 & -0.1 \\
\hline Overall balance (after arrears) & -8.5 & -5.8 & -7.4 & -4.7 & -5.1 & -2.5 & -5.2 & -4.2 \\
\hline Discrepancy & 0.4 & -0.2 & -0.9 & 0.9 & 0.0 & 0.0 & 0.0 & 0.0 \\
\hline Total financing & 8.9 & 5.6 & 6.5 & 5.7 & 5.1 & 2.5 & 5.2 & 4.2 \\
\hline Divestiture receipts (net) & 2.3 & 0.0 & -1.0 & -1.1 & -1.1 & 0.0 & 0.0 & 0.0 \\
\hline Foreign (net) & 0.6 & 2.6 & 2.6 & 3.5 & 1.5 & 2.6 & 2.4 & 3.4 \\
\hline Exceptional financing (debt relief, bilateral) & 0.3 & 0.2 & 0.2 & 0.2 & 0.2 & 0.2 & 0.2 & 0.2 \\
\hline Domestic (net) & 5.7 & 2.8 & 4.6 & 3.0 & 4.4 & -0.3 & 2.6 & 0.6 \\
\hline Banking system & 4.2 & 2.1 & 2.1 & 1.4 & 2.4 & -1.8 & 1.5 & 0.2 \\
\hline Non-bank & 1.6 & 0.8 & 2.5 & 1.6 & 2.0 & 1.6 & 1.1 & 0.4 \\
\hline of which: non resident purchase of $\mathrm{T}$ bills & 1.5 & 0.1 & 2.3 & 1.2 & 1.6 & 1.1 & 0.8 & 0.2 \\
\hline \multicolumn{9}{|l|}{ Memorandum items: } \\
\hline Poverty-reducing expenditures & 5.2 & 5.1 & 5.2 & 5.4 & 5.4 & 5.4 & 5.3 & 5.4 \\
\hline \multicolumn{9}{|l|}{ Domestic arrears } \\
\hline New arrears & 4.5 & 2.4 & 2.6 & 0.3 & 0.0 & 0.0 & 0.0 & 0.0 \\
\hline Arrears cleared ${ }^{3}$ & 0.5 & 1.7 & 1.4 & 2.5 & 2.6 & 1.0 & 0.2 & 0.8 \\
\hline Arrears outstanding ${ }^{4}$ & 4.5 & 4.4 & 4.7 & 2.4 & 1.4 & 1.1 & 0.9 & 0.0 \\
\hline Budget balance (commitment basis) & -12.5 & -6.5 & -9.8 & -3.4 & -3.7 & -1.5 & -3.0 & -3.3 \\
\hline Total government debt (gross) & 33.6 & 36.2 & 46.3 & 42.8 & 45.4 & 42.4 & 46.4 & 45.6 \\
\hline Domestic debt & 17.4 & 16.7 & 26.4 & 19.4 & 24.4 & 18.7 & 22.7 & 19.6 \\
\hline External debt & 16.2 & 19.5 & 19.9 & 23.4 & 21.0 & 23.6 & 23.7 & 26.0 \\
\hline Total government debt (net) & 30.1 & 32.7 & 43.1 & 39.6 & 42.7 & 37.2 & 44.0 & 43.5 \\
\hline Nominal nonoil GDP (millions of GHc) & 30,179 & 36,599 & 46,236 & 51,567 & 53,775 & 59,993 & 65,034 & 74,338 \\
\hline
\end{tabular}

Sources: Ghanaian authorities; and IMF staff estimates and projections.

${ }^{1}$ Excludes deferred wage payments

${ }^{2}$ For 2010, excludes payment of outstanding floats. In 2012, includes reserves to cover expected float payments from 2011 commitments. This reserve is included in expenditures (commitment basis) for 2011.

${ }^{3}$ For 2010, excludes payment of outstanding floats. In 2012, excludes reserves to cover expected float payments from 2011 commitments.

${ }^{4}$ Excludes SOEs' liabilities and deferred wage payments. 
Table 2B. Ghana: Summary of Central Government Budgetary Operations, 2008-13

\begin{tabular}{|c|c|c|c|c|c|c|c|c|}
\hline & \multirow{3}{*}{2008} & \multirow[t]{3}{*}{2009} & \multirow[t]{3}{*}{2010} & \multicolumn{2}{|c|}{2011} & \multicolumn{2}{|c|}{2012} & \multirow{3}{*}{$\begin{array}{r}2013 \\
\text { Proj. }\end{array}$} \\
\hline & & & & $3 / 4 \mathrm{ECF}$ & $5 \mathrm{ECF}$ & 3/4 ECF & $5 \mathrm{ECF}$ & \\
\hline & & & & May 2011 & Proj. & May 2011 & Proj. & \\
\hline & \multicolumn{7}{|c|}{ (Millions of GHc, unless otherwise specified) } & \\
\hline Total revenue and grants & 4,839 & 6,048 & 7,739 & 10,875 & 11,348 & 12,907 & 14,091 & 15,983 \\
\hline Revenue & 4,019 & 4,947 & 6,659 & 9,529 & 10,358 & 11,865 & 12,935 & 15,024 \\
\hline Oil revenue & $\ldots$ & $\ldots$ & $\ldots$ & 1,251 & 652 & 2,188 & 1,240 & 1,977 \\
\hline Nonoil revenue & 4,019 & 4,947 & 6,659 & 8,278 & 9,706 & 9,678 & 11,695 & 13,047 \\
\hline Tax revenue & 3,893 & 4,485 & 6,118 & 7,697 & 8,997 & 9,355 & 11,362 & 12,644 \\
\hline Direct taxes & 1,253 & 1,717 & 2,454 & 3,130 & 3,769 & 3,767 & 5,035 & 5,585 \\
\hline Indirect taxes & 1,920 & 2,006 & 2,518 & 3,150 & 3,597 & 3,879 & 4,354 & 4,917 \\
\hline Trade taxes & 719 & 763 & 1,146 & 1,417 & 1,631 & 1,709 & 1,973 & 2,142 \\
\hline Nontax revenue & 126 & 461 & 541 & 582 & 709 & 322 & 332 & 402 \\
\hline Grants & 821 & 1,101 & 1,080 & 1,347 & 989 & 1,042 & 1,156 & 959 \\
\hline Total expenditure & 7,229 & 7,521 & 10,461 & 12,442 & 12,360 & 13,730 & 15,992 & 18,368 \\
\hline Recurrent expenditure & 4,469 & 4,904 & 6,974 & 8,228 & 8,678 & 9,377 & 10,294 & 12,403 \\
\hline Recurrent Non-interest expenditure & 3,789 & 3,872 & 5,535 & 6,540 & 6,981 & 7,430 & 8,410 & 9,855 \\
\hline Wages and salaries ${ }^{1}$ & 1,988 & 2,479 & 3,183 & 3,910 & 4,324 & 4,394 & 5,050 & 6,054 \\
\hline Goods and services & 648 & 621 & 962 & 952 & 786 & 1,128 & 967 & 1,400 \\
\hline Transfers & 878 & 604 & 920 & 1,274 & 1,467 & 1,431 & 1,685 & 1,809 \\
\hline Reserve Fund & 275 & 168 & 471 & 404 & 404 & 478 & 708 & 592 \\
\hline Interest costs & 679 & 1,032 & 1,439 & 1,688 & 1,697 & 1,946 & 1,884 & 2,548 \\
\hline domestic & 482 & 774 & 1,124 & 1,372 & 1,372 & 1,372 & 1,387 & 1,893 \\
\hline foreign & 197 & 259 & 315 & 316 & 325 & 574 & 496 & 655 \\
\hline Capital expenditure (total) & 2,760 & 2,616 & 3,486 & 4,214 & 3,682 & 4,354 & 5,698 & 5,965 \\
\hline Domestic & 1,844 & 990 & 1,454 & 1,981 & 2,202 & 2,887 & 2,666 & 3,174 \\
\hline Foreign & 916 & 1,627 & 2,033 & 2,233 & 1,480 & 1,467 & 3,032 & 2,791 \\
\hline Overall balance (before arrears) & $-2,415$ & $-1,504$ & $-2,767$ & $-1,620$ & $-1,066$ & -891 & $-1,978$ & $-2,470$ \\
\hline Arrears clearance ${ }^{2}$ & -142 & -626 & -642 & -809 & $-1,412$ & -600 & -400 & -616 \\
\hline Deferred wage payments & $\ldots$ & $\ldots$ & $\ldots$ & $\ldots$ & -263 & $\ldots$ & -991 & 0 \\
\hline VAT refunds & -26 & -31 & -45 & -53 & -53 & -68 & -77 & -86 \\
\hline Overall balance (after arrears) & $-2,558$ & $-2,131$ & $-3,408$ & $-2,429$ & $-2,741$ & $-1,490$ & $-3,369$ & $-3,087$ \\
\hline Discrepancy & 133 & -69 & -409 & 489 & 0 & 0 & 0 & 0 \\
\hline Total financing & 2,690 & 2,062 & 3,000 & 2,918 & 2,741 & 1,490 & 3,369 & 3,087 \\
\hline Divestiture receipts (net) & 707 & 6 & -445 & -572 & -572 & 0 & 0 & 0 \\
\hline Foreign (net) & 171 & 955 & 1,209 & 1,824 & 828 & 1,538 & 1,572 & 2,542 \\
\hline Exceptional financing (debt relief, bilateral) & 78 & 59 & 93 & 122 & 123 & 119 & 131 & 118 \\
\hline Domestic (net) & 1,735 & 1,042 & 2,143 & 1,544 & 2,361 & -167 & 1,666 & 427 \\
\hline Banking system & 1,255 & 767 & 973 & 744 & 1,286 & $-1,098$ & 951 & 130 \\
\hline Non-bank & 479 & 275 & 1,170 & 800 & 1,076 & 931 & 715 & 297 \\
\hline of which: non resident purchase of T bills & 439 & 44 & 1,081 & 640 & 860 & 651 & 501 & 178 \\
\hline \multicolumn{9}{|l|}{ Memorandum items: } \\
\hline Nominal nonoil GDP (millions of GHc) & 30,179 & 36,599 & 46,236 & 51,567 & 53,775 & 59,993 & 65,034 & 74,338 \\
\hline Poverty-reducing expenditures & 1,584 & 1,856 & 2,411 & 2,797 & 2,883 & 3,254 & 3,434 & 4,049 \\
\hline \multicolumn{9}{|l|}{ Domestic arrears } \\
\hline New arrears & 1,353 & 866 & 1,208 & 132 & 0 & 0 & 0 & 0 \\
\hline Arrears cleared ${ }^{3}$ & 142 & 627 & 642 & 1,298 & 1,412 & 600 & 130 & 616 \\
\hline Arrears outstanding ${ }^{4}$ & 1,353 & 1,592 & 2,158 & 1,254 & 747 & 655 & 616 & 0 \\
\hline Budget balance (commitment basis) & $-3,768$ & $-2,370$ & $-4,552$ & $-1,752$ & $-2,012$ & -891 & $-1,978$ & $-2,470$ \\
\hline Total government debt (gross) & 10,138 & 13,256 & 21,428 & 22,071 & 24,392 & 25,416 & 30,189 & 33,902 \\
\hline Domestic debt & 5,239 & 6,103 & 12,210 & 10,007 & 13,095 & 11,248 & 14,763 & 14,560 \\
\hline External debt & 4,899 & 7,153 & 9,218 & 12,064 & 11,298 & 14,168 & 15,425 & 19,343 \\
\hline Total government debt (net) & 9,083 & 11,963 & 19,942 & 20,403 & 22,971 & 22,340 & 28,635 & 32,363 \\
\hline
\end{tabular}

Sources: Ghanaian authorities; and IMF staff estimates and projections.

${ }^{1}$ Excludes deferred wage payments

${ }^{2}$ For 2010, excludes payment of outstanding floats. In 2012, includes reserves to cover expected float payments from 2011 commitments. This reserve is included in expenditures (commitment basis) for 2011.

${ }^{3}$ For 2010, excludes payment of outstanding floats. In 2012, excludes reserves to cover expected float payments from 2011 commitments.

${ }^{4}$ Excludes SOEs' liabilities and deferred wage payments. 
Table 3. Ghana: Monetary Survey, 2009-13

(Millions of cedis, unless otherwise indicated)

\begin{tabular}{|c|c|c|c|c|c|c|c|c|c|c|c|c|c|c|}
\hline & \multirow[t]{4}{*}{2009} & \multirow[t]{4}{*}{2010} & \multicolumn{7}{|c|}{2011} & \multicolumn{4}{|c|}{2012} & \multirow{4}{*}{$\begin{array}{l}2013 \\
\text { Proj }\end{array}$} \\
\hline & & & \multirow{2}{*}{\multicolumn{3}{|c|}{ Mar $\frac{\text { Jun }}{3 / 4 \mathrm{ECF}}$}} & \multicolumn{2}{|c|}{ Sep } & \multicolumn{2}{|c|}{ Dec } & \multirow[t]{2}{*}{ Mar } & \multirow[t]{2}{*}{ Jun } & \multirow[t]{2}{*}{ Sep } & \multirow[t]{2}{*}{ Dec } & \\
\hline & & & & & & $3 / 4 \mathrm{ECF}$ & & 3/4 ECF & & & & & & \\
\hline & & & Act. & Prog. & Prel. & Prog. & Est. & Prog. & Proj. & Proj. & Proj. & Proj. & Proj. & \\
\hline \multicolumn{15}{|l|}{ Bank of Ghana } \\
\hline Net foreign assets & 3,261 & 5,241 & 5,237 & 4,831 & 5,485 & 4,841 & 5,311 & 6,568 & 6,263 & 6,205 & 6,079 & 6,443 & 8,025 & 12,176 \\
\hline (in millions of U.S. dollars) & 2,273 & 3,556 & 3,485 & 3,221 & 3,637 & 3,227 & 3,481 & 4,379 & 4,074 & 3,959 & 3,805 & 3,959 & 4,842 & 6,901 \\
\hline Net domestic assets, of which: & -229 & -831 & -924 & -469 & $-1,242$ & -329 & -970 & $-1,252$ & -341 & 175 & 505 & 312 & 421 & $-1,058$ \\
\hline Claims on government (net) & 1,355 & 1,371 & 1,214 & 1,374 & 589 & 1,194 & 1,161 & 1,014 & 151 & 31 & -88 & -164 & $-1,331$ & $-2,742$ \\
\hline Other items, net (assets + ) & -799 & -926 & $-1,117$ & -900 & $-1,103$ & -900 & $-1,289$ & -900 & $-1,228$ & $-1,109$ & -995 & -876 & -647 & -647 \\
\hline Reserve money (RM), of which: & 3,032 & 4,410 & 4,313 & 4,362 & 4,243 & 4,512 & 4,341 & 5,317 & 5,922 & 6,380 & 6,583 & 6,755 & 8,447 & 11,118 \\
\hline Currency outside banks & 2,084 & 2,927 & 2,606 & 2,686 & 2,786 & 2,796 & 3,154 & 3,481 & 3,522 & 3,230 & 3,765 & 3,656 & 4,365 & 5,120 \\
\hline Bank reserves & 872 & 1,320 & 1,435 & 1,503 & 1,327 & 1,543 & 1,813 & 1,663 & 2,300 & 3,049 & 2,717 & 2,998 & 3,981 & 5,897 \\
\hline \multicolumn{15}{|l|}{ Monetary survey } \\
\hline Net foreign assets & 3,925 & 5,754 & 6,223 & 5,205 & 6,792 & 5,174 & 6,007 & 6,861 & 7,988 & 8,145 & 8,656 & 9,388 & 11,339 & 16,054 \\
\hline (in millions of U.S. dollars) & 2,733 & 3,915 & 4,142 & 3,470 & 4,509 & 3,450 & 4,478 & 4,574 & 5,196 & 5,129 & 5,360 & 5,736 & 6,841 & 9,100 \\
\hline Net domestic assets & 6,308 & 8,012 & 8,245 & 8,646 & 8,410 & 9,155 & 9,765 & 9,919 & 11,287 & 12,011 & 12,491 & 12,533 & 15,501 & 19,287 \\
\hline Domestic credit & 10,149 & 12,878 & 12,763 & 13,767 & 13,094 & 14,166 & 13,943 & 14,750 & 16,559 & 17,184 & 17,200 & 17,059 & 20,431 & 24,452 \\
\hline Claims on government (net) & 3,169 & 4,352 & 3,976 & 4,635 & 4,090 & 4,821 & 4,411 & 5,007 & 5,638 & 4,019 & 4,965 & 4,527 & 6,588 & 6,618 \\
\hline Claims on non-government & 6,979 & 8,526 & 8,787 & 9,133 & 9,005 & 9,345 & 9,532 & 9,744 & 10,921 & 13,166 & 12,235 & 12,531 & 13,843 & 17,834 \\
\hline Other items, net (assets + ) & $-3,860$ & $-4,898$ & $-4,553$ & $-4,833$ & $-4,722$ & $-4,832$ & $-4,487$ & $-4,832$ & $-5,271$ & $-5,174$ & $-4,709$ & $-4,526$ & $-4,930$ & $-5,165$ \\
\hline Broad money (M2+) & 10,233 & 13,766 & 14,468 & 13,851 & 15,202 & 14,329 & 15,772 & 16,780 & 19,276 & 20,155 & 21,147 & 21,921 & 26,839 & 35,341 \\
\hline Currency & 2,084 & 2,927 & 2,606 & 2,397 & 2,786 & 2,617 & 3,154 & 3,481 & 3,522 & 3,230 & 3,765 & 3,656 & 4,365 & 5,120 \\
\hline Deposits & 8,149 & 10,839 & 11,862 & 11,454 & 12,416 & 11,712 & 12,618 & 13,299 & 15,754 & 16,925 & 17,382 & 18,264 & 22,475 & 30,221 \\
\hline Domestic currency deposits & 5,484 & 8,090 & 8,571 & 8,289 & 8,746 & 8,437 & 8,679 & 9,801 & 11,275 & 12,711 & 12,683 & 13,220 & 16,740 & 22,658 \\
\hline Foreign currency deposits & 2,664 & 2,749 & 3,291 & 3,165 & 3,670 & 3,276 & 3,939 & 3,497 & 4,479 & 4,215 & 4,699 & 5,044 & 5,735 & 7,562 \\
\hline (in millions of U.S. dollars) & 1,865 & 1,865 & 2,191 & 2,138 & 2,436 & 2,213 & 2,644 & 2,332 & 2,913 & 2,543 & 2,835 & 3,044 & 3,460 & 4,286 \\
\hline Broad money (M2) ${ }^{1}$ & 7,569 & 11,017 & 11,177 & 10,686 & 11,532 & 11,054 & 11,832 & 13,282 & 14,797 & 15,941 & 16,448 & 16,876 & 21,104 & 27,778 \\
\hline Memorandum items: & \multicolumn{13}{|c|}{ (Annual percentage change, unless otherwise indicated) } & \\
\hline Broad money (M2) & 21.2 & 45.6 & 36.6 & 25.1 & 35.0 & 25.2 & 34.1 & 20.5 & 34.3 & 48.4 & 54.8 & 51.0 & 42.6 & 31.6 \\
\hline Broad money (M2+) & 26.9 & 34.5 & 35.5 & 25.0 & 37.2 & 25.0 & 37.6 & 21.8 & 40.0 & 46.6 & 53.2 & 51.5 & 39.2 & 31.7 \\
\hline Reserve money (RM) & 36.3 & 45.4 & 37.6 & 56.8 & 52.6 & 43.3 & 37.9 & 20.5 & 34.3 & 50.5 & 61.2 & 56.6 & 42.6 & 31.6 \\
\hline Bank of Ghana NDA; at program exchange rate ${ }^{2}$ & -264 & -924.2 & -914 & -469 & -1212 & -329 & -880 & $-1,252$ & -341 & 294 & 733 & 668 & 1,002 & 766 \\
\hline Velocity (non-oil GDP/end-of-period M2) & 4.8 & 4.2 & & & & & & 3.9 & 3.6 & & & & 3.1 & 2.7 \\
\hline Reserve money multiplier (M2/RM) & 2.5 & 2.5 & 2.6 & 2.5 & 2.7 & 2.5 & 2.7 & 2.5 & 2.5 & 2.5 & 2.5 & 2.5 & 2.5 & 2.5 \\
\hline Foreign currency deposits to total deposits & 32.7 & 25.4 & 27.7 & 27.6 & 29.6 & 28.0 & 31.2 & 26.3 & 28.4 & 24.9 & 27.0 & 27.6 & 25.5 & 25.0 \\
\hline Currency-to-deposits & 25.6 & 27.0 & 22.0 & 23.4 & 22.4 & 23.9 & 25.0 & 26.2 & 22.4 & 19.1 & 21.7 & 20.0 & 19.4 & 16.9 \\
\hline Credit to the private sector & 16.5 & 27.5 & 24.5 & 35.0 & 30.4 & 35.1 & 22.6 & 23.6 & 25.8 & 52.8 & 28.8 & 37.6 & 30.4 & 32.3 \\
\hline Real credit to private sector (\% changes) ${ }^{3}$ & 0.5 & 17.4 & 14.1 & 23.9 & 19.6 & 24.3 & 12.8 & 13.3 & 15.4 & 18.3 & 25.4 & 16.5 & 19.9 & 22.5 \\
\hline
\end{tabular}

Sources: Bank of Ghana, and IMF staff estimates and projections.

${ }^{1}$ Excluding foreign currency deposits.

2 In millions of cedis. The program exchange rate is GHc 1.50 per U.S. for January-September 2011, and GHc 1.5374 for Dec 2011 and 2012

${ }^{3}$ Deflated by the Consumer Price Index. 
Table 4. Ghana: Balance of Payments, 2008-13

\begin{tabular}{|c|c|c|c|c|c|c|c|}
\hline & \multirow[b]{2}{*}{2008} & \multirow[b]{2}{*}{2009} & \multirow[b]{2}{*}{2010} & \multicolumn{2}{|c|}{2011} & \multirow{2}{*}{$\begin{array}{l}2012 \\
\text { Proj. }\end{array}$} & \multirow{2}{*}{$\begin{array}{l}2013 \\
\text { Proj. }\end{array}$} \\
\hline & & & & $\begin{array}{r}/ 4 \text { ECF } \\
\text { Prog. }\end{array}$ & Proj. & & \\
\hline & \multicolumn{5}{|c|}{ (Millions of US dollars) } & & \\
\hline Balance on Current Account (including grants) & $-3,080$ & $-1,035$ & $-2,659$ & $-2,804$ & $-3,159$ & $-2,614$ & $-1,780$ \\
\hline Trade balance & $-4,999$ & $-2,207$ & $-2,962$ & $-1,284$ & $-2,508$ & $-2,046$ & $-1,005$ \\
\hline Exports f.o.b. & 5,270 & 5,840 & 7,960 & 12,997 & 12,841 & 14,857 & 16,602 \\
\hline Cocoa & 1,487 & 1,866 & 2,220 & 2,531 & 2,619 & 2,606 & 2,751 \\
\hline Gold & 2,246 & 2,551 & 3,804 & 5,001 & 5,307 & 6,656 & 7,328 \\
\hline Oil & 0 & 0 & 0 & 3,450 & 2,558 & 3,123 & 3,936 \\
\hline Other & 1,536 & 1,422 & 1,937 & 2,015 & 2,356 & 2,472 & 2,587 \\
\hline Imports, f.o.b. & $-10,268$ & $-8,046$ & $-10,922$ & $-14,281$ & $-15,348$ & $-16,902$ & $-17,607$ \\
\hline Non-oil & $-7,912$ & $-6,557$ & $-8,686$ & $-11,415$ & $-12,136$ & $-13,517$ & $-14,218$ \\
\hline Oil & $-2,357$ & $-1,489$ & $-2,236$ & $-2,866$ & $-3,212$ & $-3,386$ & $-3,389$ \\
\hline Balance on services and income & -756 & $-1,470$ & $-2,019$ & $-4,218$ & $-3,038$ & $-3,578$ & $-3,734$ \\
\hline Of which: interest payments & -172 & -157 & -192 & -199 & -138 & -169 & -263 \\
\hline Balance on transfers & 2,675 & 2,642 & 2,322 & 2,698 & 2,387 & 3,010 & 2,960 \\
\hline Private transfers (net) & 1,970 & 1,788 & 2,123 & 1,963 & 1,895 & 2,461 & 2,554 \\
\hline Official transfers (net) & 704 & 854 & 199 & 735 & 492 & 548 & 406 \\
\hline Capital and Financial Account & 2,992 & 3,115 & 4,290 & 3,377 & 3,724 & 3,383 & 3,839 \\
\hline Capital account (net) & 463 & 564 & 337 & 0 & 320 & 270 & 270 \\
\hline Financial Account (net) & 2,529 & 2,551 & 3,952 & 3,377 & 3,404 & 3,113 & 3,569 \\
\hline Official financing (medium and long-term loans) & 487 & 1,086 & 880 & 1,707 & 910 & 1,642 & 1,657 \\
\hline Inflows & 718 & 1,246 & 1,058 & 1,931 & 1,132 & 2,034 & 1,920 \\
\hline Amortization & -231 & -160 & -178 & -224 & -222 & -392 & -263 \\
\hline Private capital & 2,307 & 1,499 & 2,654 & 1,670 & 2,805 & 2,847 & 2,112 \\
\hline Direct investment & 1,212 & 1,678 & 2,527 & $\cdots$ & 3,122 & 3,188 & 1,688 \\
\hline Divestiture & 900 & 0 & 0 & 0 & 0 & 0 & 0 \\
\hline Portfolio & 0 & -44 & 620 & $\cdots$ & 234 & 210 & 225 \\
\hline Other & 196 & -135 & -493 & $\ldots$ & -551 & -551 & 200 \\
\hline Short-term capital & -265 & -34 & 418 & $\ldots$ & -312 & $-1,376$ & -200 \\
\hline Errors and omissions & -853 & $-3,030$ & -173 & 0 & -47 & 0 & 0 \\
\hline Overall balance & -941 & -950 & 1,458 & 573 & 518 & 768 & 2,059 \\
\hline Financing & 941 & 950 & $-1,458$ & -573 & -518 & -768 & $-2,059$ \\
\hline Net international reserves (-, incr.) & 941 & 1,369 & $-1,458$ & -788 & -518 & -768 & $-2,059$ \\
\hline Increase in gross reserves (-) & 941 & 1,264 & $-1,583$ & -975 & -708 & -958 & $-2,059$ \\
\hline Use of Fund credit & 0 & 104 & 124 & 187 & 189 & 190 & 0 \\
\hline \multicolumn{8}{|l|}{ Memorandum items: } \\
\hline Current account bal., incl. official transfers (in percent of GDP) & -10.8 & -4.0 & -8.2 & -7.5 & -8.3 & -6.0 & -3.8 \\
\hline Current account bal., excl. official transfers (in percent of GDP) & -13.3 & -7.3 & -8.8 & -9.4 & -9.6 & -7.3 & -4.7 \\
\hline Trade balance (in percent of GDP) & -17.5 & -8.6 & -9.2 & -3.4 & -6.6 & -4.7 & -2.1 \\
\hline \multicolumn{8}{|l|}{ Gross international reserves ${ }^{1}$} \\
\hline In millions of US dollars & 2,036 & 3,301 & 4,725 & 5,700 & 5,432 & 6,390 & 8,449 \\
\hline In months of prospective imports of goods and services & 2.2 & 2.8 & 3.0 & 3.6 & 3.1 & 3.5 & 4.3 \\
\hline External debt (percent of GDP) & 14.1 & 19.4 & 19.4 & 21.5 & 19.4 & 21.4 & 23.3 \\
\hline External debt service paid (in percent of exports of GNFS) & 5.1 & 4.6 & 3.9 & 2.9 & 2.5 & 3.4 & 2.9 \\
\hline Total donor support (percent of GDP) & 5.3 & 7.2 & 4.1 & 6.2 & 4.0 & 3.2 & 2.4 \\
\hline of which, official transfers (percent of GDP) & 2.5 & 3.3 & 0.6 & 2.0 & 1.3 & 1.3 & 0.9 \\
\hline Terms of trade $(1990=100)$ & 83 & 110 & 114 & 113 & 111 & 115 & 114 \\
\hline Change in the Terms of Trade & -9.1 & 32.6 & 3.7 & -4.0 & -2.8 & 3.7 & -1.5 \\
\hline GDP (US\$) & 28,528 & 25,799 & 32,321 & 37,481 & 37,879 & 43,396 & 46,994 \\
\hline
\end{tabular}

Sources: Bank of Ghana; and IMF staff estimates and projections.

${ }^{1}$ Reserves are evaluated at market exchange rates. 
Table 5. Ghana: Financial Soundness Indicators, 2005-11

(Percent, end-of-period, unless otherwise specified)

\begin{tabular}{|c|c|c|c|c|c|c|c|}
\hline & 2005 & 2006 & 2007 & 2008 & 2009 & 2010 & $\begin{array}{r}2011 \\
\text { Aug }\end{array}$ \\
\hline \multicolumn{8}{|l|}{ Capital adequacy: } \\
\hline Regulatory capital ratio & 16.2 & 15.8 & 14.8 & 13.8 & 18.2 & 19.1 & 16.9 \\
\hline Regulatory tier 1 capital ratio & 7.2 & 15.0 & 13.6 & 12.8 & 17.0 & 18.6 & 15.4 \\
\hline \multicolumn{8}{|l|}{ Asset quality: } \\
\hline Nonperforming loans to total gross loans & 13.0 & 7.9 & 6.4 & 7.7 & 16.2 & 17.6 & 16.0 \\
\hline Credit to total assets & 48.7 & 45.0 & 50.3 & 52.3 & 43.8 & 40.1 & 36.5 \\
\hline Loan provision to Gross loan & 8.5 & 7.4 & 5.5 & 6.3 & 11.1 & 9.4 & 8.6 \\
\hline Bank Provisions to NPLs & $\ldots$ & $\ldots$ & $\ldots$ & $\ldots$ & 68.7 & 70.6 & 74.9 \\
\hline \multicolumn{8}{|l|}{ Earnings and profitability: } \\
\hline Return on assets, before taxes (average) & 4.6 & 4.8 & 3.7 & 3.2 & 2.8 & 3.8 & 3.5 \\
\hline Return on equity, before taxes (average) & 23.6 & 39.6 & 35.8 & 23.7 & 17.5 & 20.4 & 17.7 \\
\hline Interest margin to gross income & 64.0 & 51.8 & 46.1 & 41.3 & 39.4 & 50.1 & 48.1 \\
\hline Interest spread ${ }^{1}$ & 16.7 & 17.7 & 17.0 & 13.8 & 17.8 & 18.7 & 19.8 \\
\hline \multicolumn{8}{|l|}{ Liquidity: } \\
\hline Core liquid assets to total assets ratio & 20.7 & 23.5 & 23.4 & 25.2 & 26.3 & 25.3 & 25.1 \\
\hline Broad liquid assets to total assets ratio & 47.0 & 46.3 & 40.7 & 39.4 & 47.2 & 51.3 & 54.9 \\
\hline Core liquid assets to short-term liabilities ratio & 42.8 & 31.4 & 31.0 & 33.5 & 34.5 & 32.9 & 32.0 \\
\hline Broad liquid assets to short-term liabilities ratio & 97.4 & 61.9 & 54.1 & 52.4 & 62.0 & 66.6 & 70.1 \\
\hline \multicolumn{8}{|l|}{ Exposure to foreign exchange risk: } \\
\hline Share of foreign currency deposits in total deposits & 26.3 & 28.1 & 22.3 & 28.4 & 32.7 & 25.4 & 29.4 \\
\hline Share of foreign liabilities in total liabilities & 2.4 & 4.0 & 8.1 & 7.0 & 6.2 & 4.7 & 3.8 \\
\hline
\end{tabular}

Source: Bank of Ghana.

${ }^{1}$ Average lending rate minus average (saving and demand) deposit rate. 
Table 6. Ghana: External Financing Requirements and Sources, 2009-16 (Millions of U.S. dollars)

\begin{tabular}{|c|c|c|c|c|c|c|c|c|}
\hline & \multirow[b]{2}{*}{2009} & \multirow[b]{2}{*}{2010} & \multicolumn{6}{|c|}{ IMF Staff Projections } \\
\hline & & & 2011 & 2012 & 2013 & 2014 & 2015 & 2016 \\
\hline Total requirements & $-3,312$ & $-4,619$ & $-4,580$ & $-4,512$ & $-4,508$ & $-5,237$ & $-5,318$ & $-5,421$ \\
\hline Current account (excl. official transfers) & $-1,888$ & $-2,858$ & $-3,651$ & $-3,163$ & $-2,186$ & $-2,755$ & $-3,064$ & $-3,057$ \\
\hline Debt amortization & -160 & -178 & -222 & -392 & -263 & -329 & -399 & -450 \\
\hline Gross reserves accumulation & $-1,264$ & $-1,583$ & -708 & -958 & $-2,059$ & $-2,153$ & $-1,854$ & $-1,914$ \\
\hline Total sources & 2,652 & 5,053 & 4,580 & 4,512 & 4,508 & 5,237 & 5,318 & 5,422 \\
\hline IMF & 104 & 124 & 189 & 190 & 0 & 0 & 0 & 0 \\
\hline Other official flows & 1,790 & 1,645 & 1,624 & 2,582 & 2,325 & 2,461 & 2,422 & 2,362 \\
\hline Foreign direct investment (net) & 1,678 & 2,527 & 3,122 & 3,188 & 1,688 & 1,843 & 2,018 & 2,188 \\
\hline Other flows ${ }^{1}$ & -921 & 756 & -355 & $-1,447$ & 495 & 934 & 878 & 872 \\
\hline
\end{tabular}

Sources: Ghanaian authorities and IMF staff estimates and projections.

${ }^{1}$ Includes all other net financial flows and errors and omissions. 
Table 7. Ghana: Indicators of Capacity to Repay the Fund, 2011-2022

\begin{tabular}{|c|c|c|c|c|c|c|c|c|c|c|c|c|}
\hline & 2011 & 2012 & 2013 & 2014 & 2015 & 2016 & 2017 & 2018 & 2019 & 2020 & 2021 & 2022 \\
\hline & \multicolumn{12}{|c|}{ Projections } \\
\hline \multicolumn{13}{|c|}{ Fund obligations based on existing credit (in millions of SDRs) } \\
\hline Principal & 10.5 & 21.1 & 21.1 & 21.1 & 42.8 & 46.3 & 41.8 & 41.8 & 41.8 & 20.1 & 6.0 & 0.0 \\
\hline Charges and interest & 0.1 & 1.0 & 0.9 & 0.9 & 0.8 & 0.7 & 0.6 & 0.5 & 0.3 & 0.3 & 0.2 & 0.2 \\
\hline \multicolumn{13}{|c|}{ Fund obligations based on existing and prospective credit (in millions of SDRs) } \\
\hline Principal & 10.5 & 21.1 & 21.1 & 21.1 & 42.8 & 46.3 & 41.8 & 41.8 & 41.8 & 20.1 & 6.0 & 0.0 \\
\hline Charges and interest & 0.1 & 1.3 & 1.4 & 1.3 & 1.2 & 1.1 & 1.0 & 0.8 & 0.6 & 0.4 & 0.3 & 0.2 \\
\hline \multicolumn{13}{|l|}{ Total obligations based on existing and prospective credit } \\
\hline In millions of SDRs & 10.6 & 22.4 & 22.4 & 22.4 & 44.0 & 47.5 & 42.7 & 42.6 & 42.4 & 20.5 & 6.3 & 0.2 \\
\hline In millions of US\$ & 16.8 & 35.7 & 35.7 & 35.5 & 69.6 & 75.0 & 67.5 & 67.2 & 66.9 & 32.4 & 9.9 & 0.4 \\
\hline In percent of gross international reserves & 0.3 & 0.6 & 0.4 & 0.3 & 0.6 & 0.5 & 0.4 & 0.4 & 0.3 & 0.2 & 0.0 & 0.0 \\
\hline In percent of exports of goods and services & 0.1 & 0.2 & 0.2 & 0.2 & 0.3 & 0.3 & 0.3 & 0.3 & 0.2 & 0.1 & 0.0 & 0.0 \\
\hline In percent of debt service & 4.5 & 6.1 & 6.5 & 5.0 & 7.8 & 7.2 & 3.4 & 4.0 & 3.1 & 1.3 & 0.3 & 0.0 \\
\hline In percent of GDP & 0.0 & 0.1 & 0.1 & 0.1 & 0.1 & 0.1 & 0.1 & 0.1 & 0.1 & 0.0 & 0.0 & 0.0 \\
\hline In percent of quota & 2.9 & 6.1 & 6.1 & 6.1 & 11.9 & 12.9 & 11.6 & 11.5 & 11.5 & 5.6 & 1.7 & 0.1 \\
\hline \multicolumn{13}{|l|}{ Outstanding Fund credit ${ }^{1}$} \\
\hline In millions of SDRs & 363.2 & 461.3 & 440.2 & 419.1 & 376.3 & 330.0 & 264.4 & 186.9 & 109.4 & 53.6 & 11.9 & 0.0 \\
\hline In millions of US\$ & 577.1 & 734.8 & 699.5 & 664.7 & 595.8 & 521.4 & 417.8 & 295.3 & 172.9 & 84.7 & 18.8 & 0.0 \\
\hline In percent of gross international reserves & 10.6 & 11.5 & 8.3 & 6.3 & 4.8 & 3.6 & 2.6 & 1.7 & 0.9 & 0.4 & 0.1 & 0.0 \\
\hline In percent of exports of goods and services & 3.9 & 4.4 & 3.8 & 3.4 & 2.8 & 2.3 & 1.7 & 1.1 & 0.6 & 0.3 & 0.1 & 0.0 \\
\hline In percent of debt service ${ }^{1}$ & 155.8 & 126.0 & 127.6 & 94.2 & 66.9 & 50.4 & 20.8 & 17.7 & 8.0 & 3.3 & 0.6 & 0.0 \\
\hline In percent of GDP & 1.5 & 1.7 & 1.5 & 1.3 & 1.1 & 0.9 & 0.7 & 0.4 & 0.2 & 0.1 & 0.0 & 0.0 \\
\hline In percent of quota & 98.4 & 125.0 & 119.3 & 113.6 & 102.0 & 89.4 & 71.7 & 50.7 & 29.7 & 14.5 & 3.2 & 0.0 \\
\hline Net use of Fund credit (in millions of SDRs) & 108.6 & 96.8 & -22.4 & -22.4 & -44.0 & -47.5 & -42.7 & -42.6 & -42.4 & -20.5 & -6.3 & -0.2 \\
\hline Disbursements & 119.2 & 119.1 & 0.0 & 0.0 & 0.0 & 0.0 & 0.0 & 0.0 & 0.0 & 0.0 & 0.0 & 0.0 \\
\hline Repayments & 10.6 & 22.4 & 22.4 & 22.4 & 44.0 & 47.5 & 42.7 & 42.6 & 42.4 & 20.5 & 6.3 & 0.2 \\
\hline \multicolumn{13}{|l|}{ Memorandum items: } \\
\hline Nominal GDP (in millions of US\$) & 37,879 & 43,396 & 46,994 & 50,922 & 55,384 & 59,359 & 63,506 & 68,014 & 73,106 & 78,699 & 84,882 & 91,598 \\
\hline Exports of goods and services (in millions of US\$) & 14,664 & 16,668 & 18,434 & 19,612 & 21,170 & 22,868 & 24,409 & 26,009 & 27,810 & 29,884 & 32,304 & 34,688 \\
\hline Gross international reserves (in millions of US\$) & 5,432 & 6,390 & 8,449 & 10,602 & 12,457 & 14,370 & 16,201 & 17,825 & 19,458 & 21,047 & 22,961 & 25,090 \\
\hline Debt service (in millions of US\$) & 370.3 & 583.0 & 548.2 & 705.9 & 890.2 & $1,034.7$ & $2,013.2$ & $1,667.2$ & $2,163.2$ & $2,571.4$ & $3,022.5$ & $3,463.9$ \\
\hline Quota (millions of SDRs) & 369 & 369 & 369 & 369 & 369 & 369 & 369 & 369 & 369 & 369 & 369 & 369 \\
\hline
\end{tabular}

s and projections.

${ }^{1}$ Total debt service includes IMF repayments. 
Table 8. Ghana: Proposed Schedule of Disbursements Under the ECF Arrangement, 2009-12 ${ }^{1}$

\begin{tabular}{|c|c|c|}
\hline Amount & Date Available & Condition Necessary for Disbursement \\
\hline SDR 67.65 million & July 15, 2009 & Disbursed on approval of ECF arrangement. \\
\hline SDR 81.50 million & Jun 9, 2010 & $\begin{array}{l}\text { Observance of the performance criteria for } \\
\text { September 30, } 2009 \text { and for December } 31,2009 \text {, and } \\
\text { completion of the combined first and second review } \\
\text { under the arrangement. }\end{array}$ \\
\hline SDR 59.58 million & May 27, 2011 & $\begin{array}{l}\text { Observance of the performance criteria for } \\
\text { June } 30,2010 \text {, and for December } 31,2010 \text {, and } \\
\text { completion of the combined third and fourth review } \\
\text { under the arrangement. }\end{array}$ \\
\hline SDR 59.58 million & $\begin{array}{l}\text { September 15, } \\
2011\end{array}$ & $\begin{array}{l}\text { Observance of the performance criteria for } \\
\text { June } 30,2011 \text {, and completion of the fifth review under } \\
\text { the arrangement. }\end{array}$ \\
\hline SDR 59.58 million & March 15, 2012 & $\begin{array}{l}\text { Observance of the performance criteria for } \\
\text { December } 31,2011 \text {, and completion of the sixth review } \\
\text { under the arrangement. }\end{array}$ \\
\hline SDR 59.56 million & June 15, 2012 & $\begin{array}{l}\text { Observance of the performance criteria for } \\
\text { March } 31,2012 \text {, and completion of the seventh review } \\
\text { under the arrangement. }\end{array}$ \\
\hline SDR 387.45 million & & Total for the ECF arrangement \\
\hline
\end{tabular}

${ }^{1}$ In addition to the generally applicable conditions under the Extended Credit Facility arrangement. 


\title{
APPENDIX I. LETTER OF INTENT
}

November 28, 2011

\author{
Madame Christine Lagarde \\ Managing Director \\ International Monetary Fund \\ Washington, DC 20431
}

Dear Madame Lagarde:

The Ghanaian economy has achieved important macroeconomic stability gains since 2009 . The then sizeable fiscal and external current account deficits have been greatly reduced, inflation has fallen to single digits and the exchange rate has been stabilized. With the start of oil production, and robust performance in the nonoil sector, real GDP is projected to grow by $13 \frac{1}{2}$ percent in 2011 and by 8.5 percent in 2012 . Moreover, a rebasing of the national accounts in 2010 has raised Ghana's national income measures by some 65 percent, moving it to lower middle-income status.

The attached Memorandum of Economic and Financial Policies (MEFP) reports on Ghana's recent economic developments and implementation of Ghana's economic program under the three-year Extended Credit Facility (ECF), which was approved by the IMF Executive Board on July 15, 2009.

All quantitative performance criteria and indicative targets for end-June 2011 were met, with early indications that end-September indicative targets were met as well; only the indicative target for arrears clearance was missed by a very small margin. Based on the strong performance during the first three quarters of the year, the 2011 fiscal deficit could well be below the target of 5.7 percent of (non-oil) GDP. The wage bill, however, is projected to be higher than programmed and is likely to continue to rise in 2012. This mostly reflects the larger-than-expected and unavoidable cost of migration to a new salary structure and retroactive payments to compensate civil servants for delayed migration. Given the projected shortfall in foreign financing, the government is committed to meet these additional obligations while keeping domestic financing in check to contain inflationary pressures. 
Structural reforms have also been progressing, though with some delays. Tax administration was strengthened, resulting in more buoyant revenue mobilization. The government has tackled domestic arrears successfully by implementing a completed arrears management strategy. Public financial management reforms to improve expenditure controls continue more slowly. Some steps to improve financial stability through the supervision of problem banks are on track for delayed completion in 2012. The government is committed to implementing these reforms as soon as possible.

Going forward, the government's macroeconomic policies will focus on preserving macroeconomic stability and making fiscal space for high-priority investments. To achieve these objectives, the government will keep fiscal deficits at levels that can be prudently financed, while accelerating efforts to fill Ghana's large infrastructure gaps, consistent with the Ghana Shared Growth and Development Agenda (GSGDA). Ghana has become a middle-income country and faces the prospect of declining access to concessional financing. This has prompted the government to negotiate a $\$ 3$ billion ( 8 percent of GDP) Master Facility Agreement (MFA) on non-concessional, but competitive, terms with the China Development Bank (CDB) to finance critical infrastructure investments, including a gas pipeline. The gas pipeline project is urgent to avoid the significant risks of damage to existing oils wells and the environment from gas flaring or reinjection.

These and other components of the government's economic stabilization and reform program are described in the MEFP. Based on these policies, the government requests that the IMF's Executive Board grant a modification of the limit on contracting of non-concessional debt consistent with the government's intention to sign the MFA before the end of 2011. The government also requests approval of proposed new structural benchmarks for the period through June 2012 and modifications to December 2011 and March 2012 performance criteria.

In support of its policies, the government requests that the IMF Executive Board complete the fifth review of Ghana's ECF arrangement and approve disbursements as set forth in the attached schedule to the MEFP. The sixth and seventh reviews are expected to be completed by end-April and end-June 2012, respectively.

The government of Ghana will provide such information as the Fund may request in connection with progress in implementing its economic and financial policies. The government believes that the policies set out in this letter are adequate to achieve the objectives of its program, but it will take any further measures that may become appropriate for this purpose. The government will consult with the Fund on the adoption of these measures, and in advance of revisions to the policies contained in the MEFP, in accordance with the Fund's policies on such consultations. We have no objection to the publication of the staff report for the fifth review under the ECF arrangement, this letter of intent, and the 
attached memorandum of economic and financial policies and technical memorandum of understanding.

$/ \mathrm{s} /$

Dr. Kwabena Duffuor

Minister of Finance and

Economic Planning /s/

Mr. K. B. Amissah-Arthur Governor

Bank of Ghana 


\section{APPENDIX I-ATTACHMENT I}

\section{Memorandum of Economic and Financial Policies}

1. This document summarizes the government's assessment of Ghana's current economic situation and performance of the economy through September 2011, discusses the projections and policy stance for the remainder of the year and sets out policy objectives for 2012 and the medium term. These objectives are informed by and consistent with the Ghana Shared Growth and Development Agenda.

\section{RECENT ECONOMIC DEVELOPMENTS AND OUTLOOK}

\section{Ghana has achieved important gains to macroeconomic stability since the} adoption of its ECF-supported program in mid-2009. The then sizeable fiscal and external current account deficits have been greatly reduced, inflation has fallen to single digits from a peak of more than 20 percent in June 2009, the stock of international reserves has risen to close to $\$ 5$ billion from only $\$ 2$ billion in 2008, and the exchange rate has stabilized. With the start of oil production, and robust performance in the non-oil sector, real GDP is projected to grow by $13 \frac{1}{2}$ percent in 2011. Moreover, a rebasing of the national accounts in 2010 has raised Ghana's national income measures by some 65 percent, moving it to lower middle-income status.

\section{A combination of fiscal consolidation and disinflation has reinforced the} favorable macroeconomic setting in 2011. Through a mixture of new revenue and expenditure measures, the government is on track to reduce the fiscal deficit (after arrears clearance) by about $2 \frac{1}{2}$ percentage points of (non-oil) GDP. At the same time, the Bank of Ghana has reduced its policy rate twice this year-last to $12 \frac{1}{2}$ percent in July-as inflation has stabilized at $8 \frac{1}{2}$ percent. Despite impressive export growth from oil, cocoa, and gold, the external current account deficit for 2011 is projected to remain broadly unchanged at $81 / 4$ percent of GDP, reflecting a strong rebound in imports, implying official reserves of $\$ 5.4$ billion, covering above 3 months of projected 2012 imports. The banking sector remains adequately capitalized and liquid, and nonperforming loans, while still high at 16 percent, are declining.

4. The outlook for $\mathbf{2 0 1 2}$ is positive. External demand for Ghana's exports is expected to remain strong, with oil production volumes projected to rise further, while sizeable infrastructure investments and strong private demand will support broad-based economic activity. The overall economy is projected to grow by more than 8 percent, with the inflation rate remaining broadly stable at around $81 / 2-9$ percent. The main risks to this generally favorable outlook arise from possible adverse developments in world commodity prices and foreign investment flows as a result of a prolonged global slowdown. 


\section{Program Performance}

5. Program performance since the completion of the third and fourth review has been strong. All quantitative performance criteria and indicative targets for end-June 2011 were met, and preliminary data suggest a similarly favorable outcome for the end-September indicative targets; only the target for arrears clearance was missed by a very small margin. Structural reforms have also been progressing, though with some delays and deviations from the previously agreed benchmarks.

6. Based on the strong performance during the first three quarters of the year, the 2011 fiscal deficit target of 5.7 percent of (non-oil) GDP is well within reach. A shortfall in official grants and oil revenues - due to lower production volumes and the impact of carryforward losses on corporate tax payments - is likely to be offset by overperformance in domestically-generated revenue, reflecting the fruits of recent reforms. The wage bill, however, is projected to be almost 1 percentage points of GDP higher than programmed, reflecting (i) the larger-than-expected cost of migration to the single spine salary structure including the higher-than-expected base pay increase agreed in 2011; and (ii) a retroactive payment to compensate civil servants for delayed migration (beyond the effective date of January 1,2010). The government is committed to meet these additional obligations without exceeding the agreed deficit target, as a share of GDP.

\section{ECONOMIC ObJectives ANd POLICIES For 2012 AND THE Medium Term}

\section{The government's macroeconomic policy agenda will focus on three complementary objectives:}

- Preserving the gains of macroeconomic stabilization and fiscal consolidation achieved since 2009 .

- Making fiscal space for high-priority investments to spur long-run growth and development.

- Maintaining inflation in single digits.

To achieve these objectives, the government will keep fiscal deficits at levels that can be prudently financed, while accelerating efforts to fill Ghana's large infrastructure gaps, consistent with the Ghana Shared Growth and Development Agenda (GSGDA). This will help set Ghana's economy on a higher trajectory of development and poverty reduction across the country. In light of Ghana's lower middle-income status and prospects of declining access to concessional financing, the government has negotiated, and intends to sign by end2011, a US\$3 billion (8 percent of GDP) Master Facility Agreement (MFA) on nonconcessional terms with the China Development Bank. The funds will be used to finance critical infrastructure investments, including a gas pipeline that is urgently needed to avoid the significant risks to existing oil wells from reinjection. 


\section{A. Fiscal Policy}

8. In the 2012 budget, the key priority will be to continue prudent fiscal policies, while scaling up investment. The government plans to achieve these objectives by:

(i) continuing to mobilize tax revenues through tax administration and policy measures;

(ii) managing the wage bill implications of the single spine migration; and (iii) controlling other recurrent expenditures. The overall deficit will be higher than envisaged at the conclusion of the previous review, but this will reflect higher foreign-financed capital expenditures, as well as the accommodation of larger-than-envisaged costs of the single spine migration. Prudent wage management and various measures on the revenue and expenditure side will ensure that the fiscal stance in 2012 and beyond remains consistent with mediumand long-term debt sustainability.

\section{Strengthening Revenue Mobilization}

9. Tax collections have improved since the launch of the ECF program, but remain low relative to the size of the economy. Non-oil tax revenues as a share of non-oil GDP amounted to only 12 percent in 2009, but could reach $171 / 2$ percent in 2012 . This strengthening in revenue performance stems from a combination of ongoing improvements in tax administration and new tax measures.

\section{Maintaining the Reform Momentum}

10. Appointments of senior officials at the Ghana Revenue Authority (GRA). The Commissioner and Deputy Commissioners are in post.

11. Segmentation of the taxpayer base. The large taxpayers office (LTO) is now fully functional. Companies have been reassigned to the LTO based on turnover and industryspecific criteria (e.g. mining and financial services companies will be registered with the LTO regardless of turnover) to ensure that the LTO covers businesses accounting for at least 70 percent of total tax revenues. Criteria have also been established for identifying medium and small taxpayers, and the registration process will be launched by early 2012 .

12. Integration of VAT and direct taxation under single office heads. Twelve pilot offices in the Greater Accra area tasked with piloting the integration are scheduled to begin taxpayer registration by end 2011 (structural benchmark). Required staff have been identified and fully trained, and the government expects that the pilot system will be fully operational by March 2012 .

13. VAT turnover threshold. The threshold for VAT reporting will be increased in the 2012 budget from $\mathrm{GH} \varnothing 90,000$ to $\mathrm{GH} \notin 120,000$, consistent with IMF technical assistance advice. Smaller taxpayers, who are excluded from the main VAT regime, are currently covered by a temporary regime which requires payment of a 3 percent VAT, in addition to a 
2 percent income tax rate. This arrangement will be replaced by a new regime in the 2012 budget based on a 5 percent tax on turnover.

14. Streamlining of tax exemptions. All domestic VAT zero-rating has been eliminated and replaced by exemptions. The government has completed a review of procedures for granting tax exemptions, in order to reduce the resulting foregone revenues. Exemptions granted through the Ghana Investment Promotion Center have been eliminated, unless approved by Parliament. Power to grant exemptions is also being closely monitored and the government may further limit exemptions through administrative action.

15. Strengthening of customs administration. Collections from taxes on imported goods have outperformed budget targets, with combined import VAT and duties rising from 4.4 percent of non-oil GDP in 2010 to an estimated 5.6 percent in 2011 . New legislation will be proposed to modernize customs administration. Already, the valuation system for goods at the border has been upgraded, and further reductions in the number of bonded warehouses are envisaged as a means to reduce tax avoidance.

16. Revised VAT legislation. The government is seeking accelerated approval of a VAT bill submitted to Parliament in May 2011. The bill includes the extension of VAT to feebased financial services and is expected to be passed in time to be fully implemented in 2012 . This measure is expected to generate an annual revenue yield of $\mathrm{GH} \notin 107$ million (0.2 percent of non-oil GDP).

Moving forward with new policy measures

17. In the 2012 budget, the government will propose a number of new measures to further strengthen revenue performance:

18. Natural resource taxation. Consistent with IMF technical assistance recommendations, the government plans to make extensive changes to the taxation of activities in the natural resource sector, in particular mining.

- Following established practice in the industry - and in the oil and gas sector - the corporate tax rate for mining will be increased from 25 to 35 percent, with a revenue yield estimated at GH $\varnothing 313.6$ million (0.6 percent of non-oil GDP).

- The government will also introduce a tax of 10 percent on windfall profits. The threshold for defining windfall profits still needs to be determined.

- The government will establish a uniform regime for capital allowances including the annual allowance of 20 percent for five years for mining, as is the case in the oil and gas sector. 
- New rules will be established to ensure ring fencing of projects in miningpreventing losses from being transferred among projects. The absence of such rules has allowed companies undertaking a series of projects to deduct costs from new projects against profitable ventures yielding taxable income.

- The government will adopt OECD guidelines for transfer pricing. Companies will be required to disclose related-party transactions and an appropriate penalty for nondisclosure will be applied.

19. Capital gains tax. The government will review the existing law on mergers and acquisition with a view of enhancing its ability to collect capital gains taxes from the transfer of interest between non-resident business entities. Amendments to the existing legislation will be submitted to parliament for its approval before end-2012.

20. Personal income tax. The PAYE tax-free thresholds will be increased and an expansion of income bands will be proposed in the 2012 budget. Inflation can have a substantial effect on the rate structure of a progressive personal income tax, with more taxpayers facing higher marginal rates. The government however, is committed to making these changes revenue neutral. In addition, there will be projected revenue gains of about $\mathrm{GH} \varnothing 115$ million ( 0.2 percent of GDP) from income tax payments on deferred wage payments from the single spine migration (see 926 ).

21. Other tax measures. The government will also implement a tax stamp on the self employed and increase the vehicle inspection tax. On the other hand, a number of measures are planned to reduce the tax burden on key private sector taxpayers. The tax holiday for the Ghana Stock Exchange will be extended by an additional five years, to encourage saving, financial sector development, and the use of equity financing. The government will honor its commitment to eliminate the national fiscal stability levy. These measures together are expected to be broadly revenue neutral.

22. The combined revenue yield from all new tax policy measures is expected to be at least 0.8 percent of GDP. This, however, is a conservative estimate because the potential gains from the windfall profit tax, uniform tax regime on capital allowances, new rules on transfer pricing, and changes in capital gains taxes have not yet been quantified.

\section{Expenditures: Balancing Development Needs with Expenditure Control}

\section{Creating fiscal space for scaling up investment}

23. Meeting GSGDA priorities. The MFA with the China Development Bank will cover a number of important projects consistent with GSGDA priorities. Projects in the oil and gas sector will begin urgently, as the government wants to avoid further damage to the oil reservoir at the Jubilee field. The government will ensure that each project is supported by robust feasibility and financial viability studies as required under the MFA. The feasibility 
studies will be contracted by the relevant ministries and agencies and subjected to a reliable third-party assessment, with the MoFEP overseeing the process. Government will further ensure that the phasing of disbursements for these and other projects is consistent with the agreed program targets and medium-term fiscal plans to preserve debt sustainability. Total disbursements on public and publicly guaranteed nonconcessional loans will not exceed $\$ 1,243$ million in 2012, of which $\$ 750$ million (corresponding to MFA funding) will directly finance the central government budget.

24. Strengthening investment planning and debt management. The government realizes that further scaling up will need to be supported by an integrated investment and financing strategy. To this end:

- A Public Investment Division (PID) has been established in the Ministry of Finance and Economic Planning (MoFEP). The division's role is to: (i) establish and maintain a rolling medium-term public investment plan, consistent with GSGDA priorities; (ii) evaluate, assess, and monitor major investment projects; and (iii) advise on the selection of Public-Private Partnership (PPP) projects. For this purpose, a new PPP policy framework has been developed with support from the World Bank and the UK Department for International Development.

- Debt management capacity and policies are being strengthened. A review of the debt management division was conducted with the objective of identifying skills needs and ensuring effective coordination of the various units. The reorganization of the debt management division is nearing completion. A draft second annual debt management strategy has been formulated and should be ready for publication at end-2011, as foreseen under the program.

- $\quad$ Policy guidelines will be established, by end-April 2012, to strengthen the coordination between PID, the Debt Management Division, and the Economic Research and Forecasting Division at the Ministry of Finance and Economic Planning (structural benchmark). The guidelines will cover coordination among these entities with respect to: (i) debt sustainability and risk analyses, including assessing risks associated with explicit contingent liabilities, such as guarantees on debt contracted by sub-national governments or state-owned entities, or from PPPs; and (ii) establishing multi-year investment programs and borrowing plans that are consistent with the medium term expenditure framework and the public debt management strategy. The government will request technical assistance from the Fund or other development partners to support these efforts.

- $\quad$ The guidelines will also spell out the role and rules governing the recently established joint Public Debt Management Committee (PDMC) in advising the Minister of Finance and Economic Planning on public borrowing decisions. A key role of the PDMC will be to ensure that: (i) all public investment projects are supported by 
robust cost-benefit analyses; and (ii) financing arrangements are consistent with the government's debt management strategy and subjected to a thorough legal assessment by the Ministry's legal office.

\section{Managing the wage bill}

25. Migration to the single spine salary structure. The migration, which began in 2010 to strengthen pay equity and easier salary negotiations, will be completed by end-2011. As of mid-November 2011, 97.4 percent of public servants have been migrated onto the single spine. The cost of the migration proved difficult to estimate, as the mapping of employees from the old to the new pay structure was partially determined by an evaluation of job requirements, but exact placement in the new structure was the result of consultations. Delays in concluding these negotiations, as well as logistical and organizational challenges, resulted in the accumulation of sizeable deferred wage payments. While the exact amount of retroactive payments will only be known once the migration is complete, the current estimate is about GH $\varnothing 1.25$ billion (including some deferred payments related to the 2011 base pay increase).

26. The government is planning to phase payment of retroactive single spine awards over a period of 10 months. A total payment of about $\mathrm{GH} \notin 260$ million $(0.5$ percent of nonoil GDP) will be made in the last quarter of 2011, and the remaining GHф 990 million (1.5 percent of nonoil GDP) will be spread over the first 7 months of 2012. A first increase in pay relativity since the inception of the single spine salary structure will also be implemented in 2012. The cost of the relativity adjustment (estimated at 9.3 percent of the wage bill) and base pay increases will be covered by savings from a payroll audit that will be completed by May 2012 (new structural benchmark), such that the overall wage bill will not exceed GHф 5,050 million (7.8 percent of nonoil GDP).

\section{Inclusion of non-security subvented agencies in the Integrated Personnel and} Payroll Database (IPPD). The government has completed the migration of 103 out of the 132 subvented agencies, covering approximately 21,598 employees. The measure, which in part aims at establishing the overall number of employees of subvented agencies, was delayed by the simultaneous migration to the single spine in the context of limited resources. Once the single spine migration is completed, the government will accelerate IPPD migration and expects completion by June 2012. A review of a partial list of subvented agencies was conducted by independent consultants to determine whether they should remain on government subvention, liquidated or commercialized. The report recommended that 12 out of the 132 subvented agencies should be closed, 71 should remain on government subvention while 16 could be partially or fully commercialized. Recommendations for action were submitted to Cabinet in March 2011, and the government plans to start implementation by June 2012. 


\section{Strengthening Public Financial Management}

28. Arrears clearance and management. Following the renewed rise in domestic payment arrears in 2010, the government has made substantial progress in arrears clearance. After exceeding its target by a large margin in the first half of the year, the September indicative target was missed by a small margin, and the government remains on track to clear all arrears by early 2013. An arrears clearance strategy, involving the combination of cash payments and the issue of special purpose bonds and promissory notes, has been developed (structural benchmark), and a comprehensive database tracking the stocks and flows is being maintained by the Budget Department of the MoFEP, with monthly status reports presented to Cabinet. To prevent the emergence of new arrears, a presidential circular has mandated all line ministries to secure commencement certificates before committing the government to any contractual obligation.

\section{Ghana Integrated Financial Management Information System (GIFMIS).}

GIFMIS implementation is at the heart of the government's PFM agenda, but its pace has stayed behind earlier expectations, related to the complexity of the undertaking and challenges in procurement and system compatibility. Recent progress has been made with the set up of a general ledger and the rollout of a new unified chart of accounts, compliant with GFSM 2001. By the end of October 2011, the general ledger will be rolled out in 14 pilot ministries, departments, and agencies (MDAs) (structural benchmark). All MDAs now enjoy full network connectivity, which will facilitate the roll out of GIFMIS.

30. Improvement of budget processes. The government has adopted monthly expenditure ceilings to facilitate cash management at the MoFEP. After further consideration, it now favors postponing the implementation of quarterly expenditure ceilings for MDAs until spending discipline has been firmly established. The government has also communicated indicative spending ceilings ahead of the 2012 budget preparation process, based on the new unified chart of accounts to support the establishment of realistic spending plans (structural benchmark).

31. Cash management. Large float transactions (discrepancies) of about 825 million (1.5 percent of non-oil GDP) arose in June, pointing to ongoing challenges in cash management. While a treasury main account exists, the government's cash balances are distributed over a large number of accounts (over 4000) at the Bank of Ghana and commercial banks. This has led to situations, as in June, where the treasury main account had insufficient funds to affect payment, creating a discrepancy between the deficit and total financing. To prevent such problems in the future, the government will accelerate the transition to a Treasury Single Account (TSA) which will ensure that all Government funds represented by account balances are consolidated to finance payments. This will minimize situations where some accounts have idle balances whilst others have insufficient balances to finance payments. The following summarizes the operational framework: 
- The TSA will be part of the Real Time Gross Settlement (RTGS) and will be credited directly with revenue received from commercial banks on behalf of the Ghana Revenue Authority (GRA) and others.

- Payments would be made through the Ghana Integrated Financial Management Information System (GIFMIS) directly to beneficiaries through their bankers in the RTGS.

- In the event of an insufficient balance on the TSA to fund payments, BoG will provide funding under pre-arranged terms.

- All transactions will bear their respective expenditure and revenue codes to aid reporting.

- The RTGS will also send electronic statements and confirmation of revenue/expenditure to the GIFMIS to facilitate automated reconciliation.

\section{Tight fiscal monitoring and budget execution will ensure that the 2012 fiscal}

targets are achieved. The better fiscal outcomes in 2011 are partly due to improvements in monitoring fiscal developments, particularly with respect to goods and services and discretionary domestic investment spending, and the implementation of tighter commitment controls. The government will pursue these vigorously also in 2012. At the same time, the government realizes that there are risks to the fiscal outturn, for example, from higher wage payments in the event that the savings from the payroll audit are smaller than anticipated. These risks should be offset by conservative revenue estimates, which do not include gains from a number of measures that have yet to be quantified. Nevertheless, to guard against budget overruns, the government will hold back on spending commitments on goods and services and domestically-financed capital spending, until uncertainties about the outcome of the payroll audit and implementation of new tax measures are resolved.

\section{B. Energy Sector Reforms}

33. Cost-recovery pricing of energy products remains a priority. The government's strategy is to closely monitor energy prices, make adjustments when needed, and smooth price changes to the consumer.

- $\quad$ Electricity sector. Since June 2010, the electricity tariff structure has been reviewed on a quarterly basis. Tariffs were adjusted by 7 percent in September 2011 to costrecovery levels.

- $\quad$ Refined petroleum products. A Petroleum Price Risk Management hedging program was adopted by Cabinet in October 2010 to stabilize domestic pump prices and mitigate the impact of higher world prices on consumers. The government purchases monthly call options that generate revenues when oil prices rise. The proceeds are 
used to cover the cost of subsidizing pump prices, which are reviewed on a bimonthly basis to decide on any needed adjustment. The last price adjustment-a 30 percent increase - occurred in January 2011. While sizeable hedging gains in the first half of the year allowed the subsidies to be covered through July, subsequent subsidies left a gap of about GH $\not 100$ million ( 0.2 percent of GDP) at end-October, which has been settled from the budget. To avoid costly subsidies, the government will increase petroleum prices to full cost-recovery levels. Thereafter, the government will adjust prices on a monthly basis, if needed to avoid subsidies, irrespective of profits (or losses) from its hedging transactions, which will be included in the budget to ensure transparency.

\section{Monetary, Financial and Exchange Rate Issues}

34. The Bank of Ghana's inflation targeting regime continues to successfully anchor inflation expectations. The BoG's inflation forecast suggests that the end-year target of 9.0 percent will be achieved, notwithstanding the recent depreciation of the Ghana cedi. Looking forward, the BoG aims at keeping inflation broadly stable, with the center point of the target band modestly reduced to 8.7 percent in December 2012. It will stand ready to adjust its policy rate in support of this target should upside risks to inflation become pronounced.

35. The BoG stands ready to adjust policies in support of its inflation target. Thus far, the BoG has taken advantage of balance of payments surpluses to strengthen the reserve cover to above 3 months of imports and has accommodated an expansion of private sector credit by about 12 percent, in real terms. However, the cost of sterilizing the liquidity impact has been significant and discussions are ongoing on a proposal to share the cost of sterilization with the budget. More recently, lower than expected oil revenues and an unwinding of domestic bond holdings by foreign investors has led the BoG to intervene in the opposite direction to contain undue exchange rate volatility, implying a lower level of reserve accumulation than earlier anticipated. Going forward, the BoG aims to develop a program for foreign exchange market monitoring and intervention policies to help manage liquidity and intends to request IMF technical assistance in these areas.

36. In the financial sector the priority continues to strengthen banks' risk management capacity. Several specific actions are underway:

- Tema Oil Refinery (TOR) debt to Ghana Commercial Bank (GCB). The cedi component of the debt has been cleared and the foreign-exchange components have been reduced to about $\$ 50$ million. In July 2011, GCB hired two internationally reputed consultants which are providing advice on a general reassessment of risk management practices in the bank. The full implementation of a new risk management approach is expected to be rolled out in all branches by end-2012. 
- Audit of banks. The BoG remains fully committed to resolving the issues of remaining problem banks. The independent audit of these banks will have to be delayed, however, as will the development of new banking supervisory guidelines, because the selection of the external consultant to oversee the process took longer than expected. Provided the long-term consultant in banking supervision assumes duty at the Banking Supervision Department in January 2012, as planned, international firms to conduct the independent audit will be appointed by end-March 2012 (new structural benchmark), with the plan to complete the audit by the end of the year. All banks have met the minimum capital requirement of GHф25.0 million and are expected to comply with the GHф 60 million requirement by end 2012 .

- Amendments to the financial sector legal framework. The BoG has contracted legal consultants to draw up regulations to provide for the effective implementation of financial sector laws by addressing enforcement and emerging issues of market/industry development, including central bank resolution powers. The priority focus will be on the Banking Act (Act 673) and Foreign Exchange Act (Act 723), followed by a consolidation of the Banking Act (Act 673) and the Banking (Amendment) Act (Act 738), which should provide an opportunity to address regulatory gaps. The Bank expects a report on the draft regulations to be submitted in the first quarter of 2012, to be followed by the submission of the Legislative Instrument to Parliament for passage.

- BoG equity shareholding in banks. To address the potential conflict of interest as supervisor and part-owner of banks, the Bank of Ghana's shareholding in four banks were transferred to the Financial Investment Trust (FIT) as legal owner of the shares with a mandate to sell the shares. The shares of two banks have since been sold and a third is currently on offer. The fourth bank, which was set up as a statutory corporation, is being converted into a limited liability company, as a necessary first step to list it on the Ghana Stock Exchange.

- Social Security and National Investment Trust (SSNIT) equity shareholding in banks. The Board of SSNIT has agreed to diluting its shareholdings in the banking industry. In this regard, discussions are underway with prospective buyers of its shares in two banks in which it has majority ownership (more than 50 percent) and a merger of a third one with another bank in which it has a minority shareholding.

- The BoG is committed to address remaining deficiencies in AML/CFT. To this end, an AML office was established within the Banking Supervision Department in February 2011, and resources are being recruited to make it fully operational. The office has been working with the FIC and other stakeholders to develop a guideline for the banking industry on AML/CFT measures. The office is receiving technical assistance from the Fund to strengthen the overall legislative and regulatory AML/CFT regime. 
- Strengthening consolidated supervision. A Forum of domestic regulatory authorities, comprising the Bank of Ghana, the National Insurance Commission, the Securities and Exchange Commission and the National Pensions Regulatory Authority has been formed to collaborate and share information to close any regulatory gaps in supervision and reduce systemic risks in the financial sector.

\section{Program Design and Monitoring}

37. Program targets and benchmarks. Quantitative program targets are documented in Appendix Table 1. Structural benchmarks are documented in Appendix Table 2. For the purpose of the program, the exchange rate of the Ghanaian cedi $(\mathrm{GH} \phi)$ to the U.S. dollar will be GH $\notin 1.5374$ (see the attachment II Technical Memorandum of Understanding). 
Appendix MEFP Table 1. Ghana: Quantitative Program Targets (December 2010 - June 2012) '

Cumulative from the beginning of calendar year, unless otherwise indicated)

\begin{tabular}{|c|c|c|c|c|c|c|c|c|c|c|c|c|c|c|c|c|c|}
\hline & & \multirow{3}{*}{$\begin{array}{r}\frac{2010}{\text { Dec. }} \\
\text { Act. }\end{array}$} & \multirow{3}{*}{$\begin{array}{l}2011 \\
\text { Mar. } \\
\text { Act. }\end{array}$} & \multicolumn{4}{|c|}{ Jun. 2011} & \multicolumn{4}{|c|}{ Sep. 2011} & \multicolumn{2}{|c|}{ Dec. 2011} & \multicolumn{2}{|c|}{ Mar. 2012} & \multicolumn{2}{|c|}{ Jun. 2012} \\
\hline & & & & \multicolumn{2}{|c|}{ Prog. ${ }^{2}$ Adjusted } & \multirow[t]{2}{*}{ Act. } & \multirow{2}{*}{\multicolumn{2}{|c|}{$\begin{array}{l}\text { Target Prog }{ }^{3} \\
\text { Met? } 3 / 4 \text { ECF Rev. }\end{array}$}} & Adjusted & Act. & 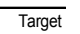 & \multirow{2}{*}{$\begin{array}{l}\text { Prog. }{ }^{2} \\
3 / 4 \mathrm{ECF} \text { Rev. }\end{array}$} & \multirow{2}{*}{$\begin{array}{l}\text { Rev. Prog. }{ }^{2} \\
5 \mathrm{ECF} \text { Rev. }\end{array}$} & \multirow{2}{*}{$\begin{array}{l}\text { Prog. }{ }^{2} \\
3 / 4 \text { ECF Rev. }\end{array}$} & \multirow{2}{*}{$\begin{array}{l}\text { Rev. Prog. }^{2} \\
5 \text { ECF Rev. }\end{array}$} & \multirow{2}{*}{$\begin{array}{l}\text { Prog. }{ }^{3} \\
3 / 4 \text { ECF Rev. }\end{array}$} & Rev. Prog. ${ }^{3}$ \\
\hline & & & & & Target & & & & Target & & Met? 3 & & & & & & 5 ECF Rev. \\
\hline \multicolumn{18}{|c|}{ Quantitative performance criteria } \\
\hline & Overall fiscal deficit of the government (ceiling; millions of cedis) & 3,000 & 560 & 1800 & 1,476 & 528 & Met & 2,420 & 1,735 & 1,132 & Met & 2,919 & 2,741 & 326 & 857 & 733 & 2,273 \\
\hline & Increase in net international reserves of the Bank of Ghana (floor; millions of U.S. dollars) & 937 & -154 & -370 & -340 & -9 & Met & -364 & -364 & -288 & Met & 788 & 518 & -105 & -115 & -275 & -191 \\
\hline & Net change in the stock of domestic arrears (celing, millions of cedis) & 654 & -420 & -693 & -693 & $-1,068$ & Met & $-1,070$ & $-1,070$ & $-1,068$ & Not Met & $-1,167$ & $-1,412$ & -288 & -63 & -403 & -87 \\
\hline \multirow[t]{5}{*}{ ॥ } & Continuous performance criteria & & & & & & & & & & & & & & & & \\
\hline & Non-accumulation of external arrears (ceiling; millions of U.S. dollars) & 0 & 0 & 0 & 0 & 0 & Met & 0 & 0 & 0 & Met & 0 & 0 & & 0 & 0 & 0 \\
\hline & $\begin{array}{l}\text { Contracting or guaranteeing of new medium-to-long-term nonconcessional external debt (ceiling; US\$ } \\
\text { millionst" }\end{array}$ & 196.6 & 0 & 800 & 800 & 278 & Met & 800 & 800 & 278 & Met & 800 & 3400 & 500 & 810 & 500 & 810 \\
\hline & Oil and gas sector ${ }^{5}$ & 0 & 0 & 300 & 300 & 0 & Met & 300 & 300 & 0 & Met & 300 & 1250 & (a) & 0 & 0 & 0 \\
\hline & Other sectors & 196.6 & 0 & 500 & 500 & 278 & Met & 500 & 500 & 278 & Met & 500 & 2150 & 500 & 810 & 500 & 810 \\
\hline \multicolumn{18}{|c|}{ III Inflation consultation } \\
\hline & \multicolumn{17}{|l|}{ Twelve-month consumer price inflation (percent) $^{6}$} \\
\hline & Outer band (upper limit) & & & 12.0 & 12.0 & & & 12.0 & 12.0 & & & 12.0 & 12.0 & 11.7 & 11.7 & 11.7 & 11.7 \\
\hline & Inner band (upper limit) & & & 11.0 & 11.0 & & & 11.0 & 11.0 & & & 11.0 & 11.0 & 10.7 & 10.7 & 10.7 & 10.7 \\
\hline & Central target rate of inflation & 8.6 & 9.1 & 9.0 & 9.0 & 8.6 & Met & 9.0 & 9.0 & 8.4 & Met & 9.0 & 9.0 & 8.7 & 8.7 & 8.7 & 8.7 \\
\hline & Inner band (lower limit) & & & 7.0 & 7.0 & & & 7.0 & 7.0 & & & 7.0 & 7.0 & 6.7 & 6.7 & 6.7 & 6.7 \\
\hline & Outer band (lower limit) & & & 6.0 & 6.0 & & & 6.0 & 6.0 & & & 6.0 & 6.0 & 5.7 & 5.7 & 5.7 & 5.7 \\
\hline \multicolumn{18}{|c|}{ IV Indicative targets } \\
\hline & Net domestic financing of the government (ceiling, millions of cedis) & 2,143 & 580 & 960 & 977 & 600 & Met & 1,550 & 1,609 & 1,215 & Met & 1,545 & 2,361 & 470 & 844 & 130 & 1642 \\
\hline & Net domestic assets of the Bank of Ghana (ceiling; millions of cedis) & -924 & 10 & 503 & 503 & 503 & Met & 643 & 643 & 44 & Met & -279 & 583 & -77 & 635 & -154 & 955 \\
\hline & Poverty-reducing budget expenditures (flloor; millions of cedis) & 2,411 & & 1,100 & 1,100 & 1269 & Met & 1,920 & 1,920 & 2,275 & Met & 2,700 & 2,888 & 660 & 858 & 1330 & 1717 \\
\hline
\end{tabular}

'All variables and adjustors to the targets are defined in the Technical Memorandum of Understanding (TMU).

${ }^{2}$ Performance criterion.

Halcalle target except for the inflation consultation mechanism. For June 2012, indicative targets for every indicator.

${ }^{4}$ Measured on an annual basis.

as exploration and production projects in Ghana and to acquire equity stakes in companies

cee is measured on a continuous basis. The outer and inner bands shown for the last month of each quarter apply throughout the respective quarter. 
Appendix Table 2. Ghana: Prior Action and Structural Benchmarks Under the ECF Arrangement, 2011-12

(Shaded benchmarks are covered by the $5^{\text {th }}$ review)

\begin{tabular}{|c|c|c|c|}
\hline Measures & Timing & Implementation Status & Macroeconomic Rationale \\
\hline \multicolumn{4}{|l|}{ Prior Action } \\
\hline -Increase domestic petroleum prices & & $\begin{array}{l}\text { Prior action for the completion of the fifth review } \\
\text { (see MEFP }\{33 \text { ) }\end{array}$ & $\begin{array}{l}\text { To avoid energy sector SOE losses, } \\
\text { that have historically posed serious } \\
\text { burdens on the budget. }\end{array}$ \\
\hline \multicolumn{4}{|l|}{$\underline{\text { Structural Benchmarks }}$} \\
\hline \multicolumn{4}{|l|}{ 1. Tax policy and revenue administration } \\
\hline $\begin{array}{l}\text { - Complete comprehensive reviews of } \\
\text { zero-rated VAT items and the nature and } \\
\text { scope of tax exemptions and } \\
\text { discretionary waivers. }\end{array}$ & $\begin{array}{l}\text { End-Sep. } \\
2009\end{array}$ & Implemented. & $\begin{array}{l}\text { Strengthen revenue mobilization as } \\
\text { part of the fiscal consolidation } \\
\text { strategy. }\end{array}$ \\
\hline $\begin{array}{l}\text { - Appoint senior Ghana Revenue } \\
\text { Authoritiy (GRA) management } \\
\text { (commissioners and deputy } \\
\text { commissioners) and adopt criteria to } \\
\text { ensure that the Large Taxpayer Unit } \\
\text { (LTU) covers businesses accounting for } \\
\text { at least } 70 \text { percent of total tax revenues. }\end{array}$ & $\begin{array}{l}\text { End-Jun. } \\
2010\end{array}$ & Implemented. & As above. \\
\hline $\begin{array}{l}\text { - Adopt a tax regime for small business } \\
\text { taxpayers for introduction in the } 2012 \\
\text { fiscal year. }\end{array}$ & $\begin{array}{l}\text { End-Jun } \\
2011\end{array}$ & $\begin{array}{l}\text { Implemented. Criteria for identifying medium and } \\
\text { small taxpayers have been identified, and the } \\
\text { registration process will be launched by early } 2012 \\
\text { (See MEFP, } \uparrow 11 \text { ) }\end{array}$ & As above. \\
\hline
\end{tabular}

\footnotetext{
${ }^{1}$ Includes earlier benchmarks not fully implemented by the time of the third and fourth review.
} 
- Pioneer the integration of VAT and income tax in 11 pilot offices in Greater Accra. Staff will be in common offices, under a single head of office, and with a common audit process.

- Extend self-assessment for tax purposes to all medium and large taxpayers.
End-Dec Benchmark (for sixth review).

$2011 \quad$ (See MEFP, ๆ12)
As above.

End-June $\quad$ Benchmark (for seventh review) $\quad$ As above.

\section{Public expenditure management}

- Roll out GIFMIS to 14 selected pilot

MDAs.
Reintroduce quarterly expenditure ceilings for planning purposes, while retaining the current monthly cash ceiling.

- Design and implement a revised 2012

MTEF/budget calendar to allow for a

Cabinet discussion in August/September

2011 to frame 2012 budget priorities

(across GSGDA goals) and provision of indicative resource ceilings to ministries, departments, and agencies (MDAs) in July 2011.
End-March

2011
Delayed. Reset Benchmark (for sixth review) Roll out to pilots delayed; will be piloted within the CAGD and 14 MDAs by October 2011 and rolled out to all ministries starting January 2012.

(See MEFP, $\llbracket 29$ )

\section{End-October Not implemented. Implementation of quarterly 2011 expenditure ceilings for MDAs will be postponed until spending discipline has been firmly established. (See}

Strengthen monitoring and control of budget execution.

As above.

MEFP, $\mid 30)$

Benchmark (for sixth review).

As above.

December

2011 


\begin{tabular}{|c|c|c|c|}
\hline \multicolumn{4}{|l|}{ 4. Arrears management } \\
\hline $\begin{array}{l}\text { - Assign organizational responsibility for } \\
\text { maintaining a comprehensive central } \\
\text { database of central government arrears } \\
\text { and public liabilities in regard of SOE } \\
\text { obligations. The responsible unit will } \\
\text { compile monthly reports, tracking } \\
\text { outstanding liabilities, new claims, and } \\
\text { settlements. Quarterly reports on claims } \\
\text { to be presented to Cabinet, with a first } \\
\text { report by end-June } 2011 \text {. }\end{array}$ & $\begin{array}{l}\text { End-June } \\
2011\end{array}$ & $\begin{array}{l}\text { Implemented } \\
(\text { See MEFP, } \mid 28)\end{array}$ & $\begin{array}{l}\text { To restore credibility to the budget } \\
\text { process and address the liquidity } \\
\text { problems of contractors, banks, } \\
\text { and SOEs associated with domestic } \\
\text { arrears and other unpaid fiscal } \\
\text { obligations. }\end{array}$ \\
\hline $\begin{array}{l}\text { Develop a strategy for regularizing } \\
\text { arrears and other liabilities within the } \\
\text { medium-term macro-fiscal framework. }\end{array}$ & $\begin{array}{l}\text { End-Sept. } \\
2011\end{array}$ & $\begin{array}{l}\text { Implemented. } \\
\text { (See MEFP, }\{28)\end{array}$ & As above \\
\hline \multicolumn{4}{|l|}{ 5. Public debt management } \\
\hline $\begin{array}{l}\text { - Publish a second annual debt } \\
\text { management strategy. }\end{array}$ & $\begin{array}{l}\text { End-Dec. } \\
2011 .\end{array}$ & $\begin{array}{l}\text { Benchmark (for sixth review). } \\
\text { (See MEFP, } ₫ 24 \text { ) }\end{array}$ & As above \\
\hline $\begin{array}{l}\text { - Establish policy guidelines to } \\
\text { strengthen the coordination between the } \\
\text { Public Investment Division, the Debt } \\
\text { Management Division, and the } \\
\text { Economic Research and Forecasting } \\
\text { Division at the Ministry of Finance } \\
\text { and Economic Planning. }\end{array}$ & $\begin{array}{l}\text { End-April } \\
2012 .\end{array}$ & $\begin{array}{l}\text { New benchmark (for sixth review). } \\
\text { (See MEFP, } \mid 24 \text { ) }\end{array}$ & As above \\
\hline \multicolumn{4}{|c|}{ 6. Public sector reform and payroll management } \\
\hline $\begin{array}{l}\text { - Migrate to the automated payroll } \\
\text { system (IPPD2/3) all remaining non- } \\
\text { security subvented agencies. }\end{array}$ & $\begin{array}{l}\text { End-July } \\
2010\end{array}$ & $\begin{array}{l}\text { Delayed. To be completed by end-June } 2012 . \\
\text { (See MEFP, } \llbracket 27 \text { ) }\end{array}$ & $\begin{array}{l}\text { To strengthen oversight and } \\
\text { control of the high and growing } \\
\text { public payroll. }\end{array}$ \\
\hline $\begin{array}{l}\text { - Complete pay comparability survey } \\
\text { for public and private sectors for a } \\
\text { sizeable number of public sector career } \\
\text { streams. }\end{array}$ & $\begin{array}{l}\text { End-June } \\
2011\end{array}$ & Implemented & As above. \\
\hline
\end{tabular}




$\begin{array}{lll}\text { - Complete civil service payroll audit to } & \begin{array}{l}\text { End-May } \\ 2012\end{array} & \text { New benchmark (for seventh review). } \\ \text { remove ghost workers. } & \text { To contain budgeted wage costs. }\end{array}$

\section{Energy sector management}

- Management of petroleum product

End-Sept.

2011
Delayed. Reset Benchmark (for sixth review). Prices will be adjusted to full cost recovery by end-January 2012 and maintained thereafter through monthly price adjustments, if needed to avoid subsidies.

\section{Monetary and financial policy}

- Develop strategy to fully strengthe

Ghana Commercial Bank balance sheet.

End-July

2010

- Conduct an independent audit of remaining problem banks.

- Appoint international firms to conduct independent audit of remaining problem banks.

- Develop a strategy for addressing problem banks.

- Submit for parliamentary approval amendments to the Banking Laws to close 2012 regulatory gaps, including with respect to bank resolution options.

End-Dec.

2011
To avoid energy sector SOE losses, that have historically posed serious burdens on the budget. (See MEFP, 『33)

End-Nov. Delayed. The external consultant has been appointed As above.

$2011 \quad$ with delay and will start the audit in January 2012.

Expected completion of the audits by December 2012

(See MEFP, \36)

End-Mar. New benchmark (for sixth review)

As above

\section{$2012 \quad$ (See MEFP, q36)}

Reset Benchmark to March 2012 (for sixth review).

As above.

The external consultant has been appointed with delay

and will assist in formulating the strategy.

(See MEFP, $\llbracket 36$ )

End-Mar. Benchmark (for sixth review)

As above.

(See MEFP, q36) 


\section{APPENDIX I-ATTACHMENT II}

\section{TECHNICAL MEMORANDUM OF UNDERSTANDING FOR THE 2011-12 EXTENDEd CREDIt FACILITY (ECF) ARRANGEMENT}

1. This technical memorandum of understanding (TMU) defines the variables subject to quantitative targets (performance criteria and indicative targets), as specified in the authorities' Letter of Intent (LOI) of November 28, 2011. It also describes the methods to be used to assess the program performance and the information requirements to ensure adequate monitoring of the targets. The authorities will consult with the Fund before modifying measures contained in this letter, or adopting new measures that would deviate from the goals of the program, and provide the Fund with the necessary information for program monitoring.

2. Program exchange rate: The exchange rates for the purpose of the program of the Ghanaian cedi $(\mathrm{GH} \phi)$ to the U.S. dollar will be GH $\phi 1.5374$ per US\$1, which is calculated as the average of buying and selling exchange rates reported by banks to the Bank of Ghana. The exchange rates to other currencies will be calculated as the average of buying and selling exchange rates against the U.S. dollar.

\section{Quantitative Program Indicators}

3. For program monitoring purposes, the performance criteria are set for end-June 2011, end-December 2011, and end-March 2012, while indicative targets are set for end-March 2011, end-September 2011, and end-June 2012. Performance criteria, indicative targets, and adjusters are calculated as cumulative flows from the beginning of the calendar year.

4. The performance criteria under the arrangement are:

- a ceiling on the overall fiscal deficit of the government, measured in terms of financing;

- a floor on the net international reserves of the Bank of Ghana;

- a ceiling on the net change in the stock of domestic arrears;

- a continuous zero ceiling for the accumulation of new external arrears; and

- a ceiling on the contracting or guaranteeing of new external nonconcessional debt

5. Indicative targets are established as:

- a ceiling on the net domestic financing of the government;

- a floor on non-oil tax revenue collection

- a ceiling on the net domestic assets of the Bank of Ghana; and

- a floor on poverty-reducing government expenditures. 
6. A target is set for the twelve-month rate of consumer price inflation, with triggers on discussions or consultations with the Fund if inflation moves outside specified inner and outer bands.

\section{A. Government}

7. Definition: The government is defined as comprising the central government, all special funds (including the Education Trust Fund, the Road Fund, the District Assemblies Common Fund, and the National Health Insurance Fund), and all subvented and other government agencies that are classified as government in the Bank of Ghana (BoG) Statement of Accounts (SOA). The Social Security and National Insurance Trust (SSNIT) and public enterprises, including Cocobod, are excluded from the definition of government

8. The government's total tax revenue includes all revenue collected by the Ghana Revenue Authority (GRA) (direct taxes, indirect taxes, trade taxes) whether they result from past, current, or future obligations. Receipts are recorded on a cash basis.

9. Oil tax revenue is defined as the government's net proceeds from the sale of oil, including corporate tax and royalties paid by oil companies, excluding any revenue associated with GNPC's carried interests in oil fields.

10. Non-oil tax revenue will be measured as total government tax revenue less oil tax revenue (as defined in paragraph 9).

11. The fiscal deficit is measured as total financing extended to the government (as defined in paragraph 7 above), comprising the sum of net foreign borrowing (as defined in paragraph 15 below), net domestic financing (defined in paragraph 14 below), exceptional financing (including HIPC and MDRI relief against loan repayments falling due), and receipts from net divestitures.

12. Domestic payments arrears will be measured as the sum of three components. The first component, arrears to the government's statutory funds, represents any delay of more than one month in revenue transfers to these statutory fund, relative to the normal payment schedule (typically monthly or quarterly, and defined as a specific percentage of the previous month or quarter's revenue collections). ${ }^{1}$ The second component, arrears to contractors, is defined as payments in local and foreign currencies that are due and not settled within 30 days after the end of the fiscal year. The third component, wages and pensions arrears, is defined as payments outstanding after the agreed date for payment to staff or the social security fund. In the case of pay awards, arrears are calculated as the amount outstanding at

\footnotetext{
${ }^{1}$ Transfers to the statutory funds are scheduled as follows: (i) District Assemblies Common Fund-quarterly, with a one-quarter lag; (ii) Social Security Fund, National Health Fund, Ghana Education Trust Fund, Road Fund, Petroleum-related Fund,- - monthly, with a one-month lag.
} 
the date at which the award specifies the first payments should be made. Net changes in the stock of arrears to contractors at end-March 2011 are as defined in the TMU attached to the Letter of Intent of May 13, 2010. Starting at end-June 2011, the net change in the stock of arrears to contractors will be measured at each end-quarter as the accumulation of arrears within the current fiscal year, less amounts settled in the current fiscal year in respect of the claims accumulated in previous fiscal years.

13. The government will continue to report poverty-related expenditures. Budgeted poverty spending for these categories will be taken from each year's final appropriations bill and will include only spending financed by the government or from internally generated funds. Actual poverty-related spending will be identified using the last 3 digits of the 15-digit chart of accounts of the CAGD's current NETS. This data will be supplemented with that proportion of transfers to the District Assembly Common Fund, the Ghana Educational Trust Fund, and the Road Fund, which the government considers as poverty-related. Accordingly, actual poverty spending will exclude all donor-supported expenditure.

14. Net domestic financing of government is defined as the change in net credit to government by the banking system (i.e., the Bank of Ghana plus deposit money banks) plus the net change in holdings of treasury bills and other government securities by the nonbank sector, excluding divestiture receipts. Such credit will also exclude treasury bills issued for open market operation purposes from January 1, 2003 onward (the holdings of which are excluded from the BoG Treasury Department's Debt Registry of central government securities, and the proceeds of which are sterilized in deposits held as other BoG liabilities, as defined in the monetary template provided to the IMF on December 3, 2003).

15. Net foreign financing of government is defined as the sum of project and program loans by official donors, commercial external borrowing, minus amortization due.

16. Outstanding net credit to the government by the Bank of Ghana comprises the sum of claims on government (SOA codes 0401 and 050101-4), including overdrafts of the government with the BoG, less government deposits (1101 including the main HIPC Initiative receiving account, and 1202) as defined in the monetary template.

17. Outstanding net credit by deposit money banks comprises deposit money bank (DMB) holdings of government securities at cost of purchase value, as reported by the BoG Treasury Department's Debt Registry, plus overdrafts less government deposits as reported by DMBs in the revised BSD2 report forms (and defined in the Monetary Template), plus deferred accrued interest on their holdings of inflation-indexed bonds.

18. Nonbank financing is the difference between total net cash receipts to the treasury main cash account (issues/redemptions account when it becomes operational) from the sale/repurchase of government securities, less the corresponding net cash value received from the BoG and DMBs as indicated on the Debt Registry by holder at discount value, plus deferred accrued interest on their holdings of inflation-indexed bonds. For each test date, any 
adjustment by the BoG to the data reported by individual DMBs, on account of their misclassification of government or for other reasons, will be reported to the Fund.

\section{B. Consultation Mechanism on Inflation}

19. A consultation mechanism adopted for the twelve-month rate of inflation. Inflation is measured by the headline consumer price index (CPI) published by the Ghana Statistical Services. Quarterly consultation bands are specified in Appendix Tables 1 and 2 attached to the memorandum of economic and financial policies. The bands are defined for each quarter and apply to the three month inflation outturns in each quarter. Appendix Tables 1 and 2 attached to the memorandum of economic and financial policies show the relevant bands for each quarter. Whenever the twelve-month rate of CPI inflation moves outside a specific band, this would trigger a consultation/discussion with the Fund.

20. Breach of the outer band. The authorities will complete consultations with the Executive Board of the Fund on the proposed policy response before requesting further disbursements under the program when the observed twelve-month rate of CPI inflation moves outside the outer band as specified for each quarter in Appendix Tables 1 and 2 of the memorandum of economic and financial policies. The authorities will not be able to request any further disbursements under the ECF arrangement if the observed twelve-month rate of

CPI inflation moves outside of the outer band until the consultation with the Executive Board has taken place. In line with our accountability principles, we are committed to report to the public the reasons for any breach of the outer bands, and our policy response.

21. Breach of the inner band. The authorities will conduct discussions with the Fund staff when the observed twelve-month rate of CPI inflation falls outside the inner band as specified for each quarter in Appendix Tables 1 and 2 of the memorandum of economic and financial policies.

\section{Bank of Ghana}

22. Net foreign assets are defined in the monetary survey as short- and long-term foreign assets minus liabilities of the BoG that are contracted with nonresidents. Short-term foreign assets include: monetary gold (valued at the spot market rate for gold, US\$/fine ounce, London), holdings of SDRs, reserve position and HIPC Initiative trust investment in the IMF, the HIPC Initiative Umbrella SDR account (all as reported by the IMF), foreign notes and travelers checks, foreign securities, positive balances with correspondent banks, and other positive short-term or time deposits. Short-term foreign liabilities include foreign currency liabilities contracted by the BoG at original maturities of one year or less (including overdrafts), outstanding liabilities to the IMF, and deposits of international institutions at the BoG. Long-term foreign assets and liabilities are comprised of: other foreign assets (BoG statement of accounts code 303), investments abroad (a subset of 60201), other long-term liabilities to nonresidents (a subset of 1103), and bilateral payment agreements (305). All 
values not in U.S. dollars are to be converted to U.S. dollars at the program exchange rate defined in paragraph 2. A more detailed listing of accounts to be included in the measure of net foreign assets is contained in the monetary template referred to in paragraph 14 above.

23. Net international reserves of the $\mathrm{BoG}$ are defined for program monitoring purposes and in the balance of payments as short-term foreign assets of the BoG, minus short-term external liabilities. To the extent that short-term foreign assets are not fully convertible external assets readily available to and controlled by the BoG (that is, they are pledged or otherwise encumbered external assets, including, but not limited to, the HIPC umbrella SDR account), they will be excluded from the definition of net international reserves. Net international reserves are also defined to include net swap transactions (receivable less payable) and exclude all positive foreign currency deposits at the BoG held by resident deposit money banks, public institutions, nonfinancial public enterprises, other financial institutions, and the private sector. All values not in U.S. dollars are to be converted to U.S. dollars at the program exchange rate defined in paragraph 2 .

24. Net domestic assets of the Bank of Ghana are defined as the difference between reserve money and net foreign assets of the BoG, excluding the HIPC Umbrella SDR account, converted from U.S. dollars to cedis at the program exchange rate.

\section{External Debt and Debt Service}

25. For the purposes of this technical memorandum of understanding, the definition of debt is set out in point 9 of the Guidelines on Performance Criteria with Respect to External Debt (Executive Board's Decision No.6230-(79/140), as amended). It not only refers to debt as defined in Point 9 of the Executive Board decision, but also to commitments contracted or guaranteed for which value has not been received. The definition of debt is as follows:

9 (a) For the purpose of these guidelines, the term "debt" will be understood to mean a current, i.e., not contingent, liability, created under a contractual arrangement through the provision of value in the form of financial and nonfinancial assets (including currency) or services, and which requires the obligor to make one or more payments in the form of assets (including currency) or services, at some future point(s) in time; these payments will discharge the principal and/or interest liabilities incurred under the contract. Debts can take a number of forms, the primary ones being as follows:

(i) loans, i.e., advances of money to the obligor by the lender made on the basis of an undertaking that the obligor will repay the funds in the future (including deposits, bonds, debentures, commercial loans and buyers' credits) and temporary exchanges of assets that are equivalent to fully collateralized loans under which the obligor is required to repay the funds, and usually pay 
interest, by repurchasing the collateral from the buyer in the future (such as repurchase agreements and official swap arrangements);

(ii) suppliers' credits, i.e., contracts where the supplier permits the obligor to defer payments until some time after the date on which the goods are delivered or services are provided; and

(iii) leases, i.e., arrangements under which property is provided which the lessee has the right to use for one or more specified period(s) of time that are usually shorter than the total expected service life of the property, while the lessor retains the title to the property. For the purpose of the guideline, the debt is the present value (at the inception of the lease) of all lease payments expected to be made during the period of the agreement excluding those payments that cover the operation, repair, or maintenance of the property.

(b) Under the definition of debt set out in point 9(a) above, arrears, penalties, and judicially awarded damages arising from the failure to make payment under a contractual obligation that constitutes debt are debt. Failure to make payment on an obligation that is not considered debt under this definition (e.g., payment on delivery) will not give rise to debt.

\section{For the purposes of the ceiling on the contracting or guaranteeing of new non-} concessional external debt, external debt is any debt as defined in paragraph 27 , which is denominated in foreign currency, i.e., currency other than Ghanaian cedis $(\mathrm{GH} \phi)$. Similarly, external borrowing is borrowing denominated in foreign currency. ${ }^{2}$

27. Nonconcessional medium- and long-term external debt is defined as external debt contracted or guaranteed by the government (defined in paragraph 7), the BoG, and specific public enterprises (defined in paragraph 31) on nonconcessional terms (see paragraph 34) and denominated in foreign currencies, with an original maturity of more than one year, provided that debt maturing within one year which has been extended beyond one year from its original date, pursuant to the contract which allows for maturity extension, would be considered medium to long term. Medium- and long-term external debt and its concessionality will be reported by the Aid and Debt Management Division of the Ministry

\footnotetext{
2 (A) The term "debt" has the meaning set forth in point No. 9 of the Guidelines on Performance Criteria with Respect to Foreign Debt (Decision No. 6230-(79/140), as amended). This includes overdrafts on accounts with correspondent banks. (B) Excluded from this performance criterion are normal import-related credits, pre-export financing credits of public enterprises, cocoa loans collateralized by cocoa contracts, and individual leases with a value of less than US\$100,000.
} 
of Finance and Economic Planning, and will be measured in U.S. dollars at current exchange rates.

28. For the purpose of the ceiling on the accumulation of external payment arrears, external payment arrears will accrue when undisputed payments such as interest or amortization on debts of the government (as defined in paragraph 7) to non-residents are not made within the terms of the contract.

\section{E. Ceiling on the Contracting or Guaranteeing of New Nonconcessional External Debt}

29. A ceiling applies to the contracting and guaranteeing of new medium-to-long term nonconcessional external debt by the government and the BoG, and the following public enterprises: (i) Tema Oil Refinery; (ii) Ghana National Petroleum Company; (iii) Bulk Oil Storage and Transport Company; (iv) Volta River Authority; and (v) Electricity Company of Ghana. The ceiling applies to debt and commitments contracted or guaranteed for which value has not yet been received.

30. The 2011 ceiling on the contracting or guaranteeing of new nonconcessional external debt (US\$3,400 million) comprises the following two subceilings: (i) a subceiling for the maximum amount of nonconcessional external debt in the oil and gas sector that can be contracted or guaranteed for oil and gas exploration and production projects in Ghana and to acquire equity stakes in companies undertaking oil and gas exploration and production in Ghana (US\$1,250 million); and (ii) a subceiling for the maximum amount of nonconcessional external debt that can be contracted or guaranteed in any sector other than the oil and gas sector (US\$2,150 million). For 2012, the contracting of nonconcessional external debt of US\$810 million is envisaged to be outside the oil and gas sector.

31. Excluded from the ceiling are (i) the use of Fund resources; and (ii) lending from the World Bank, the African Development Bank, and the International Fund for Agricultural Development.

32. For program purposes, a debt is concessional if it includes a grant element of at least 35 percent, calculated as follows: the grant element of a debt is the difference between the net present value (NPV) of debt and its nominal value, expressed as a percentage of the nominal value of the debt. The NPV of debt at the time of its contracting is calculated by discounting the future stream of payments of debt service due on this debt. The discount rates used for this purpose are the currency specific commercial interest reference rates (CIRRs), published by the Organization for Economic Cooperation Development (OECD). For debt with a maturity of at least 15 years, the ten-year-average CIRR will be used to calculate the NPV of debt and, hence, its grant element. For debt with a maturity of less than 15 years, the six-month average CIRR will be used. To both the ten-year and six-month averages, the same margins for differing repayment periods as those used by the OECD would continue to be 
added ( 0.75 percent for repayment periods of less than 15 years, 1 percent for 15 to 19 years, 1.15 percent for 20 to 29 years, and 1.25 percent for 30 years or more). Loans provided by a private entity will not be considered concessional unless accompanied by a grant or grant element provided by a foreign official entity, such as both components constitute an integrated financing package with a combined grant element equal to at least 35 percent.

\section{F. Adjustors to the Program Targets}

33. Program's quantitative targets are subject to the following adjustors:

\section{Overall fiscal deficit of the government}

34. The deficit ceilings for 2011-12 will be adjusted for excesses and shortfalls in loans and grants as defined below, relative to the program assumptions in the table below. The fiscal deficit will be adjusted:

Upward (or downward) for the full amount of any excess (or shortfall) in concessional project loans. Thus, foreign-financed investment projects, which are not under the short-term control of the government, would be unconstrained, varying in line with project loan financing. ${ }^{3}$

Downward by 50 percent of any shortfall in concessional program loans $^{4}$ of GH $\notin 150$ million or less, and downward by the full amount of any shortfall beyond this amount. Thus, for shortfalls of up to GH\&150 million in external loans, the government would have the option of balancing cuts in expenditures with resort to additional domestic financing. The possible resort to additional domestic financing from this adjuster is effectively capped at GHф75 million, limiting potential crowding-out of private sector credit;

(iii) Upward for the full amount of any excess in concessional program loans, where these are used to repay outstanding domestic arrears at a more rapid pace than programmed. ${ }^{5}$

Upward by 50 percent of any shortfall in program grants of GH $\notin 150$ million or less, with no adjustment for any shortfall beyond this amount. As with adjuster (ii), this gives the option of balancing cuts in spending

\footnotetext{
${ }^{3}$ No adjuster is needed for project grants, as shortfalls/excesses in project grants are precisely offset by shortfalls/excesses in foreign-financed capital spending, leaving the fiscal deficit unaffected.

${ }^{4}$ Program grants and loans are also referred to as budget grants and loans.

${ }^{5}$ Adjusters (iv) and (v) ensure that higher than programmed budget support (grants or loans) are used to repay domestic expenditure arrears as a first priority.
} 
with additional resort to domestic financing. The latter is capped, again, at GHф75 million; ${ }^{6}$

(v)

Downward by the full amount of any excess of program grants, less any use of program grants to repay outstanding domestic arrears at a more rapid pace than programmed; and

Budget Financing Assumptions, 2011-12

(GHc millions, cumulative from the start of the calendar year)

\begin{tabular}{|lcccrr|}
\hline & 2011 & 2011 & 2011 & 2012 & 2012 \\
& Jun & Sept & Dec & Mar & Jun \\
\cline { 2 - 6 } Program grants $^{181}$ & 244 & 287 & 48 & 111 \\
Program loans $^{1}$ & 389 & 424 & 427 & 4 & 108 \\
Project loans $^{1}$ & 340 & 412 & 690 & 255 & 557 \\
\hline
\end{tabular}

Notes: ${ }^{1}$ Concessional financing

\section{Net international reserves of the Bank of Ghana}

35. The net international reserve (NIR) floors will be adjusted upward for any excess of budget grants and loans relative to the program baseline (see text table), except where this financing is used to repay outstanding domestic arrears at a more rapid pace than programmed. The NIR floors will be lowered by any shortfall in budget grants and loans relative to the program baseline up to the equivalent of US\$50 million.

\section{Net domestic financing of the government}

36. The ceiling on net domestic financing (NDF) will be adjusted upward by 50 percent of any shortfall in concessional program loans and grants relative to the program (see paragraph 37), up to a maximum adjustment of GHф75 million for shortfalls in each of program loans and grants (and a maximum combined adjustment of GHф150 million). For higher than programmed loans and grants, the ceiling will be adjusted downward by the full amount, except where these loans or grants are used to repay outstanding domestic arrears at a more rapid pace than programmed. The ceiling will also be adjusted upward by the full amount for a reduction in net arrears paid through bond issuance.

\section{F. Provision of Data to the Fund}

37. Data with respect to the variables subject to performance criteria and indicative targets will be provided to Fund staff on a monthly basis with a lag of no more than eight weeks (except for select data for which the reporting lag is explicitly specified in Table 1).

\footnotetext{
${ }^{6}$ The combined scope for additional domestic financing from adjusters (ii) and (iv) is thus GH $\not 150$ million.
} 
The authorities will transmit promptly to Fund staff any data revisions. For any information (and data) that is (are) relevant for assessing performance against program objectives but is (are) not specifically defined in this memorandum, the authorities will consult with Fund staff. 
Table 1. Ghana: Data to be Reported to the IMF

\begin{tabular}{ll}
\hline Item & Periodicity \\
\hline
\end{tabular}

Fiscal data (to be provided by the MOFEP)

Central budget operations for revenues, expenditures and financing, including clearance of arrears.

Divestiture receipts received by the budget (in cedis and foreign exchange, net of divestiture transactions costs).

The stock of domestic payments arrears by sub-category (as defined in para. 9 of the MEFP)

Monthly, within six weeks of the end of each month.

Monthly, within six weeks of the end of each month.

Quarterly, within six weeks of the end of each quarter

Monetary data (to be provided by the BOG)

Net domestic assets and net international reserves of the BOG.

Monthly, within two weeks of the end of each month.

Detailed balance sheet of the monetary authorities.

Monthly, within four weeks of the end of each month.

Monetary survey detailing the consolidated balance sheet of commercial banks using the new BSD2 Report Form.

Summary position of government committed and uncommitted accounts at BOG, and total financing from BOG. Accompanying table showing composition of other receipts and other expenditure.

Composition of banking system and nonbanking system net claims on government.

Monthly, within four weeks of the end of each month.

Debt registry showing structure and holders of domestic government debt, at face value and at discount. Similar table showing holders of treasury bills for open market operations.

Monthly, within four weeks of the end of each month.

Balance of payments (to be provided by the BOG)

Export and import data on value, volume, and unit values, by major categories and other major balance of payments variables.

Quarterly, with a maximum lag of two months.

Foreign exchange cash flow. Monthly, within four weeks of the end of the month.

(continued) 


\section{Table 1. Ghana: Data to be Reported to the IMF (concluded)}

\author{
External debt and foreign assistance data (to be provided by MOFEP) \\ Information on the concessionality of all new external loans contracted by the \\ government or with a government guarantee.
}

For the coming quarter: (i) total debt service due by creditor, (ii) amount of HIPC

Initiative relief on each transaction, and (iii) debt service paid and the transfers to the HIPC Initiative account by creditor for the previous month. Report should cover government and government-guaranteed debt (as defined in this document).

External debt and external debt service incurred by enterprises with government ownership above 50 percent, even if loans have not been explicitly guaranteed by the government.

Short-term liabilities to nonresidents (maturity in one year or less), including overdraft positions and debt owed or guaranteed by the government or the BoG. Data on the BoG short-term liabilities to nonresident commercial banks on accounts 1201 plus 301 plus Crown Agent).

Disbursements of grants and loans by creditor

Other data (to be provided by GSS)

Overall consumer price index.

National accounts by sector of production, in nominal and real terms.

Electricity pricing (to be provided by the Ministry of Energy) Data on the tariff structure and the cost of producing electricity.

Petroleum pricing (to be provided by the Ministry of Energy)

(i) a breakdown of costs, including the ex-refinery price, duties, levies, and margins, for each of the individual petroleum products; and

(ii) the indicative maximum price approved in the bi-weekly review of petroleum pricing for each of the individual petroleum products.

(iii) the commitments to subsidize oil marketing companies in respect of losses incurred due to administrative prices that fall below cost-recovery levels.

(iv) the cumulative unused balance from the petroleum price hedging operations available to subsidize petroleum products.
Quarterly, within four weeks of the end of each quarter.

Quarterly within four weeks of the end of each quarter.

Quarterly, within three weeks of the end of each quarter.

Quarterly, within three weeks of the end of each quarter.

Quarterly, within four weeks of the end of each quarter.

Monthly, within two weeks of the end of each month.

Annual, within three months of the end of each year (switching to quarterly when they become available).

Quarterly, within four weeks of the end of each quarter.

Bi-weekly, within two days of the completion of the pricing review.

See above.

Monthly, within four weeks of the end of each month.

See above. 


\title{
INTERNATIONAL DEVELOPMENT ASSOCIATION \\ INTERNATIONAL MONETARY FUND
}

\section{GHANA}

\section{Joint IMF and World Bank Debt Sustainability Analysis}

\author{
Prepared by the staffs of the \\ International Monetary Fund and the World Bank \\ Approved by Michael Atingi-Ego and Dominique Desruelle (IMF) \\ and Marcelo Giugale and Jeffrey Lewis (World Bank)
}

November 29, 2011

This debt sustainability analysis (DSA) updates the joint IMF/World Bank DSA of May 4, 2011, and reflects the most recent macroeconomic developments, including the authorities' plans to scale up infrastructure investment. ${ }^{1}$ The analysis shows Ghana's external debt burden indicators remaining below their respective indicative thresholds, provided the programmed fiscal consolidation is achieved. However, the risk of external debt distress remains moderate, unchanged from the May DSA. The main vulnerabilities related to a high debt service-to-revenue ratio and continuing risks to the fiscal outlook. Indeed, while overall public sector debt is projected to remain broadly unchanged in relation to GDP, a ratio of 40 percent does not provide strong buffers against shocks, suggesting a case for further gradual consolidation and additional revenue mobilization over the medium term.

\section{ASSUMPTIONS UNDERLYING THE DSA}

\section{Baseline macroeconomic assumptions}

1. This DSA is consistent with the macroeconomic framework outlined in the IMF Staff Report for the Fifth Review under the Extended Credit Facility. Compared to the previous DSA (see Text Table 1 and Box 1), ${ }^{2}$ this update is based on:

- A revised macroeconomic framework. A combination of fiscal consolidation and disinflation has supported the favorable macroeconomic setting in 2011 , and the outlook going forward remains positive. Inflation has stabilized at $81 \frac{1}{2}$ percent, and

\footnotetext{
${ }^{1}$ The DSA presented in this document is based on the standard low-income countries (LIC) DSA framework. See "Debt Sustainability in Low-Income Countries: Further Considerations on an Operational Framework, Policy Implications"http://www.imf.org/external/np/pdr/sustain/2004/091004.htm.

${ }^{2}$ See IMF Country Report No. 11/128 http://www.imf.org/external/pubs/cat/longres.aspx?sk=24912.0
} 
the government is on track to reduce the fiscal deficit (after arrears clearance) by about $2 \frac{1}{4}$ percentage points of (non-oil) GDP. The DSA assumes implementation of agreed policies in 2012 and unchanged policies thereafter, corresponding to a primary fiscal deficit of $1 / 2$ percent of GDP from 2013 onward. Despite significant export growth, the external current account deficit for 2011 is projected to remain broadly unchanged at $8 \frac{1}{4}$ percent of GDP, reflecting a strong rebound in imports, and to gradually improve in the long run.

- A revised public investment profile. The Ghanaian authorities are planning a significant scaling up of infrastructure investment and have negotiated a large financing package of US\$3 billion on nonconcessional terms to finance critical investments. The loan amounts are assumed to be disbursed in equal installments of US\$750 million a year over a four-year period. Beyond this loan, it is assumed that there will be a general shift toward more nonconcessional external borrowing (NCB) than previously projected.

- Revised grant financing projections. Consistent with Ghana's lower middle-income status, it is expected that grant financing will continue to fall over the projection period. 
Text Table 1. Key Macroeconomic Assumptions

\begin{tabular}{|c|c|c|c|}
\hline & 2010 & 2011-16 & 2017-30 \\
\hline Real GDP growth & \multicolumn{3}{|c|}{ (annual percentage change) } \\
\hline DSA - May 2011 & 5.7 & 7.4 & 5.0 \\
\hline DSA - November 2011 & 7.7 & 7.7 & 5.4 \\
\hline Level of nominal GDP & \multicolumn{3}{|c|}{ (in million U.S. dollar, e.o.p.) } \\
\hline DSA - May 2011 & 31,084 & 62,479 & 215,157 \\
\hline DSA - November 2011 & 32,321 & 59,359 & 181,462 \\
\hline Inflation (GDP deflator) & \multicolumn{3}{|c|}{ (annual percentage change) } \\
\hline DSA - May 2011 & 14.1 & 9.1 & 7.2 \\
\hline DSA - November 2011 & 17.3 & 8.9 & 5.9 \\
\hline Non-interest current account balance & \multicolumn{3}{|c|}{ (in percent of GDP) } \\
\hline DSA - May 2011 & -6.6 & -3.5 & -3.0 \\
\hline DSA - November 2011 & -7.6 & -4.7 & -3.6 \\
\hline Primary fiscal balance & \multicolumn{3}{|c|}{ (in percent of non-oil GDP) } \\
\hline DSA - May 2011 & -4.4 & 0.6 & -0.2 \\
\hline DSA - November 2011 & -4.3 & -1.0 & -0.5 \\
\hline
\end{tabular}




\section{Box 1: Baseline Macroeconomic Assumptions}

Real GDP growth: Growth has been strong, rising from 4 percent in 2009 to an estimated $13 \frac{1}{2}$ percent in 2011 , boosted by oil production and robust expansion of the non-oil economy. The overall economy is projected to grow by more than 8 percent in 2012 with a gradual decline to an average of $5 \frac{1}{2}$ percent in the long term. Real growth projections are higher than in the previous DSA, but remain conservative. Despite the scaling up of infrastructure investment and the prospect for new oil and gas discoveries to come on stream, average long-term growth is assumed to stay below the 10-year historical average of 5.8 percent.

Inflation: Inflation has fallen to single digits from its peak of more than 20 percent in June 2009. It is expected to remain broadly stable at around 8-9 percent in 2011 and gradually decline to $5 \frac{1}{2}$ percent in the long term.

Government balances: Based on the strong performance - particularly on the revenue sideduring the first three quarters of the year, the 2011 fiscal deficit target is projected to decline below 5 percent of (non-oil) GDP. After a temporary increase in the deficit in 2012, mainly as a result of one-off retroactive wage payments, it is assumed that the primary deficit will remain unchanged at $1 / 2$ percent of GDP throughout the projection period.

Current account balance: Ghana's external balance continues to benefit from strong cocoa and gold exports. The non-interest current account deficit is projected to gradually narrow from around 8 percent of GDP in 2011 to 3-4 percent at the end of the projection period, broadly in line with the current account norm produced by the macroeconomic balance approach. Exports are projected to grow at a robust pace to close to 40 percent of GDP by 2031, increasingly supported by nontraditional exports as infrastructure bottlenecks are reduced. Imports are also projected to rise to around 46 percent of GDP, with a significant portion devoted to equipment for new oil fields and government infrastructure projects in the near term.

FDI: In the financial account, non-debt creating inflows (largely composed of foreign direct investment) are projected to decline from the current high levels (around 8 percent of GDP) to about 3-4 percent in the long-term, as the initial investments in the oil and gas sector abate. The baseline assumes that oil extraction is limited to the Jubilee 1 field, with oil-related investments projected to decelerate. This is a conservative assumption in light of new discoveries.

Debt financing: Concessional financing from official bilateral creditors is expected to decline, and to be gradually replaced by commercial borrowing over the medium to long term. The shift is consistent with Ghana's recent transition to lower middle-income status. The projected volume of commercial borrowing is higher than in the previous DSA, consistent with Ghana's plans to scale up infrastructure investment. The DSA assumes that the ECF program limits on contracting of nonconcessional external debt will be fully utilized during 2011-12, with annual disbursements of close to 3 percent of GDP during 2012-15 (Text Table 2). Net foreign financing is projected to remain above 2 percent of GDP annually through the end of the projection period, with net domestic financing as a residual averaging 1 1/4 percent of GDP during 2012-31. Ghana's \$750 million sovereign bond is expected to be rolled over in 2017 and 2027. 
Text Table 2. Official Public External Borrowing (in US\$ millions)

\begin{tabular}{|c|c|c|c|c|c|c|c|}
\hline & & & & & & & Average \\
\hline & 2011 & 2012 & 2013 & 2014 & 2015 & 2016 & 2017-31 \\
\hline \multicolumn{8}{|l|}{ November 2011 DSA } \\
\hline Total disbursements & 1,297 & 2,223 & 1,920 & 2,021 & 1,993 & 2,005 & 5,542 \\
\hline Concessional loans & 1,128 & 980 & 657 & 707 & 623 & 623 & 674 \\
\hline Nonconcessional loans & 168 & 1,243 & 1,263 & 1,314 & 1,370 & 1,383 & 4,869 \\
\hline (percent of GDP) & 0.4 & 2.9 & 2.7 & 2.6 & 2.5 & 2.3 & 4.1 \\
\hline \multicolumn{8}{|l|}{ May 2011 DSA } \\
\hline Total disbursements & 2,166 & 1,759 & 1,392 & 1,415 & 1,541 & 1,708 & 3,836 \\
\hline Nonconcessional loans & 648 & 500 & 600 & 700 & 826 & 943 & 3,071 \\
\hline
\end{tabular}

\section{EXTERnAL DSA}

\section{Baseline scenario}

\section{Under the baseline scenario, Ghana's debt indicators are below the relevant} indicative debt distress thresholds. ${ }^{3}$ The projected level and composition of external debt is associated with a small deterioration in the various debt burden indicators, but all remain below their respective thresholds (Text Table 3, Tables 1 and Figure 1). The baseline external debt burden trajectories are less favorable than in the May 2011 DSA, however, reflecting mainly assumptions on increased external borrowing on non-concessional terms.

\section{Standard stress tests}

3. Standard stress tests confirm a moderate risk of debt distress (Table 2). On the positive side, all three stock indicators remain below their threshold levels under the stress scenarios. However, in the worst stress-test scenario (a one-time 30 percent exchange rate depreciation), the external debt-to-GDP ratio fails to stabilize and approaches its indicative threshold by the end of the projection period. Moreover, the external debt service-to-revenue ratio breaches its threshold under the same stress test, rising to 40 percent by 2031. A permanently higher nominal interest rate on new external borrowing also moves debt burden indicators closer to the thresholds and raises the external debt-service ratio significantly

\footnotetext{
${ }^{3}$ As in the previous DSA, public debt is determined net of government deposits at the banking system, including the Oil Fund held at the Bank of Ghana. The difference between gross and net debt is about $2^{1 / 2}$ percent of non-oil GDP. Reflecting a lack of reliable data, nonguaranteed debt of state-owned enterprises is excluded, and private sector external debt data are weak.
} 
above the threshold to almost 50 percent of revenues by the end of the projection period. ${ }^{4}$ Keeping key variables at their historical levels, on the other hand, would imply lower debt indicators in the long run, reflecting the baseline assumption of a considerably lower nominal GDP growth rate (in U.S. dollar terms) than the average observed over the past 10 years. ${ }^{5}$

Text Table 3. Indicators of External Debt Vulnerabilities (Baseline, in percent)

\begin{tabular}{|lrrr|}
\hline & 2016 & 2021 & 2031 \\
\hline PV of debt-to -GDP ratio & & & \\
May 2011 DSA & 19.4 & 21.2 & 22.9 \\
November 2011 DSA & 25.4 & 28.9 & 32.6 \\
Threshold & 50 & 50 & 50 \\
\hline PV of debt-to-exports ratio & & & \\
May 2011 DSA & 56.0 & 67.2 & 72.8 \\
November 2011 DSA & 66.3 & 76.4 & 83.0 \\
Threshold & 200 & 200 & 200 \\
\hline PV of debt-to-revenue ratio & & & \\
May 2011 DSA & 96.6 & 116.5 & 132.4 \\
November 2011 DSA & 124.4 & 154.8 & 182.0 \\
Threshold & 300 & 300 & 300 \\
\hline Debt service-to-exports ratio & & & \\
May 2011 DSA & 3.5 & 6.4 & 8.5 \\
November 2011 DSA & 4.3 & 9.4 & 13.1 \\
Threshold & 25 & 25 & 25 \\
\hline Debt service-to-revenue ratio & & & \\
May 2011 DSA & 6.0 & 11.1 & 15.5 \\
November 2011 DSA & 19.0 & 28.8 \\
Threshold & 35 & 35 & 35 \\
\hline Sources: IMF staff projections. & 35 & \\
1/ All indicatators refer to public and publicly guaranteed external debt. \\
\hline
\end{tabular}

\section{Public Sector DSA}

\section{The public debt dynamics, covering both external and domestic liabilities,} indicate additional risks (Text Table 4). Although public debt, as a share of GDP, is projected to remain broadly stable in the baseline, a ratio of 40 percent is already sizeable

\footnotetext{
${ }^{4}$ The standard stress test assumes that the interest rate on new borrowing is two percentage points higher than in the baseline scenario.

${ }^{5}$ In the historical scenario, the variables that are kept at their 2001-10 levels are output growth, inflation (measured by dollar GDP deflator), and the non-interest current account balance and FDI in percent of GDP.
} 
and could rise significantly in the event of shocks or failure to achieve the anticipated consolidation (Table 3 and Figure 2). Indeed, keeping the primary deficit at its projected 2011 level of $1 \frac{1}{2}$ percent of GDP, rather than reducing it to $1 / 2$ percent of GDP from 2013 onward (as assumed in the baseline), would imply a steady rise in the public debt ratio to about 65 percent of GDP by 2031 (Table 4). The debt ratio would rise to almost 80 percent, if the primary balance and real GDP growth were at their historical averages. Moreover, a high and rising debt service-to-revenue ratio, even in the baseline, suggests the need for further reforms to boost revenues over the medium to long term, building on recent successes and new initiatives planned for 2012 .

Text Table 4. Indicators of Public Debt Vulnerabilities (Baseline, in percent)

\begin{tabular}{|c|c|c|c|}
\hline & 2016 & 2021 & 2031 \\
\hline \multicolumn{4}{|l|}{ PV of debt-to -GDP ratio } \\
\hline May 2011 DSA & 30.5 & 37.9 & 42.1 \\
\hline November 2011 DSA & 34.7 & 36.7 & 41.7 \\
\hline \multicolumn{4}{|c|}{ PV of debt-to-revenue ratio $2 /$} \\
\hline May 2011 DSA & 143.9 & 201.8 & 240.3 \\
\hline November 2011 DSA & 162.6 & 191.4 & 230.9 \\
\hline \multicolumn{4}{|c|}{ Debt service-to-revenue ratio $2 /$} \\
\hline May 2011 DSA & 27.3 & 41.8 & 52.8 \\
\hline November 2011 DSA & 27.3 & 41.1 & 53.1 \\
\hline \multicolumn{4}{|c|}{$\begin{array}{l}\text { Sources: IMF staff projections. } \\
\text { 1/ All indicatators refer to total public and publicly guaranteed debt. }\end{array}$} \\
\hline
\end{tabular}

\section{Conclusions}

\section{The baseline DSA is overall less favorable than in May, but the risks remain}

moderate. The deterioration in debt burden trajectories mainly reflects higher external borrowing assumptions. Nevertheless, the trajectories of all external debt burden indicators remain within their respective thresholds, even under stress. The most pronounced risks arise from a high debt service-to-revenue ratio and a total public debt ratio that provides limited buffers against shocks.

\section{As emphasized in the previous analysis, successful fiscal consolidation and continued robust growth will be essential for achieving the projected debt outcomes.}

The baseline scenario assumes that the program objectives are achieved, with a small primary deficit of about $1 / 2$ percent of GDP in 2013 and beyond, and ongoing robust export performance and economic growth. While the growth projections remain conservative based on historical trends, and there is considerable upside potential, unproductive spending could 
easily derail the debt dynamics onto an unsustainable path. Thus, careful expenditure prioritization toward growth-enhancing investment and ongoing efforts to boost revenues will remain important to keep debt sustainable and create buffers against shocks.

\section{Further improvements in debt management capacity will also be essential,} especially in light of Ghana's increasing access to market financing. While the DSA suggests that Ghana can manage a gradual shift toward nonconcessional borrowing, new debts - and the associated projects - need to be selected and managed carefully, with emphasis on favorable terms and sufficiently long maturity to contain rollover risks.

8. The authorities agree with the broad thrust of the analysis, but consider the projections to be on the conservative side. In their view, the projections understate the potential growth dividends from the planned infrastructure investments, particularly in the gas sector. Incorporating such dividends would imply stronger debt indicators. 
Table 1: External Debt Sustainability Framework, Baseline Scenario, 2008-31 1/

(In percent of GDP, unless otherwise indicated)

\begin{tabular}{|c|c|c|c|c|c|c|c|c|c|c|c|c|c|c|c|}
\hline & \multicolumn{3}{|c|}{ Actual } & \multirow{2}{*}{$\begin{array}{l}\text { Historical } \\
\text { Average }\end{array}$} & \multirow{2}{*}{$\begin{array}{l}\text { Standard } \\
\text { Deviation }\end{array}$} & \multicolumn{6}{|c|}{ Projections } & & \multirow[b]{2}{*}{$\begin{array}{c}2017-2031 \\
\text { Average }\end{array}$} \\
\hline & 2008 & 2009 & 2010 & & & 2011 & 2012 & 2013 & 2014 & 2015 & 2016 & $\begin{array}{c}2011-2016 \\
\text { Average }\end{array}$ & 2021 & 2031 & \\
\hline External debt (nominal) 1/ & 24.0 & 27.6 & 27.5 & 48.1 & 28.9 & 26.1 & 27.7 & 30.0 & 32.0 & 33.2 & 34.6 & 30.6 & 37.9 & 40.8 & 38.7 \\
\hline $\mathrm{o} / \mathrm{w}$ public and publicly guaranteed (PPG) & 16.2 & 19.5 & 19.9 & 38.5 & 26.8 & 19.7 & 22.2 & 24.1 & 25.6 & 26.4 & 27.3 & 24.2 & 28.7 & 29.5 & 28.9 \\
\hline Change in external debt & 0.2 & 3.7 & -0.1 & -9.5 & 12.6 & -1.4 & 1.5 & 2.4 & 2.0 & 1.2 & 1.3 & 1.2 & 0.4 & 0.2 & 0.4 \\
\hline Identified net debt-creating flows & 3.4 & 0.1 & -5.2 & -5.6 & 8.4 & -3.1 & -3.3 & -1.7 & -0.7 & -0.7 & -0.5 & -1.7 & -0.7 & 0.4 & -0.4 \\
\hline Non-interest current account deficit & 10.2 & 3.4 & 7.6 & 4.3 & 3.5 & 7.9 & 5.6 & 3.2 & 3.9 & 4.0 & 3.6 & 4.7 & 3.6 & 3.6 & 3.6 \\
\hline Deficit in balance of goods and services & 19.3 & 13.1 & 13.8 & 13.8 & 3.3 & 12.1 & 9.8 & 6.9 & 7.8 & 8.1 & 7.9 & 8.8 & 7.9 & 7.4 & 7.9 \\
\hline Exports & 24.8 & 29.5 & 29.3 & 26.7 & 3.1 & 38.6 & 38.3 & 39.1 & 38.4 & 38.1 & 38.4 & 38.4 & 37.9 & 39.2 & 38.0 \\
\hline Imports & 44.0 & 42.6 & 43.1 & 40.4 & 3.6 & 50.7 & 48.0 & 46.0 & 46.2 & 46.1 & 46.3 & 47.2 & 45.8 & 46.6 & 45.9 \\
\hline Net current transfers (negative $=$ inflow) & -9.4 & -10.2 & -7.2 & -10.0 & 1.4 & -6.3 & -6.9 & -6.3 & -6.3 & -6.3 & -6.3 & -6.4 & -5.4 & -3.9 & -5.0 \\
\hline $\mathrm{o} / \mathrm{w}$ official & -2.5 & -3.3 & -0.6 & -2.4 & 0.9 & -1.3 & -1.3 & -0.9 & -0.9 & -0.8 & -0.6 & -0.9 & -0.3 & -0.1 & -0.2 \\
\hline Other current account flows (negative $=$ net inflow) & 0.3 & 0.6 & 1.1 & 0.5 & 0.3 & 2.1 & 2.8 & 2.6 & 2.4 & 2.2 & 2.0 & 2.3 & 1.1 & 0.1 & 0.7 \\
\hline Net FDI (negative = inflow) & -4.2 & -6.5 & -7.8 & -2.9 & 2.7 & -8.2 & -7.3 & -3.6 & -3.6 & -3.6 & -3.7 & -5.0 & -3.8 & -2.9 & -3.6 \\
\hline Endogenous debt dynamics 2 / & -2.5 & 3.2 & -5.0 & -7.0 & 7.5 & -2.7 & -1.5 & -1.3 & -0.9 & -1.0 & -0.5 & -1.3 & -0.5 & -0.3 & -0.4 \\
\hline Contribution from nominal interest rate & 0.6 & 0.6 & 0.6 & 0.8 & 0.4 & 0.5 & 0.4 & 0.6 & 0.7 & 0.8 & 0.9 & 0.6 & 1.3 & 1.9 & 1.5 \\
\hline Contribution from real GDP growth & -1.7 & -1.1 & -1.7 & -2.6 & 1.4 & -3.2 & -1.9 & -1.9 & -1.7 & -1.8 & -1.4 & -2.0 & -1.8 & -2.2 & -1.9 \\
\hline Contribution from price and exchange rate changes & -1.4 & 3.6 & -3.9 & -5.2 & 7.2 & & & $\ldots$ & & & $\ldots$ & $\ldots$ & 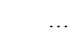 & & \\
\hline Residual (3-4) 3/ & -3.3 & 3.6 & 5.1 & -3.9 & 12.1 & 1.7 & 4.8 & 4.0 & 2.7 & 1.9 & 1.9 & 2.8 & 1.1 & -0.2 & 0.9 \\
\hline $\mathrm{o} / \mathrm{w}$ exceptional financing & -0.2 & -3.9 & 0.0 & -3.6 & 5.0 & -0.1 & -0.1 & -0.1 & 0.0 & 0.0 & 0.0 & -0.1 & 0.0 & 0.0 & 0.0 \\
\hline PV of external debt $4 /$ & $\ldots$ & $\ldots$ & 23.2 & 23.2 & $\ldots$ & 21.9 & 24.1 & 27.0 & 29.4 & 31.0 & 32.7 & 27.7 & 38.1 & 43.9 & 39.9 \\
\hline In percent of exports & $\ldots$ & $\ldots$ & 79.0 & 79.0 & $\ldots$ & 56.8 & 62.9 & 69.0 & 76.6 & 81.5 & 85.4 & 72.0 & 100.7 & 112.0 & 104.8 \\
\hline PV of PPG external debt & $\ldots$ & $\ldots$ & 15.6 & 15.6 & $\ldots$ & 15.5 & 18.6 & 21.0 & 23.0 & 24.2 & 25.4 & 21.3 & 28.9 & 32.6 & 30.0 \\
\hline In percent of exports & $\ldots$ & $\ldots$ & 53.2 & 53.2 & $\ldots$ & 40.2 & 48.5 & 53.7 & 59.8 & 63.5 & 66.3 & 55.3 & 76.4 & 83.0 & 78.9 \\
\hline In percent of government revenues & $\ldots$ & $\ldots$ & 108.3 & 108.3 & $\ldots$ & 85.6 & 99.8 & 112.3 & 115.5 & 119.0 & 124.4 & 109.4 & 154.8 & 182.0 & 162.8 \\
\hline Debt service-to-exports ratio (in percent) & 12.2 & 11.1 & 8.8 & 29.5 & 20.1 & 6.1 & 5.5 & 5.5 & 6.4 & 7.1 & 7.7 & 6.4 & 13.8 & 18.4 & 15.3 \\
\hline PPG debt service-to-exports ratio (in percent) & 6.0 & 5.3 & 4.4 & 22.6 & 19.9 & 2.7 & 2.6 & 2.9 & 3.5 & 4.0 & 4.3 & 3.3 & 9.4 & 13.1 & 10.6 \\
\hline PPG debt service-to-revenue ratio (in percent) & 11.2 & 11.6 & 8.9 & 44.8 & 37.7 & 5.8 & 5.3 & 6.0 & 6.7 & 7.5 & 8.1 & 6.6 & 19.0 & 28.8 & 22.0 \\
\hline Total gross financing need (Millions of U.S. dollars) & 2561 & 48 & 774 & 1883 & 1453 & 754 & 177 & 832 & 1363 & 1671 & 1718 & 1086 & 4205 & 14410 & 6981 \\
\hline Non-interest current account deficit that stabilizes debt ratio & 10.1 & -0.3 & 7.7 & 13.8 & 11.5 & 9.3 & 4.1 & 0.9 & 1.9 & 2.7 & 2.3 & 3.5 & 3.1 & 3.4 & 3.2 \\
\hline \multicolumn{16}{|l|}{ Key macroeconomic ass umptions } \\
\hline Real GDP growth (in percent) & 8.4 & 4.0 & 7.7 & 5.8 & 1.4 & 13.6 & 8.5 & 7.4 & 6.0 & 6.2 & 4.6 & 7.7 & 5.3 & 5.8 & 5.4 \\
\hline GDP deflator in US dollar terms (change in percent) & 6.3 & -13.0 & 16.3 & 10.1 & 11.1 & 3.2 & 5.6 & 0.8 & 2.3 & 2.5 & 2.5 & 2.8 & 2.5 & 2.0 & 2.2 \\
\hline Effective interest rate (percent) $5 /$ & 2.9 & 2.4 & 2.6 & 1.9 & 0.6 & 2.0 & 1.7 & 2.2 & 2.6 & 2.8 & 3.0 & 2.4 & 3.9 & 5.0 & 4.2 \\
\hline Growth of exports of G\&S (US dollar terms, in percent) & 17.8 & 7.6 & 24.6 & 14.9 & 9.1 & 54.1 & 13.7 & 10.6 & 6.4 & 7.9 & 8.0 & 16.8 & 8.1 & 8.9 & 7.9 \\
\hline Growth of imports of G\&S (US dollar terms, in percent) & 24.9 & -12.6 & 26.7 & 16.2 & 14.7 & 37.9 & 8.6 & 3.8 & 8.7 & 8.7 & 7.5 & 12.5 & 7.4 & 8.6 & 7.8 \\
\hline Grant element of new public sector borrowing (in percent) & $\ldots$ & $\ldots$ & $\ldots$ & $\ldots$ & $\ldots$ & 20.8 & 0.1 & -3.3 & -3.4 & -4.5 & -8.0 & 0.3 & -15.9 & -19.0 & -16.2 \\
\hline Government revenues (excluding grants, in percent of GDP) & 13.3 & 13.5 & 14.4 & 13.4 & 0.8 & 18.1 & 18.6 & 18.7 & 19.9 & 20.3 & 20.5 & 19.3 & 18.7 & 17.9 & 18. \\
\hline Aid flows (in Millions of US dollars) $7 /$ & 776 & 776 & 755 & 590 & 243 & 1042 & 867 & 707 & 739 & 727 & 655 & 789 & 411 & 319 & 405 \\
\hline $\mathrm{o} / \mathrm{w}$ Grants & 776 & 776 & 755 & 590 & 243 & 655 & 721 & 561 & 593 & 582 & 509 & 603 & 411 & 319 & 370 \\
\hline $\mathrm{o} / \mathrm{w}$ Concessional loans & 0.3 & 0.2 & 0.2 & 0.2 & 0.1 & 387 & 146 & 146 & 146 & 146 & 146 & 186 & 0 & 0 & 38 \\
\hline Grant-equivalent financing (in percent of GDP) $8 /$ & $\ldots$ & $\ldots$ & $\ldots$ & $\ldots$ & $\ldots$ & 2.5 & 1.7 & 1.1 & 1.0 & 0.9 & 0.6 & 1.3 & -0.2 & -0.9 & -0.4 \\
\hline Grant-equivalent financing (in percent of external financing) $8 /$ & $\ldots$ & $\ldots$ & $\ldots$ & $\ldots$ & $\ldots$ & 47.0 & 24.6 & 20.1 & 20.1 & 19.1 & 13.8 & 24.1 & -4.4 & -15.3 & 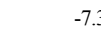 \\
\hline \multicolumn{16}{|l|}{ Memorandum items: } \\
\hline Nominal GDP (Millions of US dollars) & 28,528 & 25,799 & 32,321 & 19,188 & 8,544 & 37,879 & 43,396 & 46,994 & 50,922 & 55,384 & 59,359 & 48,989 & 84,882 & 181,462 & 112,62 \\
\hline Nominal dollar GDP growth & 15.2 & -9.6 & 25.3 & 16.6 & 12.2 & 17.2 & 14.6 & 8.3 & 8.4 & 8.8 & 7.2 & 10.7 & 7.9 & 7.9 & 7.7 \\
\hline PV of PPG external debt (in Millions of US dollars) & $\ldots$ & & 4,894 & & & 5,771 & 7,791 & 9,563 & 11,353 & 13,046 & 14,749 & 10,379 & 24,127 & 58,083 & 33,866 \\
\hline
\end{tabular}

Sources: Ghanaian authorities; and staffestimates and projections.

1/ Includes both public and private sector external debt.

2/ Derived as $[\mathrm{r}-\mathrm{g}-\rho(1+\mathrm{g})](1+\mathrm{g}+\rho+\mathrm{g} \rho)$ times previous period debt ratio, with $\mathrm{r}=$ nominal interest rate; $\mathrm{g}=$ real GDP growth rate, and $\rho=$ growth rate of GDP deflator in U.S. dollar terms.

3/ Includes exceptional financing (i.e., changes in arrears and debt relief); changes in gross foreign assets; and valuation adjustments. For projections also includes contribution from price and exchange rate changes.

4/ Assumes that PV of private sector debt is equivalent to its face value.

5/ Current-year interest payments divided by previous period debt stock.

6/ Historical averages and standard deviations are generally derived over the past 10 years, subject to data availability.

7/ Defined as grants, concessional loans, and debt relief.

8/ Grant-equivalent financing includes grants provided directly to the government and through new borrowing (difference between the face value and the PV of new debt). 
Figure 1: Indicators of Public and Publicly Guaranteed External Debt under Alternative Scenarios, 2011-31 1/

a. Debt Accumulation

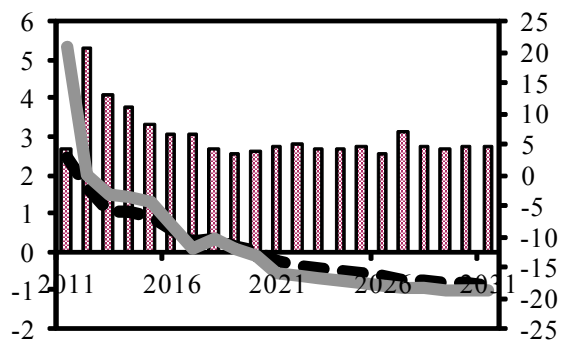

Rate of Debt Accumulation

- -0 Grant-equivalent financing (\% of GDP)

Grant element of new borrowing (\% right scale)

c.PV of debt-to-exports ratio

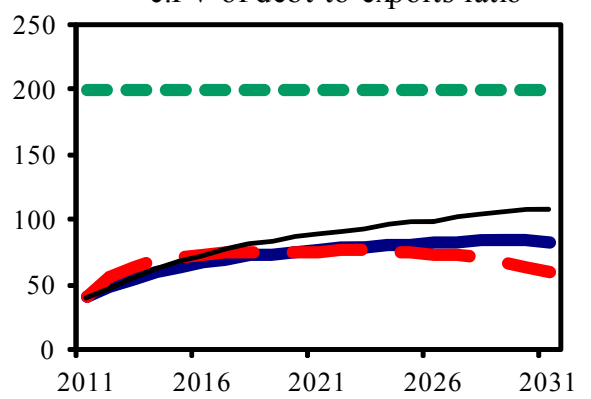

e.Debt service-to-exports ratio

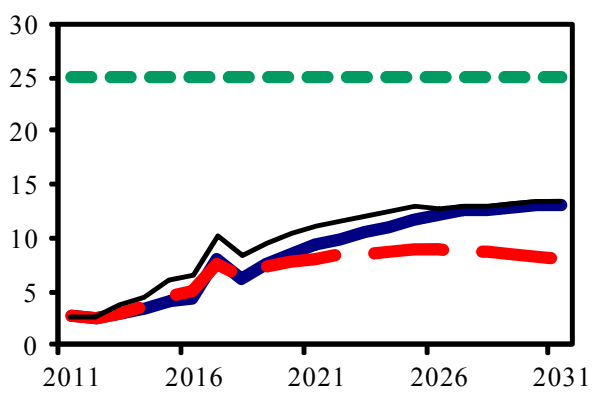

b.PV of debt-to GDP ratio

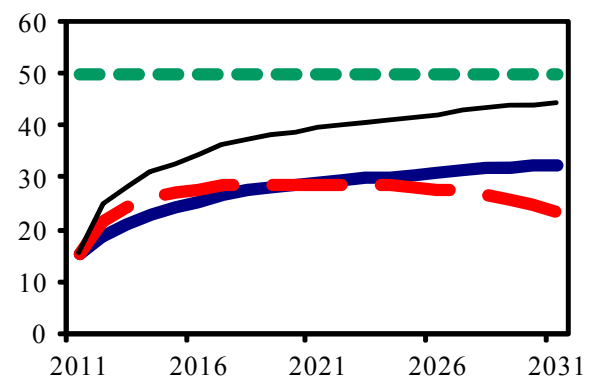

d.PV of debt-to-revenue ratio

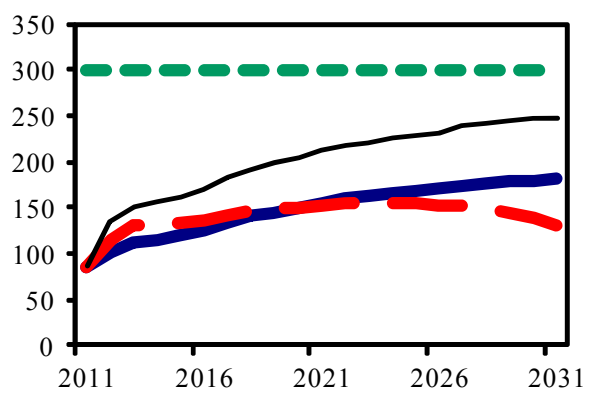

$\longrightarrow$ Baseline $\rightleftharpoons$ Historical scenario Most extreme shock $-\infty$ Threshold

Sources: Ghanaian authorities; and staff estimates and projections.

1/ The most extreme stress test is the test that yields the highest ratio in 2021. 
Table 2. Ghana: Sensitivity Analysis for Key Indicators of Public and Publicly Guaranteed External Debt, 2011-2031

(In percent)

\begin{tabular}{|c|c|c|c|c|c|c|c|c|}
\hline & \multicolumn{8}{|c|}{ Projections } \\
\hline & 2011 & 2012 & 2013 & 2014 & 2015 & 2016 & 2021 & 2031 \\
\hline \multicolumn{9}{|c|}{ PV of de bt-to GDP ratio } \\
\hline Baseline & 15.5 & 18.6 & 21.0 & 23.0 & 24.2 & 25.4 & 28.9 & 32.6 \\
\hline \multicolumn{9}{|l|}{ A. Alternative Scenarios } \\
\hline A1. Key variables at their historical averages in 2011-2031 1/ & 15.5 & 21.4 & 24.4 & 26.1 & 26.9 & 27.8 & 28.5 & 23.4 \\
\hline A2. New public sector loans on less favorable terms in 2011-2031 2 & 15.5 & 18.2 & 21.2 & 23.9 & 25.7 & 27.5 & 33.5 & 42.6 \\
\hline \multicolumn{9}{|l|}{ B. Bound Tests } \\
\hline B1. Real GDP growth at historical average minus one standard deviation in 2012-2013 & 15.5 & 17.9 & 20.9 & 23.2 & 24.5 & 25.9 & 29.5 & 33.2 \\
\hline B2. Export value growth at historical average minus one standard deviation in 2012-2013 3/ & 15.5 & 17.9 & 22.3 & 24.2 & 25.4 & 26.5 & 29.2 & 31.9 \\
\hline B3. US dollar GDP deflator at historical average minus one standard deviation in 2012-2013 & 15.5 & 19.1 & 22.1 & 24.2 & 25.5 & 26.9 & 30.7 & 34.5 \\
\hline B4. Net non-debt creating flows at historical average minus one standard deviation in 2012-2013 4/ & 15.5 & 24.5 & 28.0 & 29.7 & 30.3 & 30.9 & 31.6 & 32.3 \\
\hline B5. Combination of B1-B4 using one-half standard deviation shocks & 15.5 & 22.4 & 23.3 & 25.4 & 26.2 & 27.3 & 29.6 & 32.1 \\
\hline B6. One-time 30 percent nominal depreciation relative to the baseline in 2012 5/ & 15.5 & 25.1 & 28.4 & 31.1 & 32.8 & 34.6 & 39.5 & 44.4 \\
\hline
\end{tabular}

PV of debt-to-exports ratio

Baseline

A. Alternative Scenarios

A1. Key variables at their historical averages in 2011-2031 1/

A2. New public sector loans on less favorable terms in 2011-2031 2

B. Bound Tests

B1. Real GDP growth at historical average minus one standard deviation in 2012-2013

B2. Export value growth at historical average minus one standard deviation in 2012-2013 3/

B3. US dollar GDP deflator at historical average minus one standard deviation in 2012-2013

B4. Net non-debt creating flows at historical average minus one standard deviation in 2012-2013 4/

B5. Combination of B1-B4 using one-half standard deviation shocks

B6. One-time 30 percent nominal depreciation relative to the baseline in 20125 /

$\begin{array}{rrrrrrrr}40.2 & 48.5 & 53.7 & 59.8 & 63.5 & 66.3 & \mathbf{7 6 . 4} & 83.0 \\ & & & & & & & \\ 40.2 & 55.8 & 62.4 & 68.1 & 70.8 & 72.4 & \mathbf{7 5 . 2} & 59.7 \\ 40.2 & 47.7 & 54.3 & 62.3 & 67.6 & 71.6 & \mathbf{8 8 . 5} & 108.7\end{array}$

$\begin{array}{llllllll}40.2 & 46.9 & 52.0 & 58.0 & 61.7 & 64.6 & \mathbf{7 4 . 7} & 81.1 \\ 40.2 & 46.9 & 59.8 & 66.4 & 70.1 & 72.6 & \mathbf{8 1 . 3} & 85.7 \\ 40.2 & 46.9 & 52.0 & 58.0 & 61.7 & 64.6 & \mathbf{7 4 . 7} & 81.1 \\ 40.2 & 63.9 & 71.6 & 77.4 & 79.5 & 80.6 & \mathbf{8 3 . 4} & 82.3 \\ 40.2 & 58.0 & 60.2 & 63.7 & 66.5 & 68.5 & \mathbf{7 5 . 4} & 78.9 \\ 40.2 & 46.9 & 52.0 & 58.0 & 61.7 & 64.6 & \mathbf{7 4 . 7} & 81.1\end{array}$

PV of debt-to-revenue ratio

Baseline

99.8

112.3

115.5

119.0

124.4

154.8

1820

A. Alternative Scenarios

A1. Key variables at their historical averages in 2011-2031 1/ A2. New public sector loans on less favorable terms in 2011-20312

$\begin{array}{rrrrrrrr}85.6 & 114.9 & 130.4 & 131.4 & 132.6 & 135.8 & \mathbf{1 5 2 . 5} & 130.8 \\ 85.6 & 98.1 & 113.5 & 120.2 & 126.5 & 134.3 & \mathbf{1 7 9 . 4} & 238.2 \\ & & & & & & & \\ & & & & & & & \\ 85.6 & 96.5 & 111.9 & 116.9 & 120.7 & 126.4 & \mathbf{1 5 8 . 1} & 185.6 \\ 85.6 & 96.5 & 119.5 & 121.7 & 124.8 & 129.5 & \mathbf{1 5 6 . 6} & 178.5 \\ 85.6 & 102.9 & 118.2 & 121.6 & 125.6 & 131.5 & \mathbf{1 6 4 . 5} & 193.1 \\ 85.6 & 131.5 & 149.8 & 149.3 & 148.9 & 151.2 & \mathbf{1 6 9 . 0} & 180.4 \\ 85.6 & 120.4 & 124.9 & 127.6 & 129.1 & 133.3 & \mathbf{1 5 8 . 6} & 179.3 \\ 85.6 & 134.8 & 151.9 & 156.4 & 161.4 & 169.1 & \mathbf{2 1 1 . 6} & 248.3\end{array}$

B1. Real GDP growth at historical average minus one standard deviation in 2012-2013 B2. Export value growth at historical average minus one standard deviation in 2012-2013 3/

B3. US dollar GDP deflator at historical average minus one standard deviation in 2012-2013 B4. Net non-debt creating flows at historical average minus one standard deviation in 2012-2013 4/

B5. Combination of B1-B4 using one-half standard deviation shocks

B6. One-time 30 percent nominal depreciation relative to the baseline in 20125 / 
Table 2. Ghana: Sensitivity Analysis for Key Indicators of Public and Publicly Guaranteed External Debt, 2011-2031 (continued) (In percent)

\begin{tabular}{|c|c|c|c|c|c|c|c|c|}
\hline & \multicolumn{8}{|c|}{ Projections } \\
\hline & 2011 & 2012 & 2013 & 2014 & 2015 & 2016 & 2021 & 2031 \\
\hline \multicolumn{9}{|c|}{ Debt service-to-exports ratio } \\
\hline Baseline & 2.7 & 2.6 & 2.9 & 3.5 & 4.0 & 4.3 & 9.4 & 13.1 \\
\hline \multicolumn{9}{|l|}{ A. Alternative Scenarios } \\
\hline A1. Key variables at their historical averages in 2011-2031 1/ & 2.7 & 2.6 & 3.1 & 3.7 & 4.6 & 5.0 & 8.1 & 7.9 \\
\hline A2. New public sector loans on less favorable terms in 2011-2031 2 & 2.7 & 2.6 & 2.6 & 3.1 & 3.5 & 3.8 & 10.0 & 21.8 \\
\hline \multicolumn{9}{|l|}{ B. Bound Tests } \\
\hline B1. Real GDP growth at historical average minus one standard deviation in 2012-2013 & 2.7 & 2.6 & 2.9 & 3.5 & 4.0 & 4.3 & 9.3 & 13.1 \\
\hline B2. Export value growth at historical average minus one standard deviation in 2012-2013 3/ & 2.7 & 2.6 & 3.0 & 4.0 & 4.5 & 5.2 & 10.3 & 13.8 \\
\hline B3. US dollar GDP deflator at historical average minus one standard deviation in 2012-2013 & 2.7 & 2.6 & 2.9 & 3.5 & 4.0 & 4.3 & 9.3 & 13.1 \\
\hline B4. Net non-debt creating flows at historical average minus one standard deviation in 2012-2013 4/ & 2.7 & 2.6 & 3.7 & 4.5 & 6.1 & 6.5 & 11.1 & 13.4 \\
\hline B5. Combination of B1-B4 using one-half standard deviation shocks & 2.7 & 2.6 & 3.4 & 3.8 & 4.9 & 5.0 & 9.7 & 12.7 \\
\hline B6. One-time 30 percent nominal depreciation relative to the baseline in $20125 /$ & 2.7 & 2.6 & 2.9 & 3.5 & 4.0 & 4.3 & 9.3 & 13.1 \\
\hline
\end{tabular}

Debt service-to-revenue ratio

Baseline

$$
5.8
$$$$
5.3
$$

6.0

6.7

7.5

8.1

$19.0 \quad 28.8$

\section{A. Alternative Scenarios}

A1. Key variables at their historical averages in 2011-2031 1/ A2. New public sector loans on less favorable terms in 2011-2031 2

$\begin{array}{llllllll}5.8 & 5.3 & 6.4 & 7.1 & 8.6 & 9.3 & \mathbf{1 6 . 5} & 17.4 \\ 5.8 & 5.3 & 5.4 & 5.9 & 6.6 & 7.1 & \mathbf{2 0 . 4} & 47.7\end{array}$

\section{B. Bound Test}

B1. Real GDP growth at historical average minus one standard deviation in 2012-2013

B2. Export value growth at historical average minus one standard deviation in 2012-2013 3/

B3. US dollar GDP deflator at historical average minus one standard deviation in 2012-2013

B4. Net non-debt creating flows at historical average minus one standard deviation in 2012-2013 4/

B5. Combination of B1-B4 using one-half standard deviation shocks

B6. One-time 30 percent nominal depreciation relative to the baseline in 20125 /

$\begin{array}{rrrrrrrr}5.8 & 5.3 & 6.1 & 7.0 & 7.8 & 8.4 & \mathbf{1 9 . 8} & 29.9 \\ 5.8 & 5.3 & 6.0 & 7.2 & 8.0 & 9.2 & \mathbf{1 9 . 9} & 28.8 \\ 5.8 & 5.7 & 6.5 & 7.3 & 8.1 & 8.8 & \mathbf{2 0 . 6} & 31.1 \\ 5.8 & 5.3 & 7.8 & 8.8 & 11.4 & 12.2 & \mathbf{2 2 . 5} & 29.3 \\ 5.8 & 5.4 & 7.1 & 7.6 & 9.6 & 9.8 & \mathbf{2 0 . 4} & 29.0 \\ 5.8 & 7.5 & 8.3 & 9.4 & 10.5 & 11.3 & \mathbf{2 6 . 5} & 40.0\end{array}$

Memorandum item:

Grant element assumed on residual financing (i.e., financing required above baseline) 6/

Sources: Ghanaian authorities; and staff estimates and projections.

1/ Variables include real GDP growth, growth of GDP deflator (in U.S. dollar terms), non-interest current account in percent of GDP, and non-debt creating flows.

2/ Assumes that the interest rate on new borrowing is by 2 percentage points higher than in the baseline., while grace and maturity periods are the same as in the baseline.

3/ Exports values are assumed to remain permanently at the lower level, but the current account as a share of GDP is assumed to return to its baseline level after the shock (implicitly assuming an offsetting adjustment in import levels).

4/ Includes official and private transfers and FDI.

5/ Depreciation is defined as percentage decline in dollar/local currency rate, such that it never exceeds 100 percent.

6/ Applies to all stress scenarios except for A2 (less favorable financing) in which the terms on all new financing are as specified in footnote 2. 
Table 3: Public Sector Debt Sustainability Framework, Baseline Scenario, 2008-31 (In percent of GDP, unless otherwise indicated)

\begin{tabular}{|c|c|c|c|c|c|c|c|c|c|c|c|c|c|c|c|}
\hline & \multicolumn{3}{|c|}{ Actual } & \multirow[b]{2}{*}{ Average } & \multirow[b]{2}{*}{$\begin{array}{l}\text { Standard } \\
\text { Deviation }\end{array}$} & \multicolumn{4}{|l|}{ Estimate } & \multicolumn{3}{|c|}{ Projections } & \multirow[b]{2}{*}{2021} & \multirow[b]{2}{*}{2031} & \multirow[b]{2}{*}{$\begin{array}{l}2017-31 \\
\text { Average }\end{array}$} \\
\hline & 2008 & 2009 & 2010 & & & 2011 & 2012 & 2013 & 2014 & 2015 & 2016 & $\begin{array}{l}2011-16 \\
\text { Average }\end{array}$ & & & \\
\hline Public sector debt 1/ & 33.6 & 36.2 & 46.3 & 55.1 & 26.5 & 42.6 & 43.5 & 42.3 & 38.6 & 37.2 & 36.6 & 40.1 & 36.6 & 38.7 & 37.3 \\
\hline $\mathrm{o} / \mathrm{w}$ foreign-currency denominated & 16.2 & 19.5 & 19.9 & 38.5 & 26.8 & 19.7 & 22.2 & 24.1 & 25.6 & 26.4 & 27.3 & 24.2 & 28.7 & 29.5 & 28.9 \\
\hline Change in public sector debt & 2.5 & 2.6 & 10.1 & -7.9 & 12.9 & -3.7 & 0.9 & -1.2 & -3.8 & -1.3 & -0.6 & -1.6 & 0.2 & 0.1 & 0.1 \\
\hline Identified debt-creating flows & 1.6 & 2.0 & 0.9 & -7.5 & 9.0 & -2.8 & -1.9 & -1.3 & -1.3 & -1.4 & -0.8 & -1.6 & -0.4 & 0.0 & -0.3 \\
\hline Primary deficit & 6.2 & 3.0 & 4.3 & 2.2 & 2.1 & 1.8 & 2.1 & 0.5 & 0.5 & 0.5 & 0.5 & 1.0 & 0.5 & 0.5 & 0.5 \\
\hline Revenue and grants & 16.0 & 16.5 & 16.7 & 16.6 & 1.5 & 19.8 & 20.3 & 19.9 & 21.0 & 21.4 & 21.3 & 20.6 & 19.2 & 18.1 & 18.9 \\
\hline of which: grants & 2.7 & 3.0 & 2.3 & 3.2 & 1.0 & 1.7 & 1.7 & 1.2 & 1.2 & 1.1 & 0.9 & 1.3 & 0.5 & 0.2 & 0.4 \\
\hline Primary (noninterest) expenditure & 22.3 & 19.5 & 21.0 & 18.8 & 2.5 & 21.6 & 22.4 & 20.4 & 21.5 & 21.9 & 21.8 & 21.6 & 19.7 & 18.5 & 19.4 \\
\hline Automatic debt dynamics & -2.1 & -0.8 & -4.0 & -9.1 & 7.5 & -5.4 & -3.9 & -1.7 & -1.7 & -1.8 & -1.2 & -2.6 & -0.9 & -0.5 & -0.8 \\
\hline Contribution from interest rate/growth differential & -3.0 & -1.1 & -2.0 & -3.7 & 1.7 & -5.0 & -3.4 & -1.7 & -1.3 & -1.4 & -0.8 & -2.3 & -0.7 & -0.3 & -0.5 \\
\hline of which: contribution from average real interest rate & -0.6 & 0.2 & 0.6 & -0.4 & 0.8 & 0.6 & 0.0 & 1.3 & 1.1 & 0.8 & 0.8 & 0.8 & 1.2 & 1.8 & 1.4 \\
\hline of which: contribution from real GDP growth & -2.4 & -1.3 & -2.6 & -3.2 & 1.3 & -5.6 & -3.3 & -3.0 & -2.4 & -2.2 & -1.6 & -3.0 & -1.8 & -2.1 & -1.9 \\
\hline Contribution from real exchange rate depreciation & 0.9 & 0.3 & -2.0 & -5.4 & 6.0 & -0.4 & -0.5 & 0.0 & -0.3 & -0.4 & -0.3 & -0.3 & $\ldots$ & $\ldots$ & -0.3 \\
\hline Other identified debt-creating flows & -2.6 & -0.2 & 0.7 & -0.6 & 0.9 & 0.8 & -0.2 & -0.1 & -0.1 & -0.1 & -0.1 & 0.0 & 0.0 & 0.0 & 0.0 \\
\hline Privatization receipts (negative) & -2.3 & 0.0 & 1.0 & -0.3 & 0.8 & 1.0 & 0.0 & 0.0 & 0.0 & 0.0 & 0.0 & 0.2 & 0.0 & 0.0 & 0.0 \\
\hline Recognition of implicit or contingent liabilities & 0.0 & 0.0 & 0.0 & 0.0 & 0.0 & 0.0 & 0.0 & 0.0 & 0.0 & 0.0 & 0.0 & 0.0 & 0.0 & 0.0 & 0.0 \\
\hline Debt relief(HIPC and other) & -0.3 & -0.2 & -0.3 & -0.3 & 0.3 & -0.2 & -0.2 & -0.1 & -0.1 & -0.1 & -0.1 & -0.1 & 0.0 & 0.0 & 0.0 \\
\hline Other (specify, e.g. bank recapitalization) & 0.0 & 0.0 & 0.0 & 0.0 & 0.0 & 0.0 & 0.0 & 0.0 & 0.0 & 0.0 & 0.0 & 0.0 & 0.0 & 0.0 & 0.0 \\
\hline Residual, including asset changes & 1.0 & 0.6 & 9.2 & -0.4 & 8.3 & -1.0 & 2.8 & 0.1 & -2.5 & 0.1 & 0.1 & -0.1 & 0.6 & 0.1 & 0.4 \\
\hline \multicolumn{16}{|l|}{ Other Sustainability Indicators } \\
\hline PV of public sector debt & 17.4 & 16.7 & 42.0 & 18.1 & 9.3 & 38.4 & 39.9 & 39.3 & 35.9 & 35.0 & 34.8 & 37.2 & 36.8 & 41.8 & 38.5 \\
\hline $\mathrm{o} / \mathrm{w}$ foreign-currency denominated & 0.0 & 0.0 & 15.6 & 1.7 & 5.2 & 15.5 & 18.6 & 21.0 & 23.0 & 24.2 & 25.4 & 21.3 & 28.9 & 32.6 & 30.0 \\
\hline $\mathrm{o} / \mathrm{w}$ external & $\ldots$ & $\ldots$ & 15.6 & $\ldots$ & $\ldots$ & 15.5 & 18.6 & 21.0 & 23.0 & 24.2 & 25.4 & 21.3 & 28.9 & 32.6 & 30.0 \\
\hline PV of contingent liabilities (not included in public sector debt) & $\ldots$ & $\ldots$ & $\ldots$ & $\ldots$ & $\ldots$ & $\ldots$ & $\ldots$ & $\ldots$ & ... & $\ldots$ & ..." & $\ldots$ & $\ldots$ & ... & . \\
\hline Gross financing need $2 /$ & 11.5 & 9.3 & 10.4 & 12.4 & 4.4 & 13.1 & 12.4 & 11.2 & 10.2 & 10.1 & 10.1 & 11.2 & 12.3 & 13.5 & 12.7 \\
\hline $\mathrm{PV}$ of public sector debt-to-revenue and grants ratio (in percent) & 108.3 & 100.9 & 251.0 & 100.0 & 64.0 & 193.6 & 196.8 & 197.5 & 170.7 & 163.6 & $163.1^{\prime}$ & 180.9 & 192.0 & 231.5 & 204.7 \\
\hline $\mathrm{PV}$ of public sector debt-to-revenue ratio (in percent) & 130.4 & 123.4 & 291.7 & 120.5 & 73.0 & 212.1 & 214.4 & 210.1 & 180.7 & 172.1 & 170.0 & 193.2 & 197.0 & 233.8 & 208.7 \\
\hline $\mathrm{o} / \mathrm{w}$ external $3 /$ & & & 108.3 & 108.3 & & 85.6 & 99.8 & 112.3 & 115.5 & 119.0 & 124.4 & 109.4 & 154.8 & 182.0 & 162.8 \\
\hline Debt service-to-revenue and grants ratio (in percent) 4 / & 13.9 & 16.4 & 16.9 & 43.4 & 30.6 & 30.2 & 27.6 & 30.7 & 27.4 & 27.2 & 27.5 & 28.4 & 41.3 & 53.3 & 45.0 \\
\hline Debt service-to-revenue ratio (in percent) $4 /$ & 16.8 & 20.1 & 19.6 & 53.9 & 38.2 & 33.1 & 30.0 & 32.6 & 29.0 & 28.6 & 28.6 & 30.3 & 42.3 & 53.9 & 45.9 \\
\hline Primary deficit that stabilizes the debt-to-GDP ratio & 3.7 & 0.4 & -5.9 & -0.6 & 4.8 & 5.6 & 1.2 & 1.7 & 4.3 & 1.8 & $1.1^{\mathrm{F}}$ & 2.6 & 0.4 & 0.4 & 0.4 \\
\hline \multicolumn{16}{|l|}{ Key macroeconomic and fiscal assumptions } \\
\hline Real GDP growth (in percent) & 8.4 & 4.0 & 7.7 & 5.8 & 1.4 & 13.6 & 8.5 & 7.4 & 6.0 & 6.2 & 4.6 & 7.7 & 5.3 & 5.8 & 5.4 \\
\hline Average nominal interest rate on forex debt (in percent) & 4.6 & 3.8 & 3.7 & 2.5 & 1.3 & 2.7 & 2.3 & 2.8 & 3.2 & 3.5 & 3.8 & 3.0 & 5.1 & $6.9^{\mathrm{r}}$ & 5.6 \\
\hline Average real interest rate on domestic debt (in percent) & -6.0 & -1.6 & 1.0 & -1.4 & 5.0 & 2.1 & -1.2 & 4.8 & 3.2 & 2.1 & $2.1^{\prime}$ & 2.2 & 2.7 & $3.6^{\prime}$ & 3.1 \\
\hline Real exchange rate depreciation (in percent, + indicates depreciation) & 6.4 & 2.0 & -11.0 & -8.5 & 7.7 & -2.2 & $\ldots$ & & $\ldots$ & & $\ldots$ & & ... & $\ldots$ & \\
\hline Inflation rate (GDP deflator, in percent) & 20.2 & 16.6 & 17.3 & 20.9 & 7.3 & 9.0 & 11.9 & 7.6 & 8.7 & 8.4 & 7.8 & 8.9 & 6.0 & 5.5 & 5.9 \\
\hline Growth of real primary spending (deflated by GDP deflator, in percent) & 0.1 & -0.1 & 0.2 & 0.1 & 0.2 & 0.2 & 0.1 & 0.0 & 0.1 & 0.1 & 0.0 & 0.1 & 0.0 & 0.1 & 0.0 \\
\hline Grant element of new external borrowing (in percent) & & $\ldots$ & $\ldots$ & $\ldots$ & $\ldots$ & 20.8 & 0.1 & -3.3 & -3.4 & -4.5 & -8.0 & 0.3 & -15.9 & -19.0 & \\
\hline
\end{tabular}

Sources: Ghanaian authorities; and staff estimates and projections.

1/ Public sector comprise central government. The concept of net debt is used.
$2 /$ Gross financing need is defined as the primary deficit plus debt service plus the stock of short-term debt at the end of the last period.

3/ Revenues excluding grants.

the sum of interest and amortization of medium and long-term debt.

$5 /$ Historical averages and standard deviations are generally derived over the past 10 years, subject to data availability. 
Figure 2: Indicators of Public Debt Under Alternative Scenarios, 2011-2031 1/
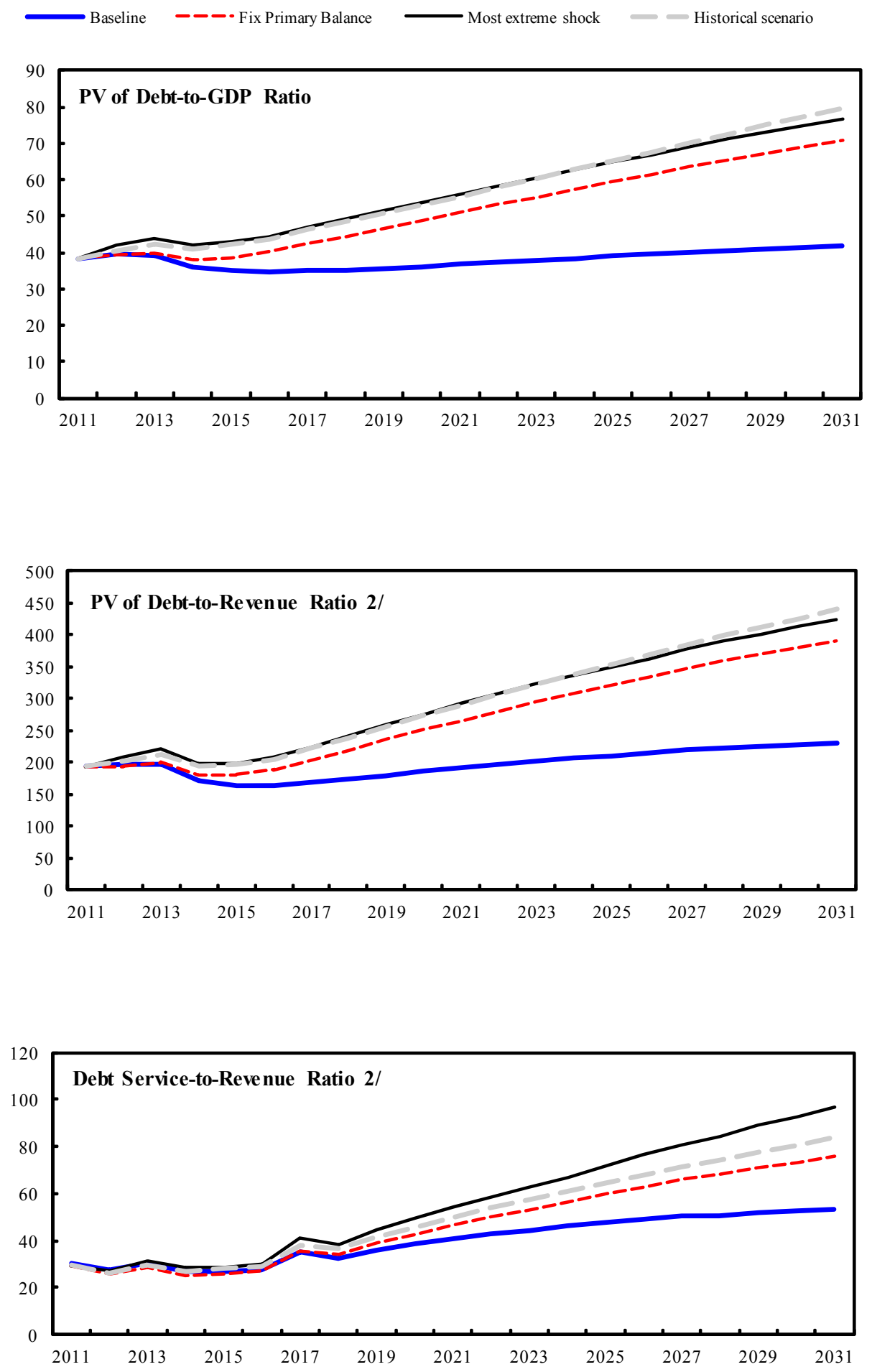

Sources: Ghanaian authorities; and staff estimates and projections.

1/ The most extreme stress test is the test that yields the highest ratio in 2021.

2/ Revenues are defined inclusive of grants. 
INTERNATIONAL MONETARY FUND

GHANA

Fifth Review Under the Three-Year Arrangement Under the Extended Credit Facility and Request for Modification of Performance Criteria-Supplementary Information

Prepared by the African Department

(In consultation with other departments)

Approved by Michael Atingi-Ego and Dominique Desruelle

December 12, 2011

1. The following information has become available to staff since the issuance of the staff report (Fifth Review Under the Three-Year Arrangement Under the Extended Credit Facility and Request for Modification of Performance Criteria).

2. Prior Action on increasing domestic petroleum prices. On December 7, the Ghanaian authorities have informed staff that they will not be able to raise petroleum pump prices before the scheduled Board date, a prior action for the fifth review. The authorities had taken the decision in November to implement the measure before the Board meeting, but implementation has been frustrated by a ruling, on November 28, of the High Court of Ghana. The ruling has ordered the National Petroleum Authority (NPA) to remove a margin introduced in the petroleum pricing formula, which was judged by the court to be illegal. The authorities have mounted an appeal of the ruling, which, in their view is based on a misunderstanding of the petroleum pricing system, and have already filed a motion for stay of execution. In light of the ruling, however, the authorities' concern is that raising petroleum prices now - without having the opportunity to explain their position to the court and the public - could be perceived as frustrating the court's orders. They became aware of these implications only after the staff report was issued.

3. Corrective actions. Since the prior action will not be met, the authorities have taken two corrective actions that demonstrate their ongoing commitment to eliminate petroleum subsidies, as soon as possible: First, the government, via a letter from the Vice President, has informed staff of its commitment to increase petroleum prices to achieve full cost-recovery levels. The letter also spells out that any losses resulting from the delay in the price increase would be covered by holding back domestically-financed capital spending and the reserve expenditure vote (reserve fund). Second, as further evidence of their commitment to implement the price adjustment, the authorities have issued a press release that clarifies the government's position on the court ruling and informs the public of the need to remove costly subsidies and let prices adjust to market conditions. 
4. 2012 Budget. On December 6, Ghana's parliament has approved the budget for 2012, consistent with the agreed program targets.

5. Fiscal Outturn. Preliminary data through October 2011 suggest that revenue proceeds and budget execution are in line with year-end program targets.

6. In view of the prompt corrective actions taken by the authorities demonstrating their continued commitment to increase petroleum prices by the programmed amount and to make any future needed adjustments, the staff recommends completion of the fifth review. 
Table 4.Ghana: Sensitivity Analysis for Key Indicators of Public Debt 2011-2031

\begin{tabular}{|c|c|c|c|c|c|c|c|c|}
\hline & \multicolumn{8}{|c|}{ Projections } \\
\hline & 2011 & 2012 & 2013 & 2014 & 2015 & 2016 & 2021 & 2031 \\
\hline \multicolumn{9}{|l|}{ PV of Debt-to-GDP Ratio } \\
\hline Baseline & 38.4 & 39.9 & 39.3 & 35.9 & 35.0 & 34.8 & 36.8 & 41.8 \\
\hline \multicolumn{9}{|l|}{ A. Alternative scenarios } \\
\hline A1. Real GDP growth and primary balance are at historical averages & 38.4 & 40.9 & 42.6 & 41.0 & 42.3 & 43.9 & 55.6 & 79.5 \\
\hline A2. Primary balance is unchanged from 2011 & 38.4 & 39.5 & 40.2 & 38.2 & 39.1 & 40.7 & 52.1 & 72.8 \\
\hline A3. Permanently lower GDP growth 1 / & 38.4 & 40.0 & 39.5 & 36.3 & 36.0 & 36.6 & 43.4 & 60.4 \\
\hline \multicolumn{9}{|l|}{ B. Bound tests } \\
\hline B1. Real GDP growth is at historical average minus one standard deviations in 2012-2013 & 38.4 & 42.2 & 44.1 & 42.0 & 42.9 & 44.7 & 56.2 & 76.6 \\
\hline B2. Primary balance is at historical average minus one standard deviations in 2012-2013 & 38.4 & 42.3 & 45.9 & 42.5 & 41.8 & 41.9 & 45.5 & 51.2 \\
\hline B3. Combination of B1-B2 using one half standard deviation shocks & 38.4 & 42.3 & 45.6 & 43.2 & 43.7 & 45.2 & 55.1 & 72.4 \\
\hline B4. One-time 30 percent real depreciation in 2012 & 38.4 & 46.8 & 46.1 & 42.9 & 42.6 & 43.3 & 51.9 & 76.4 \\
\hline B5. 10 percent of GDP increase in other debt-creating flows in 2012 & 38.4 & 51.1 & 50.4 & 47.1 & 46.3 & 46.3 & 50.0 & 55.7 \\
\hline
\end{tabular}

PV of Debt-to-Revenue Ratio 2/

Baseline

\section{A. Alternative scenarios}

A1. Real GDP growth and primary balance are at historical averages A2. Primary balance is unchanged from 2011

A3. Permanently lower GDP growth $1 /$

\section{B. Bound tests}

B1. Real GDP growth is at historical average minus one standard deviations in 2012-2013

B2. Primary balance is at historical average minus one standard deviations in 2012-2013

$\mathrm{B} 3$. Combination of B1-B2 using one half standard deviation shocks

B4. One-time 30 percent real depreciation in 2012

B5. 10 percent of GDP increase in other debt-creating flows in 2012 $\begin{array}{llllllll}193.6 & 196.8 & 197.5 & 170.7 & 163.6 & 163.1 & 192.0 & 231.5\end{array}$

$\begin{array}{llllllll}193.6 & 201.2 & 213.7 & 194.3 & 197.5 & 205.8 & 290.3 & 440.4\end{array}$

$\begin{array}{lllllllll}193.6 & 194.8 & 202.1 & 181.7 & 182.8 & 191.2 & 271.8 & 402.9\end{array}$

$\begin{array}{llllllll}193.6 & 197.3 & 198.6 & 172.5 & 168.4 & 171.5 & 226.3 & 334.3\end{array}$

\section{Debt Service-to-Revenue Ratio 2/}

\section{Baseline}

\section{A. Alternative scenarios}

A1. Real GDP growth and primary balance are at historical averages A2. Primary balance is unchanged from 2011

A3. Permanently lower GDP growth $1 /$

$\begin{array}{llllllll}193.6 & 207.6 & 221.1 & 198.9 & 200.0 & 209.0 & 292.9 & 423.8 \\ 193.6 & 208.9 & 231.0 & 202.1 & 195.7 & 196.8 & 237.5 & 283.5 \\ 193.6 & 208.3 & 228.4 & 204.6 & 204.1 & 211.5 & 287.0 & 400.7 \\ 193.6 & 231.0 & 232.1 & 204.0 & 199.3 & 203.2 & 271.1 & 422.7 \\ 193.6 & 252.3 & 253.7 & 223.8 & 216.4 & 217.4 & 261.0 & 308.5\end{array}$

\section{B. Bound tests}

B1. Real GDP growth is at historical average minus one standard deviations in 2012-2013 B2. Primary balance is at historical average minus one standard deviations in 2012-2013 B3. Combination of B1-B2 using one half standard deviation shocks B4. One-time 30 percent real depreciation in 2012

B5. 10 percent of GDP increase in other debt-creating flows in 2012

Sources: Ghanaian authorities; and staff estimates and projections.

1/ Assumes that real GDP growth is at baseline minus one standard deviation divided by the square root of the length of the projection period.

2/ Revenues are defined inclusive of grants. 


\section{INTERNATIONAL MONETARY FUND RETERALAL \\ DEPARTMENT}

Press Release No. 11/469

International Monetary Fund

FOR IMMEDIATE RELEASE

Washington, D.C. 20431 USA

December 14, 2011

\section{IMF Executive Board Completes Fifth Review Under ECF Arrangement for Ghana and Approves US\$91.55 Million Disbursement}

The Executive Board of the International Monetary Fund (IMF) today completed the fifth review of Ghana's economic performance under the program supported by the Extended Credit Facility (ECF). The completion of the review will enable the disbursement of an amount equivalent to SDR 59.58 million (about US\$91.55 million), bringing total disbursements under the arrangement to SDR 268.31 million (about US\$412.28 million).

The Board also approved a modification of a performance criterion related to Ghana's nonconcessional borrowing limit to provide additional room for scaled-up infrastructure investment.

The three-year ECF arrangement was approved on July 15, 2009, with access equivalent to SDR 387.45 million (about US\$595.35 million or 105 percent of quota).

Following the Executive Board's discussion on Ghana, Mr. Naoyuki Shinohara, Deputy Managing Director and Acting Chair, issued the following statement:

"Ghana's economy has improved significantly since the start of the government's Fundsupported program in 2009. The fiscal and external current account imbalances have been greatly reduced, growth has strengthened, inflation has declined to single digits, and international reserves have recovered.

"Pursuit of prudent macroeconomic policies and structural reforms will help preserve the stabilization gains. The government has made great strides in boosting tax revenues and repaying arrears, and has progressed in its structural reform agenda. Remaining fiscal challenges relate to further revenue mobilization, containing current spending, and improving spending efficiency to create space for critical infrastructure investments. The 2012 budget is consistent with making progress in these areas. 
“The government's plans for scaling up critical infrastructure investments translate into significant financing needs. While the debt sustainability analysis suggests scope for higher nonconcessional borrowing, and some of the planned projects promise significant returns, a further strengthening of debt management and project appraisal capacities is critical to keep the debt burden manageable.

"While energy pricing has improved, decisive action is needed to tackle the reemergence of costly and poorly targeted subsidies on petroleum products. Following an initial increase, maintenance of prices at cost-recovery levels will be essential.

"Monetary policy implementation has been consistent with the authorities' inflation target, and the Bank of Ghana should stand ready to adjust policy rates as signs of rising price pressures emerge. To manage liquidity effectively, the Bank of Ghana should continue refining its policies and communication on foreign exchange market interventions.

"Financial sector reforms should focus on making further progress on enhancing supervisory capacity, strengthening banks' risk management, resolving vulnerable institutions and addressing deficiencies in Ghana's Anti-Money Laundering and Combating the Financing of Terrorism (AML/CFT) regime," Mr. Shinohara added. 


\section{Statement by Jafar Mojarrad, Executive Director for Ghana, Mohammed Daïri, Alternative Executive Director, and Alberta Hagan, Advisor December 14, 2011}

Our authorities thank staff for a constructive dialogue during the fifth program review and express their appreciation to Ms. Daseking and her team for their hard work and dedication. The discussions rightly focused on preserving macroeconomic and financial stability while implementing high-priority investments to spur economic growth. Our authorities also thank management and the Board for their continuous support and express appreciation for the high quality Fund TA.

Since approval of the ECF in mid-2009, Ghana has achieved important macroeconomic gains. Growth accelerated to nearly 8 percent in 2010, driven by services, industry, and agriculture, and is projected to surge to $13 \frac{1}{2}$ percent in 2011 and 8 percent in 2012 due to the new oil production and a robust non-oil sector. Inflation has fallen to single digits since June 2010; gross international reserves have risen from the equivalent of less than a month to more than three months of import cover; and the exchange rate has been broadly stable. The banking sector remains adequately capitalized, liquid, and profitable, with declining, even though still high NPLs, and increasing provisions.

Program performance since completion of the third and fourth ECF reviews has been strong. All quantitative performance criteria and indicative targets for end-June were met. Preliminary indications for end-September point to continued strong performance. Structural reforms are also progressing, notwithstanding some delays and deviations from agreed benchmarks, as elaborated in the Memorandum on Economic and Financial Policies. However, the prior action on increase in petroleum prices was not implemented, notwithstanding the authorities' best efforts, as explained in page 3 below.

\section{Recent Macroeconomic and Financial Policies}

Fiscal consolidation, effective monetary policy, a flexible exchange rate policy, and strengthened financial sector oversight have underpinned the favorable macroeconomic outcomes in 2011.

Through a combination of strengthened tax administration and new measures, revenue in 2011 is expected to increase by 5 percent of nonoil GDP and exceed the program projections by 0.8 percentage points, even though oil revenue would be only half of what was projected. Notwithstanding the unexpected higher cost of the transition to single spine pay 
structure $^{1}$ of the public service, which was partly offset through expenditure prioritization, and higher arrears clearance and lower grants, the cash deficit in 2011 is expected to decline by 2.3 percent of nonoil GDP, representing $1 / 2$ percent improvement over program projection. On a commitment basis, the fiscal adjustment in 2011 amounts to an impressive 6 percent of nonoil GDP.

Well-designed monetary policy succeeded in maintaining inflation in single digits, well within the Bank of Ghana's target band. Strengthened confidence and cautious interventions in the foreign exchange market under the floating exchange rate regime have helped reduce volatility and maintain broad stability of the cedi, and the central bank seized the opportunity to strengthen the reserves buffer as needed. Efforts to strengthen risk management in the banking industry continued, and a long-term advisor to help build capacity in banking supervision has been appointed.

\section{Macroeconomic Objectives and Policies for 2012 and the Medium Term}

The authorities' macroeconomic policy agenda has three objectives: preserving the gains in macroeconomic stability and fiscal consolidation; creating space for high-priority investments to spur long-term growth and development; and maintaining single-digit inflation. In the medium term, growth is expected to average 8 percent and reserves to cover four months of imports.

\section{Fiscal Policy}

Fiscal policy will remain prudent while scaling up investment spending to support growth and poverty reduction. Towards this objective, tax revenue would be raised from its relatively low level and recurrent spending contained. Despite lower grants, higher capital spending, and continued arrears clearance, the cash deficit is projected to remain broadly unchanged at just above 5 percent of nonoil GDP in 2012 and to decline to 4 percent in 2013 .

Tax revenue increase would result from new tax policy measures and improvements in administration. New tax measures were announced in the 2012 budget in the areas of natural resources taxation, consistent with Fund TA recommendations, and a review of capital gains taxation will be brought to parliament for approval before end-2012. In addition, the Government is seeking accelerated parliamentary approval of the bill extending VAT to feebased financial services; the bill is expected to be passed in time to be fully implemented in 2012. The authorities also continue to strengthen revenue administration. Senior officials of the GRA have been appointed, the LTO is now fully functional, and pilot integration of VAT and direct taxation under single office heads would begin by end of 2011. The VAT

\footnotetext{
${ }^{1}$ The single-spine salary structure was adopted to help streamline the public sector payroll.
} 
turnover threshold has been increased, consistent with Fund TA advice. Furthermore, tax exemptions are being streamlined, and customs administration strengthened.

The authorities continue to enhance expenditure management and control. Reforms in this area include strengthening of the budget process, upgrading the computerization and infrastructure of expenditure management and control, and strengthening cash management. In addition, migration to the single-spine salary structure is expected to be completed by the end of the year. However, there were delays in mapping employees from the old to the new pay structure because of negotiations and organizational challenges, which resulted in accumulation of sizeable deferred wage payments. The authorities intend to phase the payment of the retroactive wage increases over ten months. Much of the cost overrun would be covered from savings from a payroll audit expected to be completed in the first half of 2012. Progress in strengthening PFM has also been satisfactory. Implementation of the GIFMIS is continuing, even though at a slower pace than expected in view of the complexity of the task, monthly expenditure ceilings have been adopted to facilitate cash management, and transition to a Treasury Single Account is being accelerated.

The authorities remain committed to arrears elimination. They have developed an elaborate database to capture government contractual obligations. On-going arrears prevention measures include monthly monitoring, strengthening planning and expenditure controls, and better cash management. The authorities have substantially reduced arrears and are on track to clear all outstanding arrears by early 2013.

\section{Energy Sector Reforms}

Cost recovery in the energy sector remains a priority. To this end, our authorities remain committed to closely monitor energy prices and adjust prices as needed. Since June 2010, the electricity tariff structure has been reviewed on a quarterly basis, and tariffs are at cost recovery levels. In January 2011, petroleum prices were increased by 30 percent, bringing them to cost-recovery levels. Sizeable hedging gains from the Petroleum Management Hedging Program in the first half of 2011 allowed fuel subsidies to be covered through July. On November 23, 2011, the Cabinet approved a decision to increase domestic petroleum prices by 15 percent, with implementation to be effective December 7, as intended. However, a High Court ruling issued last week required removal of the ex-refinery differential component of the ex-pump prices, effectively preventing the implementation of the increase. The ruling was in a case brought by a local policy and advocacy think tank against the National Petroleum Authority. The Government intends to appeal the ruling, and in the meantime, to take the necessary measures to offset the budgetary impact of any delay in the petroleum price increase. It is also the Government's intention to continue with price adjustments thereafter to ensure full cost-recovery levels. The authorities have consequently requested that the prior action on domestic petroleum price increase be withdrawn, since the 
delay in implementation was due to factors beyond their control and in view of their intention to take corrective actions in the period immediately ahead.

\section{Monetary and Financial Sector Policies}

Building on the increasing credibility of monetary policy under the IT regime, the authorities aim to keep inflation stable in the period ahead with a center-point target of 8.7 percent for 2012. They are also committed to a floating exchange rate regime, with minimal interventions to limit excessive volatility. Going forward, the Bank of Ghana aims to develop a program for foreign exchange market monitoring along with intervention policies to help manage liquidity, drawing on Fund TA.

To strengthen financial stability, on-going priority actions include a review of major financial sector laws in response to the need to strengthen risk-based supervision and crisis prevention and resolution in line with best international practice. In this regard, appropriate regulations to provide for effective implementation of the laws, including resolution powers of the central bank, would be drafted. Furthermore, banks' risk management practices are being enhanced and supervision strengthened, and progress is being made in consolidated and cross-border supervision. Additionally, the BOG is committed to address remaining deficiencies in AML/CFT. To this end, the AML Office created in February this year is being resourced to make it fully operational and is receiving TA from the Fund.

\section{Infrastructure Development and Debt Sustainability}

Ghana faces a large infrastructure deficit, which is a stumbling block to sustained high economic growth, job creation, and poverty alleviation, mainly as a result of dearth of internally-generated and external concessional funding. With its new status as a lower middle-income country and an oil producer, the country's access to concessional funding is projected to decline. To secure financing of vital infrastructure projects in line with the GSGDA, the government has negotiated, and intends to sign by end-2011, a US\$3 billion nonconcessional Master Facility Agreement (MFA) with the Chinese Development Bank.

The World Bank considers the MFA a significant opportunity for Ghana to advance her development agenda. Among the lists of projects, the loan is envisaged to fund a gas infrastructure project, which would utilize a third of the loan amount. The gas project is of utmost importance. As a side product of crude oil production, gas is flared, instead of being harnessed, which represents significant revenue loss and an environmental hazard, in addition to the risks posed to oil fields. The World Bank estimates that returns from the gas project alone would cover 80 percent of the financial cost of the entire MFA amount. The high returns from the gas project would also be used to cross-subsidize some projects with high long term social returns. 
Incorporating this loan, the DSA shows that Ghana's external debt burden indicators remain below all relevant thresholds and the risk of external debt distress moderate-unchanged from the last DSA in May 2011 which did not include the MFA. Continued prudent public debt management and macroeconomic policies, including fiscal consolidation, and careful project selection and implementation would ensure that external and public debts remain sustainable. Implementation of the envisaged projects under the MFA will be phased in yearly tranches of US\$750 million over a four-year period. Our authorities request Fund support in its development efforts by consenting to the requested increase in the ceiling on nonconcessional medium-to-long-term borrowing for December 2011.

\section{Conclusion}

Our authorities are committed to continued strong implementation of their policies and reforms towards preserving macroeconomic and financial stability while seeking to spur long-term growth and poverty reduction. They highly appreciate Fund support in this endeavor, and look forward to the Board's support to their request. 